\title{
Haiti: Fourth Review Under the Three-Year Arrangement Under the Poverty Reduction and Growth Facility, and Request for Waiver of Performance Criterion and Augmentation of Access-Staff Report; Staff Supplement; Press Release on the Executive Board Discussion; and Statement by the Executive Director for Haiti
}

In the context of the fourth review under the three-year arrangement under the Poverty Reduction and Growth Facility, and request for a waiver of performance criterion and augmentation of access, the following documents have been released and are included in this package:

- $\quad$ The staff report for the Fourth Review Under the Three-Year Arrangement Under the Poverty Reduction and Growth Facility, and Request for Waiver of Performance Criterion and Augmentation of Access, prepared by a staff team of the IMF, following discussions that ended on November 14, 2008, with the officials of Haiti on economic developments and policies. Based on information available at the time of these discussions, the staff report was completed on February 2, 2009. The views expressed in the staff report are those of the staff team and do not necessarily reflect the views of the Executive Board of the IMF.

- $\quad$ A staff supplement on the joint IMF/World Bank debt sustainability analysis.

- $\quad$ A Press Release summarizing the views of the Executive Board as expressed during its February 11, 2009 discussion of the staff report that completed the review.

- $\quad$ A statement by the Executive Director for Haiti.

The documents listed below have been or will be separately released.

Letter of Intent sent to the IMF by the authorities of Haiti*

Memorandum of Economic and Financial Policies by the authorities of Haiti*

Technical Memorandum of Understanding*

*Also included in Staff Report

The policy of publication of staff reports and other documents allows for the deletion of market-sensitive information.

Copies of this report are available to the public from

International Monetary Fund $\bullet$ Publication Services

$70019^{\text {th }}$ Street, N.W. $\bullet$ Washington, D.C. 20431

Telephone: (202) 623-7430 • Telefax: (202) 623-7201

E-mail: publications@imf.org・Internet: http://www.imf.org

International Monetary Fund

Washington, D.C. 

INTERNATIONAL MONETARY FUND

HAITI

\section{Fourth Review Under the Three-Year Arrangement Under the Poverty Reduction and Growth Facility, Request for Waiver of Performance Criterion and Augmentation of Access}

Prepared by the Western Hemisphere Department

(In consultation with other departments)

Approved by Gilbert Terrier and Dominique Desruelle

February 2, 2009

\section{PRGF Arrangement}

The IMF Executive Board approved in November 2006 a three-year PRGF arrangement in an amount of 90 percent of quota (SDR 73.71 million), and Haiti's HIPC decision point. The third program review, completed on June 20,2008, included an access augmentation of 20 percent of quota (equivalent to SDR 16.38 million) in response to the food and fuel price shocks. The authorities are requesting a second augmentation of access under the arrangement equivalent to 30 percent of quota (SDR 24.57 million), to mitigate the significant deterioration in the external position caused by a series of hurricanes and flooding in August and September 2008, and the negative impact of the global downturn on remittances and exports of goods and services.

\section{Discussions}

A mission visited Port-au-Prince during November 10-14, 2008 and met with Finance Minister Dorsainvil, Central Bank Governor Castel, other government officials and development partners. The mission consisted of Ms. Redifer (Head), Mr. Di Bella (both WHD), and Messrs. John (PDR) and Bouhga-Hagbe (FAD), and was assisted by Mr. Fasano, Resident Representative.

Mr. Bauer (outgoing Mission Chief) and Ms. Florestal (OED) participated in the policy discussions. Given difficulties in identifying financing for higher spending needs, discussions continued through January from headquarters, led by Ms. Deléchat (Mission Chief). 


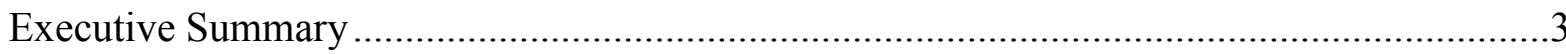

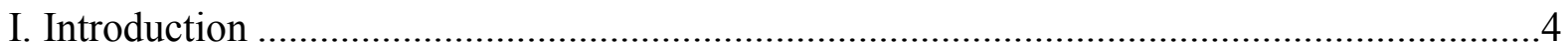

II. Recent Economic Developments ................................................................................

III. Program Performance Through End-September 2008............................................8

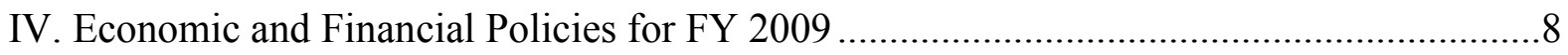

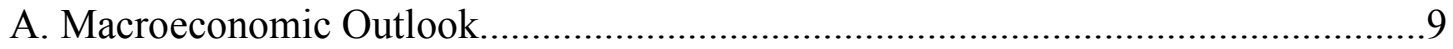

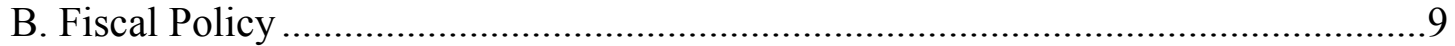

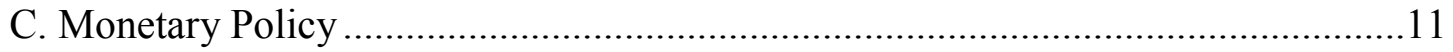

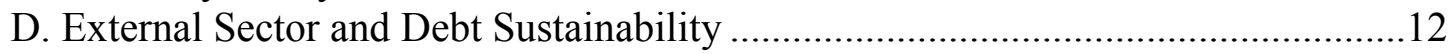

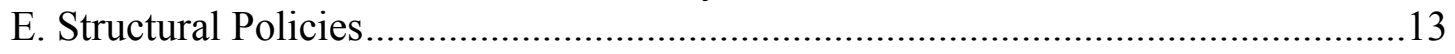

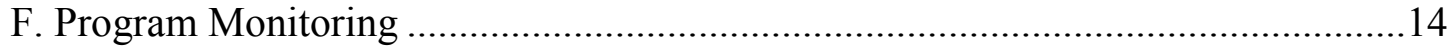

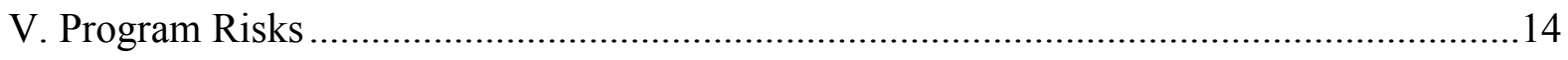

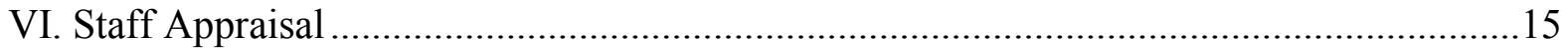

Tables

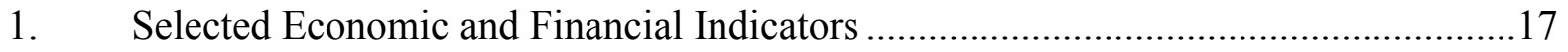

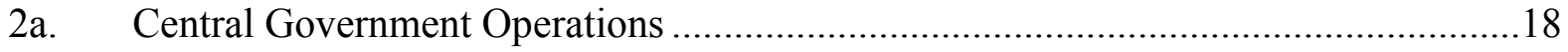

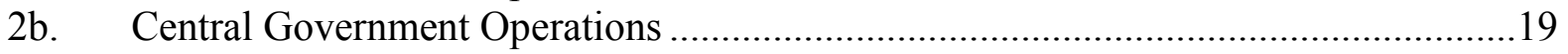

3. Summary Accounts of the Banking System ..........................................................20

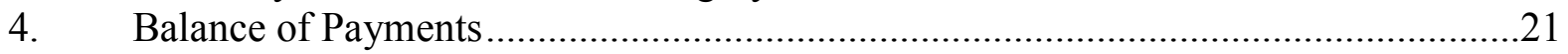

5. Financial Soundness Indicators of the Banking System ......................................22

6. Indicative Targets and Quantitative Performance Criteria, FY 2008 .......................23

7. Structural Performance Criteria and Benchmarks for the Fourth Program Review ....24

8. $\quad$ Proposed Schedule of Disbursements .................................................................25

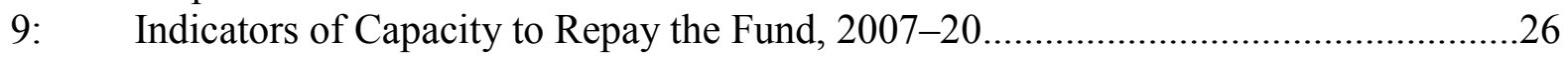

10. Status of HIPC Completion Point Triggers (January 2009) ….................................27

11. Indicators of External Vulnerability 2007-09 ..................................................28

\section{Figures}

1. Recent Economic Developments ...................................................................6

2. Bilateral and Effective Exchange Rates ............................................................ 7

\section{Boxes}

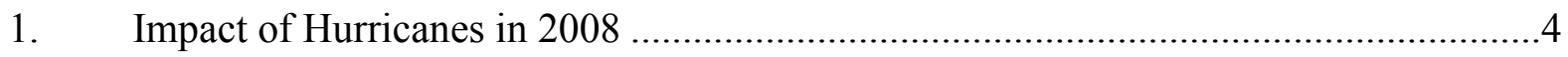

2. Emergency Law and Use of PetroCaribe Resources ............................................10

Attachments

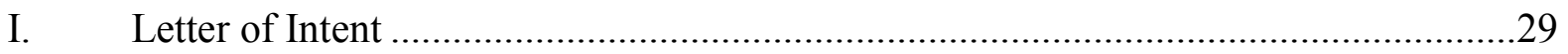

II. Memorandum on Economic and Financial Policies ...............................................32

III. Technical Memorandum of Understanding .....................................................43 


\section{Executive Summary}

Macroeconomic outcomes in the second year of the PRGF arrangement (FY 2008) were weaker than anticipated at the time of the third review. A prolonged political stalemate constrained government operations, while severe natural disasters caused damages estimated at about 15 percent of GDP. Economic growth slowed to 1.3 percent, and end-period inflation picked up to almost 20 percent. Despite these shocks, program performance through end-September 2008 remained satisfactory, avoiding a significant deterioration in macroeconomic stability.

The authorities are confronting difficult challenges in FY 2009. Responding to the humanitarian crisis and rebuilding infrastructure will require substantial resources, in addition to already large PRSP spending needs. Growth is expected to remain modest, at 2.5 percent, driven by public sector spending.

Haiti's external position is expected to deteriorate further in FY 2009, prompting the authorities to request a second program augmentation of 30 percent of quota. Given the exceptional magnitude of the shocks, limited reserve coverage, and the policy response of the authorities to maintain stability in extremely challenging circumstances, staff supports the request. The proposed augmentation, to be phased in two disbursements, would raise access to the PRGF ceiling of 140 percent of quota.

Priorities for the third and final program year are: (i) safeguarding macroeconomic stability in the face of large spending needs; (ii) finalizing outstanding structural reforms; and (iii) facilitating Haiti's timely progression to the HIPC completion point. Containing central bank financing to the government remains the main anchor of the program. In light of the large spending needs and limited pledged budget support, discussions on the macroeconomic framework for the last program year were more difficult than in previous years. In order to close the fiscal financing gap, the authorities had to scale back their spending plans significantly. Accumulated PetroCaribe resources were used to finance emergency reconstruction spending. Although off-budget, PetroCaribe spending is included in program parameters and will be strictly monitored by the authorities. The overall fiscal deficit is expected to widen from 2 percent of GDP in FY 2008 to 4 percent in FY 2009.

Program risks remain significant. Additional donor support to finance PRSP priorities and reconstruction would be crucial to alleviating spending pressures in an increasingly difficult political environment. Parliamentary elections in April 2009 could delay the approval of laws included in program conditionality and HIPC triggers. However, the authorities have demonstrated their commitment to maintaining macroeconomic stability and implementing their reform agenda, even in the face of great challenges. 


\section{INTRODUCTION}

1. Haiti recently experienced a series of devastating shocks that threatened macroeconomic stabililty and hindered growth. Riots over rising food and fuel prices prompted the resignation of the Prime Minister in April, leading to a five-month political stalemate that severely constrained government operations. ${ }^{1}$ Haiti was also hit by four backto-back hurricanes and tropical storms in August/September, which have caused extensive food shortages and damages to infrastructure estimated at about 15 percent of GDP (Box 1).

\section{Box 1. Impact of Hurricanes in 2008}

Four back-to-back storms in August/September caused damages and losses across the country estimated at close to US\$900 million (15 percent of GDP), the worst humanitarian disaster to hit Haiti over 100 years. Most affected were agriculture, housing, and transportation infrastructure. Post-disaster needs are about US\$763 million, including one-third (US\$269 million) needed for immediate relief, with the rest financing recovery of agricultural output and reconstruction of housing and physical infrastructure (Table).

The country's food security situation worsened substantially. A total of 3.3 million people are estimated to be food insecure, with pockets of acute food insecurity in some areas (about 210,000 people). The World Food Program is currently reaching 646,926 direct beneficiaries across the country, and donors are also increasing existing food-for-work and cash-forwork activities. The United Nations launched a flash appeal for humanitarian aid needs amounting to US\$127.5 million, for which donors have pledged/disbursed only about 45 percent to date.

\begin{tabular}{lc}
\hline \multicolumn{1}{c}{ Sector } & $\begin{array}{c}\text { Damages \& Losses } \\
\text { (US\$ mn) }\end{array}$ \\
\hline Total & 898 \\
Productive Sectors & 443 \\
Agriculture & 198 \\
Industry & 104 \\
Commerce & 119 \\
Tourism & 21 \\
Infrastructure & 151 \\
Roads & 130 \\
Water \& Sanitation & 18 \\
Social Sectors & 222 \\
Housing & 178 \\
Health \& Education & 44 \\
\hline Source: United Nations. & \\
&
\end{tabular}

\section{The impact of the international financial crisis represents another shock in the} making. Haiti has extremely shallow financial markets and, thus, has not experienced capital account effects. However, the slowdown in the United States and Canada is already affecting Haiti through lower export demand and fewer remittances. While Haiti's export sector is relatively small (less than 10 percent of GDP), private consumption is highly dependent on remittances (about 19 percent of GDP in 2008).

\section{The easing of international food and fuel prices only partly mitigates these new} shocks. Food prices and import needs are still high, with distribution networks impaired by the hurricanes and 60 percent of the fall harvest destroyed. Three new power plants are beginning operations, increasing volumes of fuel imports.

\footnotetext{
${ }^{1}$ The political crisis ended in early September, when Parliament ratified President Préval's third proposed candidate for Prime Minister, Ms. Michele Pierre-Louis, and her coalition government.
} 


\section{RECENT ECONOMIC DEVELOPMENTS}

\section{As a result of the shocks, macroeconomic outcomes in FY 2008 (October} 2007-September 2008) were weaker than anticipated at the time of the third review. Official estimates indicate that real GDP growth slowed to 1.3 percent from 3.4 percent in FY 2007, turning negative in per capita terms (after three consecutive years of positive growth). Twelve-month inflation peaked at 19.8 percent in September 2008, up from 7.9 percent a year earlier, but declined to 10.1 percent by end-December, owing to rapidly falling international food and fuel prices (Table 1 and Figure 1). After some small depreciation earlier in the year, the real effective exchange rate appreciated by about 5 percent during the last quarter of FY 2008, while the Gourde remained broadly stable against the U.S. dollar (Figure 2).

5. Budget execution in FY 2008 was satisfactory. Tax revenue was slightly below target (9.9 percent of GDP vs. 10.6 percent programmed), mostly owing to revenue losses (0.7 percent of GDP) from the temporary suspension of fuel price adjustments in response to rising commodity prices. ${ }^{2}$ Food and fuel price subsidies were however discontinued in August-October 2008. Spending capacity improved, with domestically-financed investment outlays exceeding expectations. The overall fiscal deficit (excluding grants and foreignfinanced projects) exceeded program projections somewhat ( 2 percent of GDP instead of 1.7 percent), and was fully financed with external resources (Tables $2 a, 2 b$ ).

6. Despite sterilization operations, base money increased more than targeted. Base money growth was 14 percent (y/y) by end-September 2008 (compared with 7.9 percent in the program), due to larger than programmed demand for currency (consequence of the higher than programmed inflation rate), as well as an increase in bank reserves that reflected, in part, the PetroCaribe-fueled accumulation of government deposits in commercial banks. In order to sterilize the increase in credit to the government during most of the second part of the fiscal year, and to smooth out exchange rate fluctuations, the BRH sold foreign exchange (about US\$52 million), stepped up the placements of bonds by more than G 1.5 billion (almost 20 percent) and doubled nominal interest rates. Credit to the private sector, which expanded in part due to the importers' increased credit demand to finance rising commodity prices, remained relatively low, at 13.1 percent of GDP (Table 3).

\footnotetext{
2 The authorities revised real and nominal GDP for FY 2007 and FY 2008, complicating comparisons between the FY 2008 program and preliminary outcomes. Using the program's nominal GDP, tax revenue in FY 2008 was 10.3 percent of GDP (Table $2 \mathrm{~b}$ ).
} 
Figure 1. Haiti: Recent Economic Developments

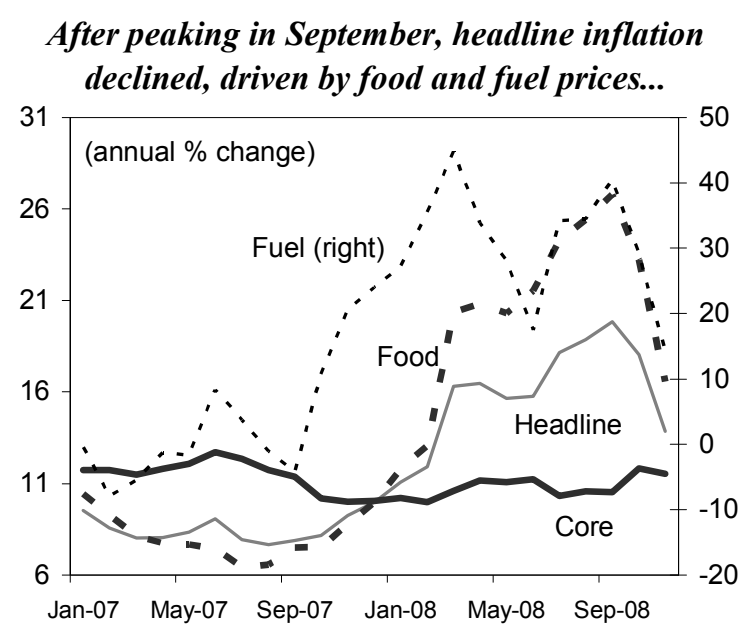

Expenditure execution, including capital spending, is on the rise.

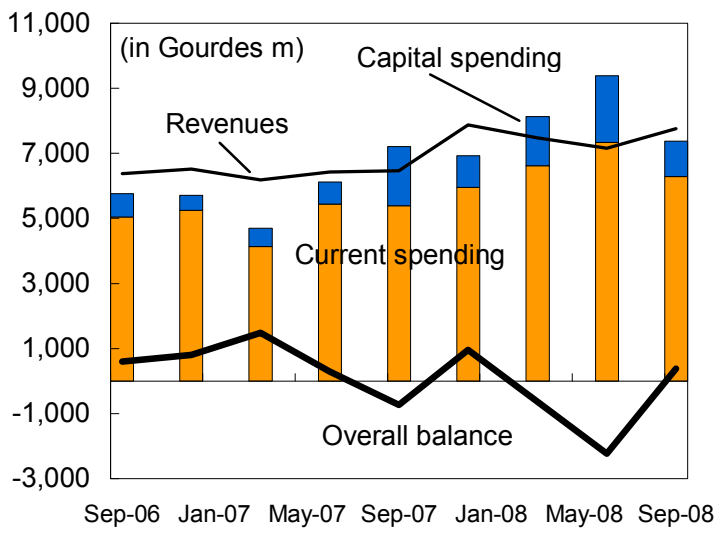

Although the current account balance has deteriorated due to the recent price shock...

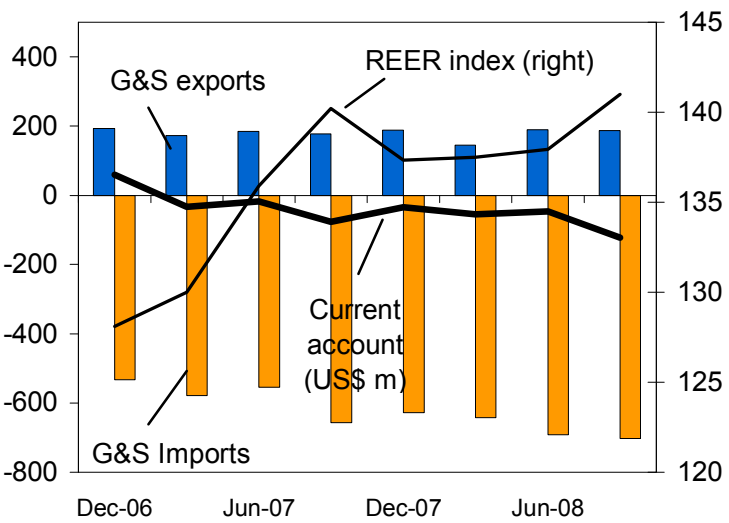

...as lower international prices are passedthrough to domestic prices.

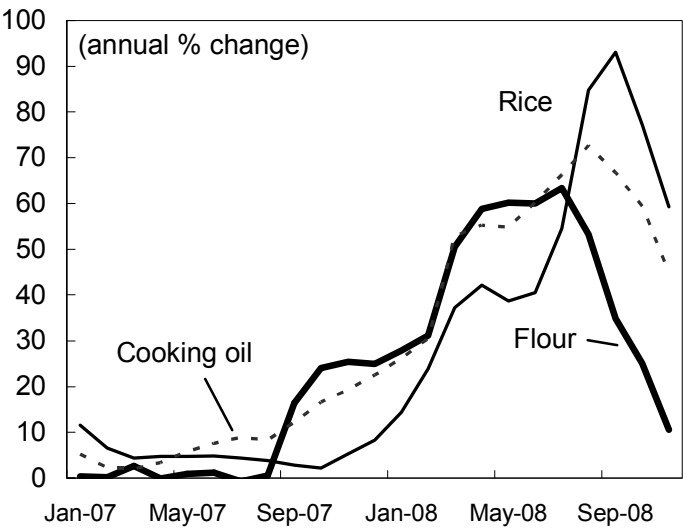

Nominal interest rates and currency growth have not kept up with inflation.

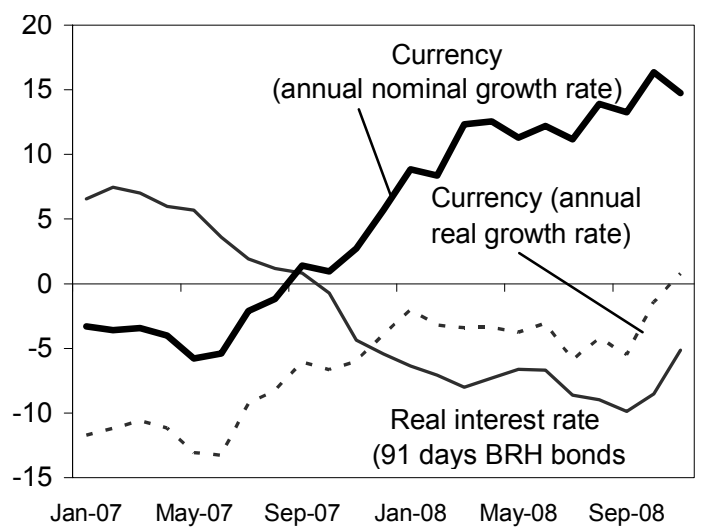

... NIR targets have been met comfortably, due in part to Petrocaribe-related flows

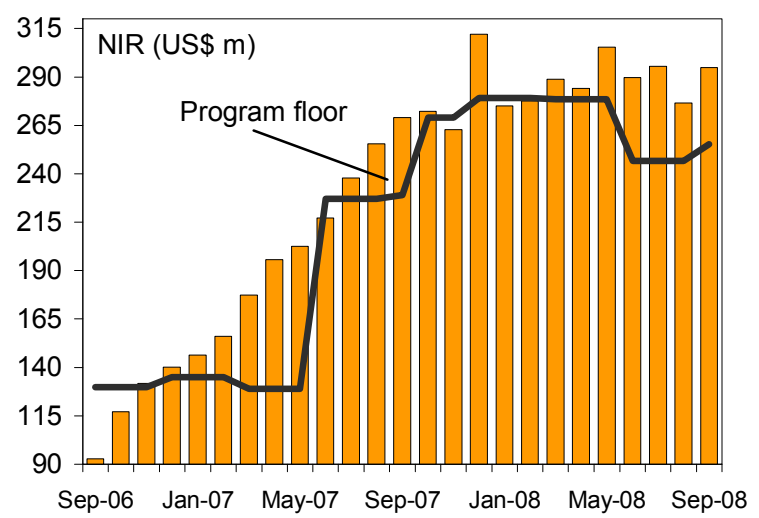

Sources: Haitian Authorities and IMF staff calculations. 


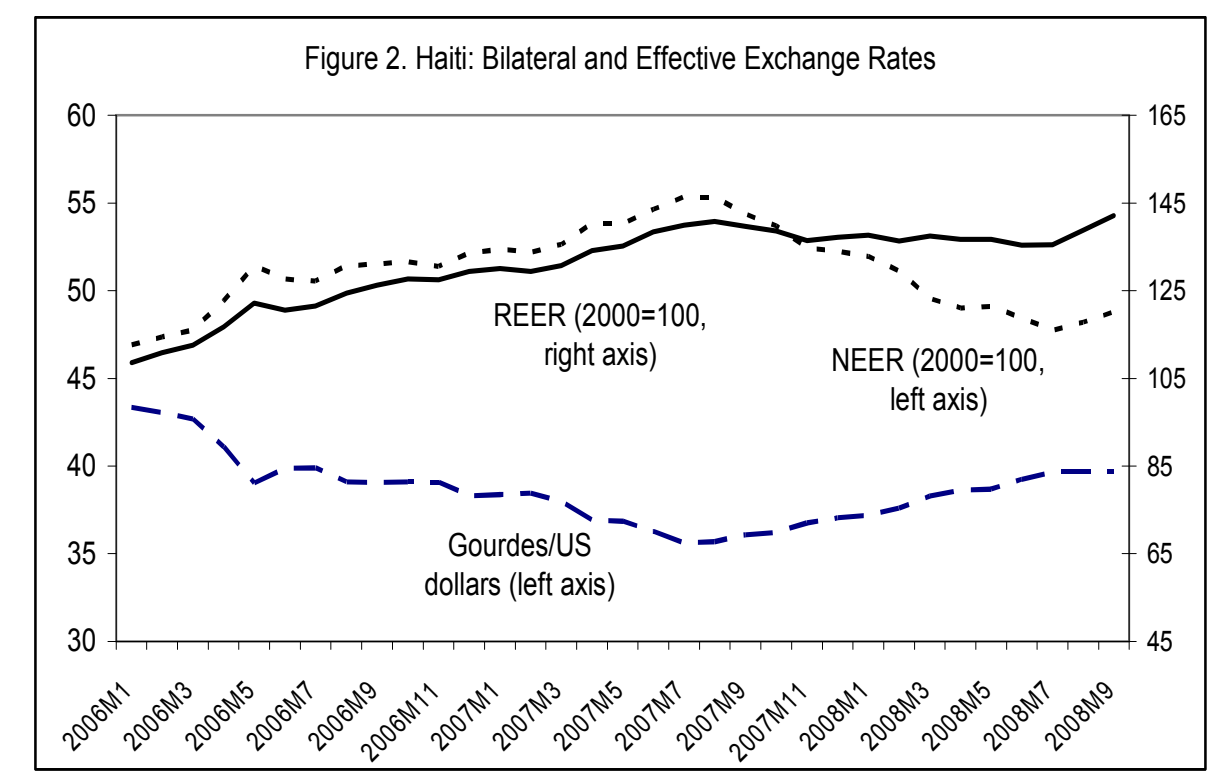

Source: IMF's Information Notice System.

7. Haiti's current account deficit widened to 2.6 percent of GDP. The trade deficit deteriorated by 7.5 percentage points of 2007 GDP ( $\$ 463$ million), largely because of higher food and fuel imports (up by $\$ 434$ million). The improved services balance was offset by lower current transfers than anticipated. The overall balance of payments remained in surplus (US\$41.5 million), due in part to PetroCaribe inflows used as budgetary support, but reserve coverage remained below 3 months of imports (Table 4).

\section{The financial system, which has not been significantly affected by the financial} crisis, remains sound. External credit lines are small and mostly trade-related. Indicators of banking sector soundness remained broadly satisfactory at end-September 2008, with increased net profits and declining non-performing loans, although the financial position of two small banks had weakened further (Table 5). An independent assessment indicated that the BNC (Banque Nationale de Crédit) will need to be recapitalized to accommodate the absorption of Socabank in 2006, and its operational structure reviewed (MEFP q23).

\section{Preliminary data for the first quarter of FY 2009 have been relatively positive.}

The exchange rate remained stable at about G 40 per U.S. dollar. Unadjusted NIR increased to about US\$350 mllion, largely reflecting transfers of PetroCaribe-related resources out of commercial banks and into the BRH. Gourde monetary base increased by about 15 percent at end-December (y-o-y), reflecting a fairly constant velocity (as cumulated annual inflation through December reached 10.1 percent while real GDP increased in FY 2008 by about 1.3 percent, as indicated above). Domestic tax collections for the first quarter were in line with expectations, while spending was boosted by higher reconstruction spending, payment 
of the traditional $13^{\text {th }}$ salary to civil servants, and higher transfers to the electricity company reflecting higher electricity production.

\section{Program Performance Through End-September 2008}

10. Performance against PRGF program targets at end-September was generally satisfactory, but maintaining macroeconomic stability in the face of severe shocks was particularly challenging:

- All but one quantitative performance criteria were met. The target for net BRH credit to the rest of the non-financial public sector was missed by G 229 million (about 0.5 percent of end-of-period BRH assets, or less than 0.1 percent of GDP). Staff supports a request for a waiver of this breached quantitative PC, as the deviation was minor and temporary. Preliminary information for Q1 FY 2009 indicates that this deviation is being reversed through a combination of increased deposits and some gross credit repayment. Performance criteria for net central bank credit to the central government, net domestic assets, and net international reserves were met, primarily due to the transfer of about US\$51 million in accrued PetroCaribe resources from commercial banks to the BRH toward the end of the fiscal year (MEFP 99 , Table 6).

- $\quad$ All structural PCs were met, but implementation of end-September benchmarks was mixed. The authorities prepared a strengthened plan to recapitalize the central bank and completed independent assessments of two systemically important commercial banks. Although progress was made on all program benchmarks, only one out of six benchmarks was fully completed (improving the regulatory framework and supervision of credit unions). Two benchmarks are being reset for end-March: (a) the publication of regular reports by the central bank (which has, however, stepped up monetary policy communications through speeches and interviews); and (b) a new organic law for the tax administration agency (DGI) which, although already drafted, has not yet been submitted to Parliament. The remaining benchmarks have been now completed: (i) three customs posts have become operational by end-December; (ii) a plan to improve systemic liquidity forecasting was finalized in January; and (iii) investment spending has been included in the public financial management

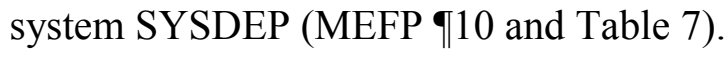

\section{Economic and Financial Policies for FY 2009}

\section{Discussions on the FY 2009 program were protracted, in light of significant} spending needs and limited resources, including budget support. Already large spending needs to intensify implementation of the PRSP have increased substantially following the natural disasters. With donor support committed so far insufficient to meet Haiti's needs, the authorities were forced to scale back their original spending plans significantly, and rely on accumulated PetroCaribe funds. A donors' conference, tentatively planned for early April 
2009, could help mobilize further resources, but prospects for significant additional commitments are uncertain. Key goals for the third program year will be to support public investment and poverty-reducing spending through increased domestic resources, while safeguarding macroeconomic stability and supporting progress to the HIPC completion point, tentatively scheduled for end-June 2009. Structural conditionality focuses on completing fiscal and financial sector reforms (MEFP $\$ 11)$.

\section{A. Macroeconomic Outlook}

12. The program framework includes revised goals for inflation and growth. Endperiod inflation has been set at 9.5 percent, and the growth projection has been revised downward from 4 percent to 2.5 percent. The recent drop in world commodity prices has begun to affect headline inflation, but the authorities consider that exchange rate depreciation, market rigidities and the damage to agriculture and to the distribution networks will likely prevent a faster decline (the original program goal was 7 percent). Growth should be boosted by increased public sector spending and investment. A faster pace would be impeded by the damage to infrastructure and agriculture, weaker private consumption reflecting lower remittances, and lower net exports related to the global downturn (MEFP q12-13).

\section{B. Fiscal Policy}

13. The fiscal program focuses on balancing large spending needs with principles of sound financing. The program overall fiscal deficit (excluding grants and foreign-financed projects) is projected to increase by 2 percentage points of GDP to 4 percent compared to FY 2008, with higher investment outlays financed by PetroCaribe resources, lower current spending and strengthened tax administration. For transparency, the program framework combines both on- and off-budget spending (Text Table 1 and MEFP \14).

14. Higher projected spending in FY 2009 addresses reconstruction needs as well as PRSP implementation, which should continue to guide fiscal policy in the medium term. However, the fiscal deficit (including grants) should start declining next year toward its more sustainable medium-term level of about 1.5 percent of GDP. Expenditure (excluding foreignfinanced projects) is programmed to increase by 2.7 percentage points of GDP to 14.6 percent. This includes 2.9 percent of GDP in off-budget emergency spending (Box 2). Current spending growth is limited. The 0.6 percentage point of GDP increase in the wage bill reflecting hirings of teachers and police, higher salaries for judges, and a one-off $14^{\text {th }}$ salary payment to civil servants, which was granted in lieu of a generalized salary increase for FY 2009, and transfers increase by 0.4 percentage point of GDP. Domestically-financed capital spending is projected to increase by 1.8 percentage points of GDP, to 3.9 percent (MEFP 115). The FY 2009 budget allocates substantial resources to social sectors and infrastructure. In line with PRSP priorities, total investment outlays focus on transport, lodging, employment, health, and food security. 


\section{Box 2. Haiti: Emergency Law and Use of PetroCaribe Resources}

Under an emergency law adopted following the hurricanes' devastation, the authorities decided to use US\$197.5 million in accumulated PetroCaribe resources to finance new off-budget emergency spending (US\$220.4 million including the one-off $14^{\text {th }}$ month salary payment to civil servants). While this spending is to be executed in FY 2009, the authorities already transferred in late September US\$51 million of PetroCaribe resources to the government accounts at the BRH to repay outstanding credit. The rest will be transferred in the course of FY 2009. Roughly 75 percent of the emergency spending will be on capital projects (2.2 percent of GDP), with the remainder for current expenditure ( 0.7 percent of GDP). Under the program, staff and the authorities agreed on specific measures to ensure transparency and oversight of the emergency spending.

\begin{tabular}{lr}
\hline Purpose & \% of total \\
\hline Restoring agricultural production (investment) & 16.7 \\
Spending for schools (50\% investment, 50\% transfers) & 12.4 \\
Health (investment) & 2.4 \\
Support to provinces (investment) & 8.1 \\
Purchase of equipment (investment) & 36.3 \\
Other investment (roads, sanitation, food, hydraulic and & 7.1 \\
electricity infrastructure, rehabilitation of prisons and police ) & 10.4 \\
14th month salary to civil servants (wages and salaries) & 0.8 \\
Other transfers & 5.9 \\
Administration (operations) &
\end{tabular}

Sources: Haitian authorities and IMF staff calculations.

\section{The budget proposes to finance higher expenditures through a combination of} strengthened customs and tax administration, and external support. Total revenue is projected to increase to 10.5 percent of GDP (from 9.9 percent of GDP in FY 2008). The introduction of the SYDONIA WORLD system to strengthen customs controls in the first half of FY 2009 (operational since December 1, 2008 in Port-au-Prince) and technical assistance from Canada and other development partners are projected to have a partial year effect in improving tax and customs administration (MEFP q17). Following widespread opposition by telecommunication companies, parliamentarians and the public, the authorities withdrew proposals for new telecommunications taxes and an increase in imports tariffs from the FY 2009 budget law, that would have increased revenue by an additional G 3 billion (almost 1 percent of GDP). ${ }^{3}$ The automatic fuel price adjustments to international prices were restored in October 2008. This was reflected in revenue projections for FY 2009, but lower world oil prices would result in a net decrease in the intake. Already committed budget

\footnotetext{
${ }^{3}$ The authorities intend to propose a revised package of tax measures as part of a supplementary budget in late Spring, following further studies and extensive consultations with stakeholders.
} 
support amounts to 3.1 percent of GDP (including PetroCaribe resources accumulated during FY 2008), and external project financing is projected at 5 percent of GDP.

16. The program includes US\$50 million in external budget support still to be identified, possibly in a donor's conference tentatively scheduled for early April. This will allow needed spending to get underway, while the authorities work to mobilize additional resources. If some or this entire amount does not materialize, a program adjustor of the same size would allow for limited and temporary new central bank financing to cover the shortfall. The authorities have committed to repay in FY 2010 any new central bank financing arising from shortfalls in budget support in FY 2009. As in the past, the program allows the authorities to spend any additional external resources received (MEFP $\$ 18$ ).

\section{The authorities are committed to strictly monitoring off-budget emergency}

spending. The off-budget spending will be reported to Parliament and subjected to the same auditing procedures by the national court of accounts. The authorities indicated that they were exploring options to channel new PetroCaribe/ALBA-related inflows during FY 2009 through a private binational Venezuela-Haiti corporation, but that discussions with Venezuela were still at a preliminary stage. In this light, and given uncertainties as to whether such flows will be continued, the program assumes no new PetroCaribe resources for the central government in FY 2009, but the TMU includes new adjusters to net international reserves, net BRH credit to the central government and net banking sector credit to the central government, should such new resources materialize (TMU qף27-28 and MEFP q20).

\section{Monetary Policy}

18. Monetary policy will focus on keeping core inflation reined in, as the impact of higher commodity prices wanes. To this end, the indicative FY 2009 target for base money growth was set at 9.3 percent, below projected nominal GDP growth. To help guide inflation expectations, the central bank will publish a quarterly report on monetary policy goals and outcomes beginning in early 2009. The BRH will continue to seek broader participation in the weekly central bank bond auctions and ensure close coordination with the Ministry of Economy and Finance to determine upcoming liquidity needs. The authorities may use reserves in order to smooth out exchange rate adjustment to equilibrate external imbalances caused by the shocks and to sterilize new temporary central bank financing if needed, but they are committed to maintaining a flexible exchange rate regime (MEFP |22).

19. Central bank independence is being strengthened. Implementation of the first stage of the central bank recapitalization plan, including higher interest payments by the government on the existing stock of central bank credit, should help increase the independence of monetary policy. Recently, legislation was passed to eliminate the central bank's legislatively-mandated involvement with SONAPI (industrial parks), APN (the port authority), and BPH, a small state-owned bank (MEFP 934 ). 


\section{External Sector and Debt Sustainability}

20. Haiti's overall balance of payments is expected to turn negative in FY 2009 for the first time since FY 2003. The projected deficit of about 2.2 percent of GDP reflects in part a worsening current account deficit (from 2.6 percent of GDP to 4.4 percent), as lower commodity prices are expected to be offset by a sharp increase in imports to address food shortages, infrastructure rehabilitation, and reconstruction needs. The economic downturn in the U.S. and Canada is expected to affect remittance and export receipts

\begin{tabular}{|c|c|c|c|c|}
\hline \multicolumn{5}{|c|}{$\begin{array}{l}\text { Text Table 1. Haiti: Natural Disaster and Global Slowdown } \\
\text { Impact on Key Balance of Payments Items } \\
\text { (In million U.S. dollars unless noted otherwise) }\end{array}$} \\
\hline & 2007 & $\begin{array}{r}2008 \\
\text { (a) }\end{array}$ & $\begin{array}{r}2009 \\
\text { (b) }\end{array}$ & $\begin{array}{l}2009 \text { vs. } 2008 \\
\text { (b) - (a) }\end{array}$ \\
\hline $\begin{array}{l}\text { Fuel Imports } \\
\% \text { of GDP }\end{array}$ & $\begin{array}{c}-415.0 \\
-6.8\end{array}$ & $\begin{array}{r}-602.2 \\
-8.5\end{array}$ & $\begin{array}{r}-426.4 \\
-5.6\end{array}$ & 175.8 \\
\hline $\begin{array}{l}\text { Food Imports } \\
\% \text { of GDP }\end{array}$ & $\begin{array}{r}-369.8 \\
-6.0\end{array}$ & $\begin{array}{r}-616.9 \\
-8.7\end{array}$ & $\begin{array}{r}-588.7 \\
-7.8\end{array}$ & 28.1 \\
\hline $\begin{array}{l}\text { Machinery and Transport Imports } \\
\% \text { of GDP }\end{array}$ & $\begin{array}{r}-234.8 \\
-3.8\end{array}$ & $\begin{array}{r}-187.7 \\
-2.6\end{array}$ & $\begin{array}{r}-393.3 \\
-5.2\end{array}$ & -205.6 \\
\hline $\begin{array}{l}\text { Net Services } \\
\% \text { of GDP }\end{array}$ & $\begin{array}{r}-443.6 \\
-7.2\end{array}$ & $\begin{array}{r}-385.3 \\
-5.4\end{array}$ & $\begin{array}{r}-474.1 \\
-6.2\end{array}$ & -88.8 \\
\hline $\begin{array}{l}\text { Assembly Exports } \\
\% \text { of GDP }\end{array}$ & $\begin{array}{r}180.6 \\
2.9\end{array}$ & $\begin{array}{r}165.1 \\
2.3\end{array}$ & $\begin{array}{r}168.5 \\
2.2\end{array}$ & 3.4 \\
\hline $\begin{array}{r}\text { Remittances } \\
\% \text { of GDP }\end{array}$ & $\begin{array}{c}1,125.7 \\
18.3\end{array}$ & $\begin{array}{c}1,369.7 \\
19.3\end{array}$ & $\begin{array}{c}1,215.3 \\
16.0\end{array}$ & -154.4 \\
\hline $\begin{array}{l}\text { Total Current Account Impact } \\
\% \text { of } 2009 \text { GDP }\end{array}$ & & & & $\begin{array}{l}-241.4 \\
-3.2\end{array}$ \\
\hline
\end{tabular}

Sources: Haitian authorities; and Fund staff estimates.

(Text Table 1 provides a detailed breakdown of key current account items from 2007 through 2009 while Table 4 shows how the balance of payments has deteriorated relative to projections at the time of the third review). The capital and financial account is also assumed to weaken, primarily due to lower projected public sector loan disbursements. ${ }^{4}$ Private investment is expected to be very low, because of the global environment and the more uncertain domestic and external situation of the country. Under the program, the authorities would be allowed to use up to US\$50 million in NIR to sterilize central bank financing in the event of delays in external budget support.

\section{To help cover the projected balance of payments gap, the authorities are} requesting a second program augmentation equivalent to 30 percent of quota (SDR 25 million). Reserve coverage, which reached 2.9 months of imports in 2008, would fall to 2.6 months in the absence of the additional assistance requested from the Fund, and to 2.5 months without the Fund augmentation and the additional budget support highlighted in Text table 2. The Fund augmentation and this additional budget support would bring reserve coverage to 2.8 months of imports in 2009. The proposed augmentation would be provided in two tranches: 20 percent of quota upon completion of the current review, and the rest upon completion of the fifth review (Table 8). These purchases would bring Haiti to the normal maximum PRGF access level of 140 percent of quota.

\footnotetext{
${ }^{4}$ The sharp drop in public sector loan disbursements shown in Table 4 is partly explained by the absence of PetroCaribe flows to the government in 2009. There has also been a shift in external support from loans to grans, but the increase in official transfers in 2009 only partly offsets lower levels of official lending.
} 
22. The proposed augmentation would complement the budget support, project financing, and humanitarian assistance already committed by other stakeholders, although pledges remain well below estimated needs (Text Table 2). In response to the hurricanes, the IDB and the European Union intend to increase their budget support for 2009. ${ }^{5}$ The World Bank has committed additional grants of US\$25 million (of which US\$5 million in budget support), and

Text table 2. Haiti-Burdensharing by Donors (In million U.S. dollars)

\begin{tabular}{lcc}
\hline & $\begin{array}{c}\text { Additional Support } \\
\text { Pledged }\end{array}$ & $\begin{array}{c}\text { Total } \\
\text { FY2009 support }\end{array}$ \\
\hline Total Pledges & 193.5 & 654.4 \\
$\begin{array}{l}\text { Budget support 1/ } \\
\begin{array}{l}\text { Project financing and } \\
\text { humanitarian aid }\end{array}\end{array}$ & 36.4 & 92.4 \\
\hline
\end{tabular}

1/ Petrocaribe budget support is booked in the BOP in FY2008.

bilateral donors have committed substantial aid, mainly for humanitarian relief.

23. Haiti's capacity to repay the Fund will remain adequate despite the proposed increase in access. Debt service to the Fund will equal 0.23 percent and 0.20 percent of domestic revenues and exports of goods and services, respectively, on average over the next four years, and should remain manageable through 2020 (Table 9).

24. An update of the LIC debt sustainability analysis suggests that the PetroCaribe resources received in FY 2008 and the proposed augmentation of the PRGF arrangement will adversely affect the debt trajectory, but not alter the main conclusions of the last LIC DSA. The NPV of debt-to-exports ratio remains over the threshold in the baseline and shock scenarios, but all other indicators remain below their respective thresholds. This reflects Haiti's relatively small export sector: the current account is generally financed through transfers. The NPV of debt-to-exports ratio remains below the relevant threshold when HIPC/MDRI relief is assumed, but with little cushion in the likely event of future shocks.

\section{E. Structural Policies}

25. The structural agenda in the final program year will focus on finalizing pending reforms initiated earlier in the program. The proposed four structural PCs and five benchmarks are critical for achieving the program objectives of strengthening fiscal governance and soundness, fostering banking sector stability, and increasing the independence of monetary policy (MEFP Table 3).

26. The authorities are working on completing a few remaining HIPC triggers to reach the completion point by mid-2009 (Table 10). The main challenge will be to secure

\footnotetext{
${ }^{5}$ The IDB will provide additional interim debt relief of close to US $\$ 15$ million in 2009 . The debt relief estimate in the balance of payments remains roughly unchanged from the last review, because the World Bank cannot provide further interim relief in FY 2009. Haiti already benefited from an increase of the limitation of World Bank interim relief from one third to 50 percent of the maximum NPV amount following satisfactory progress in completion point triggers' implementation.
} 
prompt approval of the new procurement law, which will be submitted to Parliament in January 2009, as a six-month implementation period of the approved law is needed to meet the trigger. Staff anticipates that all other triggers will be met.

\section{F. Program Monitoring}

27. The program monitoring framework has been altered to take into account the use of PetroCaribe resources. Part of the PetroCaribe resources received in FY 2008 (about US\$200 million) were transferred in FY 2008 (US\$51 million) and the remainder will be transferred in FY 2009 (US\$149 million). In both years, the transferred resources are treated as external budget support for program purposes. The TMU has been revised to ensure that any such future use for central government spending (on- or off-budget) is treated as budget support, and thus counts as part of the adjustment for net program external financing (TMU I29-30). Furthermore, the performance criteria on NIR and net domestic banking sector credit to the government are adjusted to take into account the drawdown in PetroCaribe deposits at the Central Bank (TMU $\mid 27-28-31)$.

28. The FY 2009 program will use the same quantitative performance criteria as in the first two program years (MEFP, Table 2). The program will be monitored on a quarterly basis, with test dates at end-March and end-September 2009 for NIR, NDA, central bank financing to the public sector, concessionality of external debt, and domestic and external arrears accumulation. The program provides some room for BRH credit to the government in the first two quarters, which are likely to be the most critical from a humanitarian and reconstruction perspective. The BRH credit is programmed to be reversed by end-September. Similarly, use of NIR for the fiscal year will be frontloaded to allow flexibility for the BRH in addressing volatility in the foreign exchange market and sterilizeif needed-temporary BRH financing.

\section{Program Risks}

29. Despite the authorities' strong track record and commitment to prudent macroeconomic policies, program risks are substantial and have increased since the last review. Safeguarding the significant macroeconomic gains of the past four years will not be easy amid high external vulnerability (Table 11). The main risks are:

- $\quad$ Political and social instability and weather-related shocks, that are ever-looming. In particular, parliamentary elections in April 2009 and an ongoing constitutional debate during the year may cause further delays in approval of key reform legislation;

- $\quad$ the uncommitted budget support may not be forthcoming;

- $\quad$ the downturn in the United States and Canada could lead to a sharper decline in exports and transfers; and 
- $\quad$ spending pressures may emerge if programmed spending is insufficient to cover needs, and/or in the run-up to parliamentary elections in April 2009. The early withdrawal of the proposed new taxes sets an unfavorable precedent in terms of the authorities' capacity to introduce further revenue measures.

\section{StAfF APPRAisAL}

30. Performance in the second program year was satisfactory, but maintaining macroeconomic stability was difficult amid the severe shocks. Economic growth has been weaker than expected, challenging the authorities' ability to implement prudent fiscal and monetary policies. Nevertheless, spending and core inflation were kept broadly under control. Implementation of structural measures slowed as a result of the shocks, although progress was made in all areas.

31. The recent devastating natural disasters compounded by the global slowdown present numerous challenges for macroeconomic policies. The program is pragmatic in terms of safeguarding economic stability while maintaining a focus on continued economic and social progress in Haiti. The authorities are encouraged to make the most of the donor support for strengthening tax administration, and should work closely with donors to mobilize additional external assistance. Higher projected spending in FY 2009 addresses reconstruction needs as well as intensified PRSP implementation. Staff urges the authorities to ensure full transparency and close monitoring of off-budget emergency spending, and to resist pressures for further spending unless additional domestic revenue or external financing becomes available.

\section{Monetary policy should focus on containing core inflation and preventing} excessive exchange rate volatility. Headline inflation could decline fairly rapidly if WEO commodity price forecasts materialize, but the authorities will need to monitor closely developments in core inflation. They should also be prepared to sterilize temporary central bank financing to the government as needed. The banking sector appears generally sound, but the impact of recent natural disasters and slowdown of the economy pose risks to the quality of credit portfolios that need to be closely monitored.

33. Further efforts will be needed to safeguard debt sustainability. Given the limited margin below the threshold even after anticipated debt relief, maintaining debt sustainability over the medium-term will require that the authorities take a prudent approach to borrowing, even on concessional terms, and adopt policies to promote growth and export diversification.

34. Program risks have risen, but continued Fund involvement is justified by the authorities' commitment and will help safeguard the hard-won and significant gains of the past four years. The proposed augmentation and additional commitments from other institutions can help the country get through the exceptionally difficult current circumstances, 
and reach its HIPC completion point. Staff urges the authorities to ensure a timely implementation of remaining completion point triggers.

35. Staff supports the requested conclusion of the fourth review, waiver, and augmentation of access under the arrangement. Performance has been satisfactory, and the deviation under the performance criterion has been minor. Given pressure on the balance of payments caused by the devastating natural disasters and the global slowdown, low reserve coverage, the government's past strong performance and commitment to the program, the manageable level of outstanding Fund credit, and Haiti's track record of repayment, the staff believes that an augmentation of access under the PRGF arrangement by 30 percent of quota is appropriate. 
Table 1. Haiti: Selected Economic and Financial Indicators

(Fiscal year ending September 30)

Nominal GDP (2008): US\$ 7.11 billion

Population (2008): 9.76 million

Share of pop. living with less than $\$ 1$ a day (2003): 54 percent
GDP per capita (2008): US\$728

Adult literacy (2008): 53 percent

Unemployment rate (2003): 27 percent

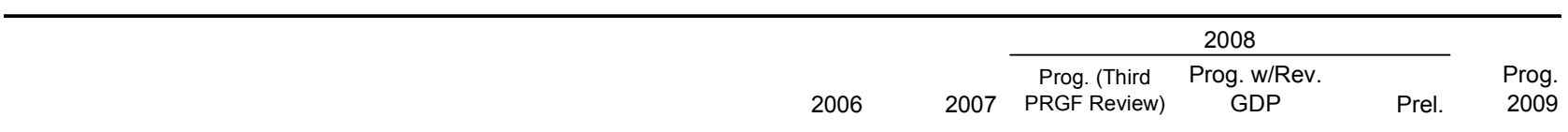

(change over previous year unless otherwise stated)

\section{National income and prices \\ GDP at constant prices \\ GDP deflator \\ Consumer prices (period average) \\ Consumer prices (end-of-period)}

\section{External sector}

Exports (f.o.b.)

Imports (f.o.b.)

Real effective exchange rate (+ appreciation)

Central government

Total revenue and grants

Total revenue excl. grants

Current expenditure

Total expenditure

\section{Money and credit}

Credit to the nonfinancial public sector (net) $1 /$

Credit to private sector

Base money

Broad money (incl. foreign currency deposits)

\section{Central government}

Overall balance

Overall balance (excl. grants)

Overall balance (excl. grants and externally-financed projects)

Overall balance (excl. ext.-financed projects and project grants)

Central bank net credit to the central government

\section{Savings and investment}

Gross investment

Gross national savings

Of which: Central government savings

External current account balance (incl. official grants)

External current account balance (excl. official grants)

External public debt (end-of-period)

Total public debt (end-of-period) 2/

External public debt service (in percent of

exports of goods and nonfactor services) $3 /$

Overall balance of payments

Net international reserves (program) 4/

Liquid gross reserves $5 /$

In months of imports of the following year

Exchange rate (gourdes per dollar, end-of-period)

Nominal GDP (millions of gourdes)

Nominal GDP (millions of U.S. dollars)

\begin{tabular}{|c|c|c|c|c|c|}
\hline 2.3 & 3.4 & 2.5 & 2.5 & 1.3 & 2.5 \\
\hline 16.6 & 10.7 & 14.5 & 14.5 & 17.0 & 12.0 \\
\hline 14.2 & 9.0 & 14.5 & 14.5 & 14.4 & 12.8 \\
\hline 12.4 & 7.9 & 16.0 & 16.0 & 19.8 & 9.5 \\
\hline 7.7 & 5.7 & -6.0 & -6.0 & -6.2 & \\
\hline 18.3 & 4.5 & 24.3 & 24.3 & 30.2 & \\
\hline 10.5 & 14.9 & & $\ldots$ & $\ldots$ & \\
\hline 20.2 & 30.7 & 29.9 & 29.9 & 9.0 & 29 \\
\hline 23.7 & 15.4 & 21.3 & 21.3 & 15.7 & 22 \\
\hline-0.7 & -2.0 & 54.5 & 54.5 & 41.5 & 24 \\
\hline 13.4 & 14.6 & 44.4 & 44.4 & 32.7 & 34 \\
\hline-4.9 & -6.9 & 0.0 & 0.0 & -29.8 & 48 \\
\hline 5.5 & 10.8 & 12.3 & 12.3 & 25.2 & 15 \\
\hline 5.5 & 7.6 & 7.9 & 7.9 & 13.9 & 9 \\
\hline 10 & 4.8 & 10.6 & 10.6 & 17.7 & 11 \\
\hline
\end{tabular}

(in percent of GDP, unless otherwise stated)

$\begin{array}{rrrrrr}-1.7 & 0.2 & -1.6 & -1.6 & -2.8 & -3.9 \\ -4.9 & -4.8 & -7.8 & -7.6 & -6.8 & -9.1 \\ -0.5 & 0.3 & -1.7 & -1.7 & -2.0 & -4.0 \\ -0.2 & 1.5 & 0.0 & 0.0 & -0.7 & -2.8 \\ -0.2 & -0.4 & 0.0 & 0.0 & 0.0 & 0.1 \\ & & & & & \\ 28.9 & 27.7 & 27.7 & 26.9 & 26.0 & 31.0 \\ 27.4 & 27.4 & 24.7 & 24.0 & 23.4 & 26.5 \\ 0.8 & 3.1 & 1.7 & 1.6 & 1.3 & 1.1 \\ -1.4 & -0.3 & -3.0 & -2.9 & -2.6 & -4.4 \\ -9.3 & -6.6 & -10.0 & -9.7 & -8.6 & -11.9 \\ 29.7 & 29.1 & 23.6 & 22.9 & 26.7 & 25.5 \\ 33.5 & 32.9 & 27.0 & 26.3 & 30.2 & 27.3 \\ & & & & & \end{array}$

(in millions of US\$, unless otherwise stated)

\begin{tabular}{rrrrrr}
79.1 & 163.4 & -49.9 & -49.9 & 41.5 & -158.3 \\
92.7 & 269.1 & 244.7 & 244.7 & 287.4 & 237.4 \\
337.1 & 544.7 & 578.1 & 578.1 & 707.8 & 696.0 \\
1.7 & 2.3 & 2.4 & 2.4 & 2.9 & 2.8 \\
39.1 & 36.4 & $\ldots$ & $\ldots$ & $\ldots$ & $\ldots$ \\
200,456 & 229,538 & 264,722 & 271,971 & 271,971 & 312,222 \\
4,836 & 6,137 & 6,966 & 7,108 & 7,108 & 7,589 \\
\hline
\end{tabular}

Sources: Ministry of Economy and Finance; Bank of the Republic of Haiti; Fund staff estimates; and World Bank.

1/ In FY2008 it reflects accumulation of Petrocaribe-related resources; in FY2009, it reflects the use of Petrocaribe-related resources accumulated in FY2008.

2/ Includes external public sector debt, outstanding central bank bonds, and credit from commercial banks to the NFPS.

It does not reflect possible completion point debt reduction in 2009

3 / Based on originally scheduled debt service, not incl debt relief.

4/ Excluding commercial bank forex deposits, letters of credit, guarantees, and earmarked project accounts.

5/ Gross Liquid International Reserves for FY2009 assume the disbursement of the proposed US \$ 37 million augmentation.

6/ GDP ratios are calculated using Nominal Program Figures for FY08 (numerator) and actual nominal GDP. 
Table 2a. Haiti: Central Government Operations

(Fiscal year ending September 30; in millions of gourdes)

\begin{tabular}{|c|c|c|c|c|c|}
\hline & \multirow[b]{2}{*}{2006} & \multirow[b]{2}{*}{2007} & \multicolumn{2}{|l|}{2008} & \multirow{2}{*}{$\begin{array}{l}\text { Prog. } \\
2009\end{array}$} \\
\hline & & & Prog. (Third PRGF Review) & Prel. & \\
\hline Total revenue and grants & 26,558 & 34,713 & 44,386 & 37,843 & 48,840 \\
\hline Domestic revenue & 20,110 & 23,197 & 28,146 & 26,849 & 32,781 \\
\hline Domestic taxes & 12,878 & 15,740 & 18,788 & 18,026 & 22,035 \\
\hline Customs duties & 6,099 & 6,828 & 8,622 & 7,917 & 10,463 \\
\hline Other current revenue & 1,133 & 629 & 736 & 906 & 284 \\
\hline Grants & 6,449 & 11,517 & 16,240 & 10,994 & 16,060 \\
\hline Budget support & 684 & 2,720 & 4,600 & 3,426 & 3,814 \\
\hline Project grants & 5,765 & 8,797 & 11,640 & 7,568 & 12,245 \\
\hline Total expenditure $1 /$ & 29,890 & 34,248 & 48,695 & 45,442 & 61,141 \\
\hline Current expenditure & 19,242 & 18,864 & 28,325 & 26,697 & 33,173 \\
\hline Wages and salaries & 6,470 & 8,087 & 12,566 & 11,716 & 15,438 \\
\hline Net Operations 1/ & 6,167 & 3,027 & 8,022 & 8,178 & 8,362 \\
\hline Operations 21 & 4,505 & 6,322 & 6,754 & 7,350 & 8,362 \\
\hline Interest payments & 1,052 & 2,420 & 1,141 & 1,768 & 2,235 \\
\hline External & 209 & 720 & 869 & 928 & 1,046 \\
\hline Domestic & 843 & 1,700 & 272 & 840 & 1,189 \\
\hline Transfers and subsidies & 5,553 & 5,330 & 6,596 & 5,035 & 7,138 \\
\hline of which Rice subsidy & & & & 681 & 0 \\
\hline Capital expenditure & 10,648 & 15,385 & 20,370 & 18,745 & 27,967 \\
\hline Domestically financed & 1,940 & 3,546 & 4,314 & 5,611 & 12,225 \\
\hline Foreign-financed & 8,708 & 11,839 & 16,056 & 13,134 & 15,742 \\
\hline Overall balance & $-3,332$ & 465 & $-4,309$ & $-7,599$ & $-12,300$ \\
\hline Excl. grants & $-9,781$ & $-11,052$ & $-20,549$ & $-18,593$ & $-28,360$ \\
\hline Excl. grants and externally financed projects & $-1,073$ & 787 & $-4,492$ & $-5,459$ & $-12,617$ \\
\hline Excl. project grants and ext. financed projects & -389 & 3,507 & 107 & $-2,033$ & $-8,803$ \\
\hline Financing & 3,332 & -465 & 4,309 & 7,599 & 12,300 \\
\hline External net financing & 4,038 & -106 & 3,743 & 6,607 & 9,793 \\
\hline Loans (net) & 3,719 & 1,620 & 3,363 & 6,607 & 7,736 \\
\hline Disbursements & 3,719 & 3,406 & 5,156 & 8,283 & 9,547 \\
\hline Budget support & 776 & 364 & 739 & 2,716 & 6,050 \\
\hline of which Petrocaribe & $\ldots$ & & $\ldots$ & 1,772 & 6,030 \\
\hline Project loans & 2,943 & 3,042 & 4,416 & 5,566 & 3,497 \\
\hline Amortization & 0 & $-1,786$ & $-1,793$ & $-1,676$ & $-1,811$ \\
\hline External financing to be committed & $\ldots$ & & $\ldots$ & $\ldots$ & 2,057 \\
\hline Arrears (net) & 319 & $-1,726$ & 0 & 0 & 0 \\
\hline Internal net financing & -706 & $-1,264$ & -335 & 83 & 1,559 \\
\hline Banking system & -634 & $-1,264$ & 0 & -229 & 349 \\
\hline $\mathrm{BRH}$ & -344 & -949 & 0 & 121 & 349 \\
\hline Commercial banks & -290 & -315 & 0 & -349 & 0 \\
\hline Nonbank financing & -120 & 0 & -335 & 312 & 1,210 \\
\hline Arrears (net) & 48 & 0 & 0 & 0 & 0 \\
\hline Debt rescheduling & 0 & 134 & 158 & 163 & 161 \\
\hline HIPC interim relief & 0 & 771 & 743 & 747 & 787 \\
\hline Unidentified financing (in U.S. dollars) & 0 & 0 & 10 & 0 & 0 \\
\hline
\end{tabular}

Sources: Ministry of Finance and Economy; and Fund staff estimates

$1 /$ Commitment basis except for domestically financed capital expenditure, which is reported on cash basis from 2007 on. $2 /$ Includes stastical discrepancy. 
Table 2b. Haiti: Central Government Operations

(Fiscal year ending September 30; in percent of GDP)

\begin{tabular}{|c|c|c|c|c|c|c|}
\hline & & & & 2008 & & \\
\hline & 2006 & 2007 & $\begin{array}{c}\text { Prog. (Third } \\
\text { PRGF } \\
\text { Review) }\end{array}$ & $\begin{array}{l}\text { Prog. } \\
\text { w/Rev. GDP }\end{array}$ & Prel. & $\begin{array}{c}\text { Prog } \\
2009\end{array}$ \\
\hline Total revenue and grants & 13.2 & 15.1 & 16.8 & 16.3 & 13.9 & 15.6 \\
\hline Total revenue & 10.0 & 10.1 & 10.6 & 10.3 & 9.9 & 10.5 \\
\hline Domestic taxes & 6.4 & 6.9 & 7.1 & 6.9 & 6.6 & 7.1 \\
\hline Customs duties & 3.0 & 3.0 & 3.3 & 3.2 & 2.9 & 3.4 \\
\hline Other current revenue & 0.6 & 0.3 & 0.3 & 0.3 & 0.3 & 0.1 \\
\hline Grants & 3.2 & 5.0 & 6.1 & 6.0 & 4.0 & 5.1 \\
\hline Budget support & 0.3 & 1.2 & 1.7 & 1.7 & 1.3 & 1.2 \\
\hline Project grants & 2.9 & 3.8 & 4.4 & 4.3 & 2.8 & 3.9 \\
\hline Total expenditure $2 /$ & 14.9 & 14.9 & 18.4 & 17.9 & 16.7 & 19.6 \\
\hline Current expenditure & 9.6 & 8.2 & 10.7 & 10.4 & 9.8 & 10.6 \\
\hline Wages and salaries & 3.2 & 3.5 & 4.7 & 4.6 & 4.3 & 4.9 \\
\hline Net Operations 2/ & 3.1 & 1.3 & 3.0 & 2.9 & 3.0 & 2.7 \\
\hline Operations $3 /$ & 2.2 & 2.8 & 2.6 & 2.5 & 2.7 & 2.7 \\
\hline Interest payments & 0.5 & 1.1 & 0.4 & 0.4 & 0.7 & 0.7 \\
\hline Transfers and subsidies & 2.8 & 2.3 & 2.5 & 2.4 & 1.9 & 2.3 \\
\hline Of which: Rice subsidy & $\ldots$ & & 0.4 & 0.4 & 0.3 & \\
\hline Capital expenditure & 5.3 & 6.7 & 7.7 & 7.5 & 6.9 & 9.0 \\
\hline Domestically financed & 1.0 & 1.5 & 1.6 & 1.6 & 2.1 & 3.9 \\
\hline Foreign-financed & 4.3 & 5.2 & 6.1 & 5.9 & 4.8 & 5.0 \\
\hline Overall balance & -1.7 & 0.2 & -1.6 & -1.6 & -2.8 & -3.9 \\
\hline Excl. grants & -4.9 & -4.8 & -7.8 & -7.6 & -6.8 & -9.1 \\
\hline Excl. grants and externally financed projects & -0.5 & 0.3 & -1.7 & -1.7 & -2.0 & -4.0 \\
\hline Excl. project grants and ext. financed projects & -0.2 & 1.5 & 0.0 & 0.0 & -0.7 & -2.8 \\
\hline Financing & 1.7 & -0.2 & 1.6 & 1.6 & 2.8 & 3.9 \\
\hline External net financing & 2.0 & 0.0 & 1.4 & 1.4 & 2.4 & 3.1 \\
\hline Loans (net) & 1.9 & 0.7 & 1.3 & 1.2 & 2.4 & 2.5 \\
\hline Disbursements & 1.9 & 1.5 & 1.9 & 1.9 & 3.0 & 3.1 \\
\hline Budget support & 0.4 & 0.2 & 0.3 & 0.3 & 1.0 & 1.9 \\
\hline Of which: Petrocaribe & $\ldots$ & & & 0.0 & 0.7 & 1.9 \\
\hline Project loans & 1.5 & 1.3 & 1.7 & 1.6 & 2.0 & 1.1 \\
\hline Amortization & 0.0 & -0.8 & -0.7 & -0.7 & -0.6 & -0.6 \\
\hline External financing to be committed & $\ldots$ & $\ldots$ & 0.1 & 0.1 & 0.0 & 0.7 \\
\hline Arrears (net) & 0.2 & -0.8 & 0.0 & 0.0 & 0.0 & 0.0 \\
\hline Internal net financing & -0.4 & -0.6 & -0.1 & -0.1 & 0.0 & 0.5 \\
\hline Banking system & -0.3 & -0.6 & 0.0 & 0.0 & -0.1 & 0.1 \\
\hline $\mathrm{BRH}$ & -0.2 & -0.4 & 0.0 & 0.0 & 0.0 & 0.1 \\
\hline Commercial banks & -0.1 & -0.1 & 0.0 & 0.0 & -0.1 & 0.0 \\
\hline Other nonbank financing & -0.1 & 0.0 & -0.1 & -0.1 & 0.1 & 0.4 \\
\hline Arrears (net) & 0.0 & 0.0 & 0.0 & 0.0 & 0.0 & 0.0 \\
\hline Rescheduling & 0.0 & 0.1 & 0.1 & 0.1 & 0.1 & 0.1 \\
\hline HIPC interim relief & 0.0 & 0.3 & 0.3 & 0.3 & 0.3 & 0.3 \\
\hline Unidentified financing & 0.0 & 0.0 & 0.0 & 0.0 & 0.0 & 0.0 \\
\hline
\end{tabular}

Sources: Ministry of Finance and Economy; and Fund staff estimates

1/ GDP ratios are calculated using nominal program figures for FY08 (numerator) and actual nominal GDP (denominator). 2/ Commitment basis except for domestically financed capital expenditure, which is reported on cash basis from 2007 on. $3 /$ Includes stastical discrepancy. 
Table 3. Haiti: Summary Accounts of the Banking System (Fiscal year ending September 30; in millions of gourdes)

\begin{tabular}{|c|c|c|c|c|c|}
\hline & & & 2008 & & Prog. \\
\hline & 2006 & 2007 & Prog. (Third PRGF Review) & Prel. & 2009 \\
\hline & entral Ban & & & & \\
\hline Net foreign assets & 10,893 & 16,849 & 18,000 & 21,035 & 20,255 \\
\hline (In millions of U.S. dollars) & 279 & 463 & 474 & 526 & 478 \\
\hline Net international reserves (program) $1 /$ & 93 & 269 & 245 & 287 & 237 \\
\hline Commercial bank forex deposits & 158 & 181 & 206 & 222 & 222 \\
\hline Net domestic assets & 12,279 & 8,081 & 8,909 & 7,356 & 10,772 \\
\hline Credit to the nonfinancial public sector & 21,002 & 19,905 & 20,041 & 20,541 & 20,890 \\
\hline Of which: Credit to the central government & 21,436 & 20,487 & 20,487 & 20,607 & 20,956 \\
\hline Liabilities to commercial banks (excl gourde deposits) & $-13,986$ & $-15,596$ & $-17,204$ & $-18,459$ & $-15,067$ \\
\hline BRH bonds & $-7,809$ & $-9,013$ & $-9,384$ & $-9,601$ & $-5,677$ \\
\hline Counterpart of commercial bank forex deposits & $-6,177$ & $-6,583$ & $-7,820$ & $-8,858$ & $-9,390$ \\
\hline Other & 5,262 & 3,771 & 6,071 & 5,275 & 4,948 \\
\hline Base Money & 23,172 & 24,930 & 26,909 & 28,392 & 31,027 \\
\hline Currency in circulation & 11,159 & 11,570 & 12,531 & 13,030 & 14,268 \\
\hline Commercial bank gourde deposits & 12,013 & 13,359 & 14,378 & 15,362 & 16,759 \\
\hline II. & ted Banki & System & & & \\
\hline Net foreign assets & 23,617 & 28,106 & 30,540 & 39,111 & 37,297 \\
\hline (In millions of U.S. dollars) & 604 & 773 & 804 & 979 & 881 \\
\hline Of which: Commercial banks NFA & 326 & 309 & 330 & 452 & 402 \\
\hline Net domestic assets & 51,474 & 50,557 & 56,469 & 53,469 & 66,057 \\
\hline Credit to the nonfinancial public sector & 20,248 & 18,852 & 18,852 & 13,224 & 19,603 \\
\hline Credit to the private sector & 27,019 & 29,946 & 33,639 & 37,496 & 43,274 \\
\hline In gourdes & 12,920 & 13,284 & 14,458 & 16,117 & 18,325 \\
\hline In foreign currency & 14,099 & 16,663 & 19,181 & 21,380 & 24,949 \\
\hline In millions of U.S. dollars & 360 & 458 & 505 & 535 & 589 \\
\hline Other & 4,207 & 1,760 & 3,979 & 2,748 & 3,179 \\
\hline Broad money & 75,091 & 78,664 & 87,009 & 92,580 & 103,355 \\
\hline Currency in circulation & 11,159 & 11,570 & 12,531 & 13,030 & 14,268 \\
\hline Gourde deposits & 31,533 & 32,974 & 35,940 & 37,050 & 41,496 \\
\hline Foreign currency deposits & 32,399 & 34,120 & 38,539 & 42,500 & 47,591 \\
\hline In millions of U.S. dollars & 828 & 938 & 1,014 & 1,064 & 1,124 \\
\hline & ercentag & hange) & & & \\
\hline Currency in circulation & 5.8 & 3.7 & 8.3 & 12.6 & 9.5 \\
\hline Base money & 5.5 & 7.6 & 7.9 & 13.9 & 9.3 \\
\hline Gourde money (M2) & 9.9 & 4.3 & 8.8 & 12.4 & 11.3 \\
\hline Broad money (M3) & 10.0 & 4.8 & 10.6 & 17.7 & 11.6 \\
\hline Gourde deposits & 11.5 & 4.6 & 9.0 & 12.4 & 12.0 \\
\hline Foreign currency deposits & 10.0 & 5.3 & 13.0 & 24.6 & 12.0 \\
\hline Credit to the nonfinancial public sector & -4.9 & -6.9 & 0.0 & -29.8 & 48.2 \\
\hline Credit to the private sector & 5.5 & 10.8 & 12.3 & 25.2 & 15.4 \\
\hline Credit in gourdes & -0.6 & 2.8 & 8.8 & 21.3 & 13.7 \\
\hline Credit in foreign currency & 11.8 & 18.2 & 15.1 & 28.3 & 16.7 \\
\hline Memorandum items: & & & & & \\
\hline Foreign currency bank deposits (percent of total) 2/ & 50.7 & 50.9 & 51.7 & 53.4 & 53.4 \\
\hline Foreign curr. credit to priv. sector (percent of total) & 52.2 & 55.6 & 57.0 & 57.0 & 57.7 \\
\hline Commercial Banks' Credit to Private Sector (\% GDP) & 12.9 & 12.4 & -- & 13.1 & 13.3 \\
\hline
\end{tabular}

Sources: Bank of the Republic of Haiti; and Fund staff estimates.

1/ Excluding commercial bank forex deposits, letters of credit, guarantees, earmarked project accounts and US\$-denominated bank reserves.

2/ Excluding public sector deposits. 
Table 4. Haiti: Balance of Payments

(Fiscal year ending September 30; in millions of US\$)

\begin{tabular}{|c|c|c|c|c|c|c|}
\hline & \multirow[b]{2}{*}{2006} & \multirow[b]{2}{*}{$2007^{-}$} & \multicolumn{2}{|l|}{2008} & \multicolumn{2}{|l|}{2009} \\
\hline & & & Prog. (Third PRGF Review) & Prel. & Prog. (Third PRGF Review) & Prog. \\
\hline Current account & -69.0 & -15.7 & -208.5 & -184.6 & -213.9 & -334.6 \\
\hline Current account (excluding grants) & -449.0 & -406.6 & -696.2 & -611.2 & -582.9 & -904.6 \\
\hline Trade balance & $-1,053.8$ & $-1,096.0$ & $-1,519.7$ & $-1,617.0$ & $-1,484.6$ & $-1,643.3$ \\
\hline Exports of goods & 494.4 & 522.5 & 490.9 & 490.2 & 588.8 & 498.9 \\
\hline Of which: Assembly industry & 435.0 & 463.1 & 430.7 & 423.3 & 525.7 & 438.0 \\
\hline Imports of goods & $-1,548.3$ & $-1,618.4$ & $-2,010.6$ & $-2,107.2$ & $-2,073.4$ & $-2,142.2$ \\
\hline Of which: Petroleum products & -397.1 & -415.0 & -655.8 & -602.2 & -702.3 & -426.4 \\
\hline Services (net) & -384.3 & -443.6 & -552.2 & -385.3 & -542.5 & -474.1 \\
\hline Receipts & 203.3 & 259.6 & 227.7 & 349.2 & 246.7 & 304.5 \\
\hline Payments & -587.6 & -703.2 & -779.9 & -734.5 & -789.2 & -778.5 \\
\hline Income (net) & 1.8 & 7.3 & 14.4 & 21.3 & 8.4 & -2.5 \\
\hline Of which: Interest payments & -16.9 & -19.6 & -19.7 & -24.6 & -18.7 & -26.2 \\
\hline Current transfers (net) & $1,367.4$ & $1,516.6$ & $1,849.1$ & $1,796.3$ & $1,804.9$ & $1,785.3$ \\
\hline Official transfers (net) & 380.0 & 390.9 & 487.7 & 426.6 & 369.0 & 570.0 \\
\hline Private transfers (net) & 987.4 & $1,125.7$ & $1,361.3$ & $1,369.7$ & $1,435.9$ & $1,215.3$ \\
\hline Capital and financial accounts & 148.0 & 179.1 & 158.5 & 226.1 & 73.7 & 176.2 \\
\hline Public sector capital flows (net) & 49.3 & 46.1 & 152.6 & 319.7 & 41.4 & 34.2 \\
\hline Loan disbursements & 84.5 & 91.6 & 200.7 & 363.5 & 87.9 & 85.5 \\
\hline Amortization & -35.3 & -45.5 & -48.1 & -43.8 & -46.4 & -51.3 \\
\hline Banks (net) $1 /$ & -80.2 & 16.2 & -20.6 & -143.0 & -28.1 & 50.0 \\
\hline Private sector capital flows $1 /$ & 163.0 & 73.0 & 26.4 & 27.9 & 60.4 & 92.0 \\
\hline Of which: Foreign direct investment & 160.0 & 74.5 & 26.4 & 27.9 & 60.4 & 20.0 \\
\hline Errors and omissions $2 /$ & 16.0 & 43.8 & 0.0 & 21.6 & 0.0 & 0.0 \\
\hline Overall balance & 79.1 & 163.4 & -49.9 & 41.5 & -140.2 & -158.3 \\
\hline Financing & -79.1 & -163.4 & 49.9 & -41.5 & -2.2 & 158.3 \\
\hline Change in net foreign assets $3 /$ & -87.6 & -184.3 & -10.6 & -63.4 & -32.9 & 48.2 \\
\hline Change in gross reserves & -109.3 & -207.9 & -33.4 & -163.0 & -50.6 & 49.0 \\
\hline Liabilities & 21.6 & 23.5 & 22.9 & 99.6 & 17.8 & -2.8 \\
\hline Utilization of Fund credits (net) & 10.6 & 21.0 & 22.9 & 49.9 & 22.9 & 23.3 \\
\hline Purchases and loans & 15.1 & 54.7 & 22.9 & 49.9 & 22.9 & 23.3 \\
\hline Repayments & -4.5 & -33.7 & 0.0 & 0.0 & 0.0 & 0.0 \\
\hline Other liabilities & 11.0 & 2.5 & 0.0 & 49.7 & -5.2 & -26.2 \\
\hline Change in arrears & 8.6 & -45.0 & 0.0 & 0.0 & 0.0 & 0.0 \\
\hline Debt rescheduling & 0.0 & 37.9 & 4.2 & 3.6 & 7.9 & 3.9 \\
\hline HIPC interim assistance & 0.0 & 28.1 & 19.7 & 18.3 & 22.8 & 19.1 \\
\hline External financing to be committed & 0.0 & 0.0 & 10.0 & 0.0 & 0.0 & 50.0 \\
\hline Prospective PRGF augmentation & 0.0 & 0.0 & 26.6 & 0.0 & 0.0 & 37.0 \\
\hline Financing gap & 0.0 & 0.0 & 0.0 & 0.0 & 142.4 & 0.0 \\
\hline \multicolumn{7}{|l|}{ Memorandum items: } \\
\hline Current account balance (in percent of GDP) & -1.4 & -0.3 & -3.0 & -2.6 & -2.9 & -4.4 \\
\hline Current account balance, excl. grants (in percent of GDP) & -9.3 & -6.6 & -10.0 & -8.6 & -7.9 & -11.9 \\
\hline Goods exports (f.o.b) growth & 7.7 & 5.7 & -6.0 & -6.2 & 19.9 & 1.8 \\
\hline Goods import (f.o.b) growth & 18.3 & 4.5 & 24.3 & 30.2 & 3.1 & 1.7 \\
\hline External debt as percent of exports & 203.4 & 197.1 & 228.5 & 225.7 & 206.1 & 241.0 \\
\hline Debt service as percent of exports & 7.5 & 8.3 & 9.4 & 8.2 & 7.8 & 9.6 \\
\hline Gross liquid international reserves (in millions of U.S. dollars & 337.1 & 544.7 & 578.1 & 707.8 & 628.8 & 696.0 \\
\hline Gross liquid international reserves (in months & & & & & & \\
\hline of next year's imports of goods and services) & 1.7 & 2.3 & 2.4 & 2.9 & 2.5 & 2.8 \\
\hline
\end{tabular}

Sources: Bank of the Republic of Haiti; and Fund staff estimates.

1/ For 2009, it assumes Petrocaribe-related resources constitute debt of a binational (Haiti-Venezuela) corporation.

2/ Includes short-term capital and errors and omissions for historical period.

$3 /$ Includes NIR and commercial banks' foreign currency deposits with the BRH.

4/ Gross liquid international reserves for FY2009 assume the disbursement of the proposed US $\$ 37$ million PRGF augmentation. 
Table 5. Haiti: Financial Soundess Indicators of the Banking System (Fiscal year ending September 30; in percent unless otherwise indicated)

\begin{tabular}{|c|c|c|c|c|}
\hline & 2005 & 2006 & 2007 & $\begin{array}{l}\text { Prel. } \\
2008 \\
\end{array}$ \\
\hline \multicolumn{5}{|l|}{ Size and Growth } \\
\hline Total assets (in millions of Gourdes) & 65,811 & 72,519 & 79,764 & 100,302 \\
\hline Of which: central bank bonds & 5,527 & 7,684 & 9,008 & 9,397 \\
\hline Of which: total loans & 22,065 & 22,750 & 24,670 & 31,187 \\
\hline Total assets (in US\$ millions) 1/ & 1,751 & 1,929 & 2,192 & 2,510 \\
\hline Total Deposits (in millions of Gourdes) & 56,771 & 61,311 & 66,031 & 84,725 \\
\hline Net Profits (loss) (in millions of Gourdes) & 114.3 & 414.4 & 202.3 & 483.7 \\
\hline Credit/GDP & 12.3 & 10.2 & 10.9 & 10.8 \\
\hline Deposits/GDP & 33.8 & 30.6 & 29.2 & 34.6 \\
\hline Credit growth (net) from year before 2/ & 5.6 & 13.7 & 9.9 & 4.1 \\
\hline \multicolumn{5}{|l|}{ Capital adequacy } \\
\hline Regulatory capital to risk-weighted assets $3 /$ & 16.5 & 14.3 & 19.0 & 12.6 \\
\hline Capital (net worth) to assets & 5.0 & 5.3 & 7.0 & 6.1 \\
\hline \multicolumn{5}{|l|}{ Asset quality and composition } \\
\hline Loans (net) to assets & 31.5 & 28.2 & 28.3 & 29.1 \\
\hline NPLs to gross loans & 12.4 & 11.1 & 10.0 & 9.7 \\
\hline Provisions to gross loans & 6.1 & 9.9 & 8.5 & 6.4 \\
\hline Provisions to gross NPLs & 49.1 & 89.3 & 85.5 & 66.4 \\
\hline NPL less provisions to net worth & 42.2 & 7.0 & 6.4 & 15.6 \\
\hline \multicolumn{5}{|l|}{ Earnings and profitability (annualized) } \\
\hline Net Earnings/Assets (ROA) & 0.7 & 1.8 & 1.0 & 2.0 \\
\hline Net Earnings/Equity (ROE) & 12.8 & 34.2 & 14.7 & 30.9 \\
\hline Net interest income to gross interest income & 71.8 & 72.2 & 67.1 & 80.0 \\
\hline Operating expenses to net profits & 80.5 & 70.7 & 86.0 & 73.5 \\
\hline \multicolumn{5}{|l|}{ Efficiency } \\
\hline Interest rate spread in Gourdes 4/ & 11.9 & 11.7 & 10.2 & 12.4 \\
\hline Interest rate spread in US dollar 4/ & 11.0 & 7.8 & 8.9 & 10.7 \\
\hline \multicolumn{5}{|l|}{ Liquidity } \\
\hline Liquid assets to total assets $5 /$ & 43.6 & 45.3 & 46.5 & 35.4 \\
\hline Liquid assets to deposits 5 / & 50.5 & 54.5 & 56.1 & 41.9 \\
\hline \multicolumn{5}{|l|}{ Market Risk } \\
\hline Foreign currency loans to total loans (net) & 59.3 & 66.0 & 70.1 & 69.3 \\
\hline Foreign currency deposits to total deposits 6/ & 52.6 & 53.6 & 52.4 & 58.2 \\
\hline
\end{tabular}

Source: Fund staff computation based on data from the Bank of the Republic of Haiti.

1/ Data for all years converted from gourdes.

2/ Net credit equal to gross loans less non performing loans.

$3 /$ The prudential requirement is 12 percent.

4/ Defined as the difference between average lending rate and average fixed deposit rate in the banking system.

5 / Liquid assets include cash and central bank bonds.

6/ Including public sector deposits. 
Table 6. Haiti: Indicative Targets and Quantitative Performance Criteria, FY 2008

\begin{tabular}{|c|c|c|c|c|c|c|c|c|c|}
\hline & \multirow[b]{3}{*}{$\begin{array}{l}\text { Actual Stock } \\
\text { at end-Sep. } \\
2007\end{array}$} & \multicolumn{8}{|c|}{ Cummulative Flows since 2007} \\
\hline & & \multicolumn{4}{|c|}{ Jun. 2008} & \multicolumn{4}{|c|}{ Sep. 2008} \\
\hline & & $\begin{array}{l}\text { Indicative } \\
\text { target }\end{array}$ & $\begin{array}{l}\text { Prog. with } \\
\text { adjustor }\end{array}$ & Actual & $\begin{array}{l}\text { Deviation } \\
\text { from prog } \\
\text { w/adjustor }\end{array}$ & $\begin{array}{l}\text { Test } \\
\text { date }\end{array}$ & $\begin{array}{l}\text { Prog. with } \\
\text { adjustor }\end{array}$ & $\begin{array}{l}\text { Prel. } \\
\text { actual }\end{array}$ & $\begin{array}{l}\text { Deviation } \\
\text { from prog } \\
\text { w/adjustor }\end{array}$ \\
\hline \multicolumn{10}{|l|}{ Performance criteria } \\
\hline Net central bank credit to the NFPS (in millions of gourdes) & 20,063 & 576 & 1,426 & 853 & -573 & 150 & 150 & 30 & -120 \\
\hline Central Government & 20,509 & 426 & 1,276 & 692 & -584 & 0 & 0 & -349 & -349 \\
\hline Rest of NFPS 1/ & -445 & 150 & 150 & 161 & 11 & 150 & 150 & 379 & 229 \\
\hline Net domestic banking sector credit to the central government $2 /$ & 19,588 & 426 & 426 & $-4,111$ & $-4,537$ & 0 & 0 & $-6,700$ & $-6,700$ \\
\hline Net domestic assets of the central bank (in millions of gourdes) - ceiling $3 /$ & 15,221 & 1,307 & 2,157 & 3,845 & 1,688 & 2,511 & 2,511 & 1,989 & -522 \\
\hline Domestic arrears accumulation of the central government $4 /$ & 0 & 0 & 0 & 0 & 0 & 0 & 0 & 0 & 0 \\
\hline $\begin{array}{l}\text { New contracting or guaranteeing by the central } \\
\text { government or the BRH of nonconcessional external debt 4/ 5/ } \\
\text { (In millions of U.S. dollars) }\end{array}$ & & & & & & & & & \\
\hline Up to and including one year & 0 & 0 & 0 & 0 & 0 & 0 & 0 & 0 & 0 \\
\hline Over one-year maturity & 0 & 0 & 0 & 0 & 0 & 0 & 0 & 0 & 0 \\
\hline Net international reserves of central bank (in millions of U.S. dollars) - floor $5 /$ & 270 & 0 & -22 & 26 & 48 & -14 & -14 & 25 & 39 \\
\hline External arrears accumulation (in millions of U.S. dollars) 4/ & 0 & 0 & 0 & 0 & 0 & 0 & 0 & 0 & 0 \\
\hline \multicolumn{10}{|l|}{ Indicative target: } \\
\hline Change in base money & 24,930 & 1,307 & 1,307 & 5,360 & 4,053 & 1,979 & 1,979 & 3,462 & 1,483 \\
\hline \multicolumn{10}{|l|}{ Memorandum items: } \\
\hline Change in currency in circulation & 11,570 & 377 & 377 & 995 & 618 & 960 & 960 & 1,460 & 500 \\
\hline Net domestic banking sector credit to the rest of the NFPS $5 /$ & -715 & -90 & -90 & 102 & 192 & 0 & 0 & 603 & 603 \\
\hline Government total revenue, excl. grants (in millions of gourdes) & $\ldots$ & 20,851 & 20,851 & 20,037 & -814 & 28,146 & 28,146 & 26,849 & $-1,297$ \\
\hline Government total expenditure, excl. ext-fin investment (in millions of gourdes) & & 24,026 & 24,026 & 24,427 & 401 & 32,639 & 32,639 & 32,308 & -330 \\
\hline
\end{tabular}

Sources: Ministry of Finance, Central Bank of Haiti, and Fund staff estimates.

1/ Includes non-budgeted autonomous organizations, local governments, and public enterprises.

2/ The observed decrease is explained by the accumulation, beginning in Q3, of Petrocaribe-related inflows in government deposits at commercial banks.

$3 /$ For program monitoring purposes, NDA is defined as monetary base minus Program NIR in gourde terms. Program exchange rate of G36/\$ through end-March,

and of $\mathrm{G} 38 / \$$ through end-September.

4/ On a continuous basis.

5/ Excludes letters of credit and guarantees, and earmarked projects. 
Table 7. Haiti: Structural Performance Criteria and Benchmarks for the Fourth Program Review

\begin{tabular}{|c|c|c|}
\hline Measures & $\begin{array}{c}\text { Date } \\
\text { (Month-end) }\end{array}$ & Status \\
\hline $\begin{array}{l}\text { 1. Structural performance criteria } \\
\text { - Limit current account spending to no more than } 10 \text { percent of } \\
\text { non-wage current expenditure. }\end{array}$ & Quarterly & Met \\
\hline $\begin{array}{l}\text { Prepare a strengthened plan to recapitalize the central bank. } \\
\text { - Complete independent assessment of an additional systemically } \\
\text { important bank. }\end{array}$ & $\begin{array}{l}\text { September } 2008 \\
\text { September } 2008\end{array}$ & $\begin{array}{l}\text { Met } \\
\text { Met }\end{array}$ \\
\hline 2. Structural benchmarks & & \\
\hline - Establish three new customs control posts on major roads. & September 2008 & Met in December \\
\hline $\begin{array}{l}\text { Develop and implement modules on the investment program for } \\
\text { the public financial management system SYSDEP. }\end{array}$ & September 2008 & Met in December \\
\hline $\begin{array}{l}\text { Develop and begin implementation of a plan to improve systemic } \\
\text { liquidity forecasting. }\end{array}$ & September 2008 & Met in January 2009 \\
\hline $\begin{array}{l}\text { - Initiate regular central bank reporting on monetary policy goals } \\
\text { and implementation. }\end{array}$ & September 2008 & Reset for end-March \\
\hline - Improve regulatory framework and supervision of credit unions. & September 2008 & Met \\
\hline - Submit a new organic law for the DGI to Parliament. & September 2008 & Reset for end-March \\
\hline
\end{tabular}


Table 8. Haiti: Proposed Schedule of Disbursements

\begin{tabular}{|c|c|c|}
\hline Amount & Date & Conditions for Disbursement $1 /$ \\
\hline SDR $28,100,000$ & November 20, 2006 & $\begin{array}{l}\text { Executive Board approval of the three-year arrangement } \\
\text { under the PRGF. Includes } 25 \% \text { of quota in access } \\
\text { for repayment of EPCA purchases }\end{array}$ \\
\hline SDR 7,600,000 & July 23, 2007 & $\begin{array}{l}\text { Observance of performance criteria for March } 2007 \text { and } \\
\text { completion of the first review under the PRGF arrangement. }\end{array}$ \\
\hline SDR $7,600,000$ & February 20, 2008 & $\begin{array}{l}\text { Observance of performance criteria for September } 2007 \text { and } \\
\text { completion of the second review under the PRGF arrangement. }\end{array}$ \\
\hline SDR $23,980,000$ & June 23, 2008 & $\begin{array}{l}\text { Observance of performance criteria for March } 2008 \text { and } \\
\text { completion of the third review under the PRGF arrangement. }\end{array}$ \\
\hline SDR 7,610,000 3/ & November 16, 2009 & $\begin{array}{l}\text { Observance of performance criteria for September } 2009 \text { and } \\
\text { completion of the sixth review under the PRGF arrangement. }\end{array}$ \\
\hline
\end{tabular}

1/ Other than the generally applicable conditions for the Poverty Reduction and Growth Facility (PRGF).

2/ At the time of the fourth review, an augmentation of 30 percent of the quota (SDR 24.57 million) is

proposed for the Board's consideration. This table incorporates the proposed phasing of the augmentation

(20 percent of quota to be disbursed upon completion of the fourth review, and 10 percent of quota to be

disbursed at the time of completion of the fifth review).

3/ An extension of the program beyond its expiration date (November 19, 2009) could be considered if additional time is needed to complete the sixth review. 
Table 9. Haiti: Indicators of Capacity to Repay the Fund, 2007-20 (Fiscal year ending September 30 )

\begin{tabular}{|c|c|c|c|c|c|c|c|c|c|c|c|c|c|c|}
\hline \multirow{3}{*}{$\overline{\text { Fund obligations based on existing credit }}$} & \multirow[b]{2}{*}{2007} & \multicolumn{13}{|c|}{ Projections } \\
\hline & & 2008 & 2009 & 2010 & 2011 & 2012 & 2013 & 2014 & 2015 & 2016 & 2017 & 2018 & 2019 & 2020 \\
\hline & \\
\hline Principal 3/ & 1.52 & 0.00 & 0.00 & 0.00 & 0.00 & 2.81 & 7.90 & 13.46 & 13.46 & 13.46 & 10.65 & 5.56 & 0.00 & 0.00 \\
\hline Charges and interest & 0.46 & 0.19 & 0.46 & 0.42 & 0.42 & 0.42 & 0.39 & 0.34 & 0.28 & 0.21 & 0.14 & 0.10 & 0.08 & 0.20 \\
\hline \multicolumn{15}{|c|}{$\begin{array}{l}\text { Fund obligations based on existing and prospective credit } 1 / \\
\text { (in millions of SDRs) }\end{array}$} \\
\hline Principal & 1.52 & 0.00 & 0.00 & 0.00 & 0.00 & 2.81 & 7.90 & 15.86 & 22.18 & 22.94 & 20.13 & 15.04 & 7.08 & 0.72 \\
\hline Charges and interest & 0.46 & 0.19 & 0.52 & 0.65 & 0.66 & 0.66 & 0.63 & 0.58 & 0.49 & 0.38 & 0.27 & 0.17 & 0.11 & 0.20 \\
\hline \multicolumn{15}{|l|}{ Total obligations based on existing and prospective credit $1 /$} \\
\hline In millions of SDRs & 1.97 & 0.19 & 0.52 & 0.65 & 0.66 & 3.47 & 8.53 & 16.44 & 22.67 & 23.32 & 20.40 & 15.21 & 7.19 & 0.92 \\
\hline In millions of U.S. dollars & 3.03 & 0.30 & 0.80 & 1.00 & 1.02 & 5.38 & 13.27 & 25.65 & 35.37 & 36.39 & 31.83 & 23.73 & 11.22 & 1.44 \\
\hline In percent of exports of goods and services & 0.39 & 0.04 & 0.12 & 0.14 & 0.12 & 0.53 & 1.21 & 2.18 & 2.81 & 2.70 & 2.20 & 1.52 & 0.67 & 0.08 \\
\hline In percent of debt service 2 I & 3.29 & 0.43 & 1.14 & 1.07 & 1.06 & 4.98 & 11.55 & 18.85 & 24.27 & 24.35 & 21.33 & 16.18 & 8.19 & 1.01 \\
\hline In percent of government domestic revenues & 0.49 & 0.04 & 0.10 & 0.11 & 0.10 & 0.48 & 1.08 & 1.94 & 2.50 & 2.41 & 1.96 & 1.35 & 0.60 & 0.07 \\
\hline In percent of quota & 2.41 & 0.23 & 0.63 & 0.79 & 0.81 & 4.24 & 10.42 & 20.07 & 27.68 & 28.47 & 24.91 & 18.57 & 8.78 & 1.12 \\
\hline In percent of gross international reserves & 0.51 & 0.04 & 0.11 & 0.13 & 0.13 & 0.63 & 1.49 & 2.76 & 3.63 & 3.56 & 2.97 & 2.11 & 0.95 & 0.12 \\
\hline \multicolumn{15}{|l|}{ Outstanding Fund credit $1 /$} \\
\hline In millions of SDRs & 35.7 & 67.3 & 107.1 & 114.7 & 114.7 & 111.9 & 104.0 & 88.1 & 65.9 & 43.0 & 22.8 & 7.8 & 0.7 & 0.0 \\
\hline In millions of U.S. dollars & 55.6 & 104.8 & 160.8 & 172.8 & 173.4 & 169.7 & 158.3 & 134.6 & 100.7 & 65.6 & 34.9 & 11.9 & 1.1 & 0.0 \\
\hline In percent of exports of goods and services & 7.1 & 14.8 & 23.7 & 23.5 & 20.1 & 16.9 & 14.4 & 11.4 & 8.0 & 4.9 & 2.4 & 0.8 & 0.1 & 0.0 \\
\hline In percent of debt service 2 I & 60.4 & 153.0 & 229.9 & 184.5 & 179.5 & 157.2 & 137.9 & 98.9 & 69.1 & 43.9 & 23.4 & 8.1 & 0.8 & 0.0 \\
\hline In percent of government domestic revenues & 9.0 & 14.9 & 20.0 & 19.1 & 17.3 & 15.3 & 12.8 & 10.2 & 7.1 & 4.3 & 2.1 & 0.7 & 0.1 & 0.0 \\
\hline In percent of quota & 43.6 & 82.1 & 130.7 & 140.0 & 140.0 & 136.6 & 126.9 & 107.6 & 80.5 & 52.5 & 27.9 & 9.5 & 0.9 & 0.0 \\
\hline In percent of gross international reserves & 9.3 & 13.8 & 22.6 & 22.4 & 21.4 & 20.0 & 17.8 & 14.5 & 10.3 & 6.4 & 3.3 & 1.1 & 0.1 & 0.0 \\
\hline \multicolumn{15}{|l|}{ Memorandum items: } \\
\hline Exports of goods and services (millions of U.S. dollars) & 782.1 & 706.3 & 677.9 & 734.9 & 863.8 & 1007.2 & 1100.8 & 1175.8 & 1258.1 & 1348.3 & 1447.4 & 1556.3 & 1676.2 & 1805.0 \\
\hline Debt service (millions of U.S. dollars) 2/ & 91.9 & 68.5 & 69.9 & 93.7 & 96.6 & 108.0 & 114.8 & 136.1 & 145.8 & 149.5 & 149.2 & 146.7 & 136.9 & 142.1 \\
\hline Domestic Revenues (millions of U.S. dollars) & 620.2 & 701.7 & 804.3 & 903.0 & 1003.3 & 1111.2 & 1232.8 & 1320.5 & 1416.6 & 1510.9 & 1626.1 & 1752.8 & 1879.2 & 2028.5 \\
\hline Quota (millions of SDRs) & 81.9 & 81.9 & 81.9 & 81.9 & 81.9 & 81.9 & 81.9 & 81.9 & 81.9 & 81.9 & 81.9 & 81.9 & 81.9 & 81.9 \\
\hline Gross international reserves (millions of U.S. dollars) & 595.9 & 758.9 & 709.9 & 772.3 & 809.1 & 847.7 & 888.2 & 930.7 & 975.4 & 1022.3 & 1071.6 & 1123.3 & 1177.6 & 1234.6 \\
\hline GDP (millions of U.S. dollars) & 6137.4 & 7108.3 & 7589.2 & 8040.6 & 8519.2 & 9042.0 & 9539.0 & 10015.9 & 10537.1 & 11106.7 & 11729.6 & 12411.3 & 13157.8 & 13949.1 \\
\hline
\end{tabular}

Sources: Haitian authorities; and Fund staff estimates and projections.

1/ Assumes augmentation of SDR 24.57 million proposed to the Board in February 2009 is disbursed during calendar year 2009 (20 percent of quota disbursed upon completion of fourth review, and 10 percent of quota disbursed at the time of completion of the fifth review.

2/ Net of interim HIPC assistance, including proposed increase in assistance of SDR 33,000.

3/ it does not include an SDR 20.5 million EPCA repurchase in December 2006 
Table 10. Haiti: Status of HIPC Completion Point Triggers (January 2009)

\begin{tabular}{|c|c|c|}
\hline Completion point trigger & Status & Expected fulfillment \\
\hline PRSP & Annual Progress Report covering one year of implementation required. & Realization expected. \\
\hline Macroeconomic stability & Performed well under PRGF-supported program despite multiple shocks. & Realization expected. \\
\hline \multicolumn{3}{|l|}{ PFM and Governance } \\
\hline $\begin{array}{l}\text { a. Tracking poverty-reducing spending and publishing quarterly } \\
\text { reports at least } 6 \text { months prior to CP. }\end{array}$ & $\begin{array}{l}\text { Poverty-reducing spending being tracked based on existing classifications. Quarterly } \\
\text { reports published through June } 2008 \text { and an FY } 2008 \text { table was provided to IMF mission. }\end{array}$ & Realization expected. \\
\hline b. Align public spending priorities with PRSP. & $\begin{array}{l}\text { Execution of FY08 budget and plans for FY } 2009 \text { will be used to evaluate first year of } \\
\text { implementation and will be reflected in the Annual Progress Report. }\end{array}$ & Realization expected. \\
\hline $\begin{array}{l}\text { c. Submit government account audits to the Court of Accounts and to } \\
\text { parliament, in conformity with legally mandated calendar. }\end{array}$ & $\begin{array}{l}\text { Court has issued comments, and the audit and opinion were submitted to Parliament } \\
\text { together with the FY } 2009 \text { budget. }\end{array}$ & \\
\hline $\begin{array}{l}\text { d. Adopt and implement a new law for public procurement, in line } \\
\text { with international best practice. Audits for all contracts over US } \$ 1 \mathrm{~m} \\
\text { and random audits of other contracts for six months prior to the CP. }\end{array}$ & The draft procurement law is scheduled to be submitted and ratified in January 2009. & $\begin{array}{l}\text { Once the law is submitted } \\
\text { and passed, six months } \\
\text { needed to conduct audits. }\end{array}$ \\
\hline $\begin{array}{l}\text { e. Adoption of a law on asset declaration and submission of one } \\
\text { annual compliance report on monitoring of asset declarations } \\
\text { covering the preceding year. }\end{array}$ & $\begin{array}{l}\text { Law in effect since February } 2008 \text { and asset declarations have been undertaken for } 3-4 \\
\text { months. A report on the first year since the law took effect will be submitted to the Court of } \\
\text { Accounts and Parliament in February } 2009 \text {. }\end{array}$ & Realization expected. \\
\hline \multicolumn{3}{|l|}{ Structural Reforms } \\
\hline $\begin{array}{l}\text { a. Reinforcing and establishing customs control in Cap Haitien, } \\
\text { Gonaives, St. Marc, Miragoane, Malpasse, Ouanaminthe and } \\
\text { Belladere, including by installing ASYCUDA/SYDONIA. }\end{array}$ & $\begin{array}{l}\text { SYDONIA World is operational in Port-au-Prince at the airport, port and St. Marc. It will be } \\
\text { extended to other locations (except hurricane-affected Gonaives and Miragoane) by } \\
\text { February } 2009 \text {. }\end{array}$ & Realization expected. \\
\hline $\begin{array}{l}\text { b. Extending use of central taxpayer file to all taxpayers in PaP and } \\
\text { registering all the taxpayers identified in the tax centers of Cayes, } \\
\text { Miragoane, St. Marc, Port de Paix, Cap Haitien, and Fort Liberte. }\end{array}$ & $\begin{array}{l}\text { The central taxpayer file is being used for all taxes throughout Port-au-Prince and has had } \\
\text { a large impact on tax revenues. Already being used for four largest taxes in the provinces. }\end{array}$ & Realized. \\
\hline \multicolumn{3}{|l|}{ Education } \\
\hline $\begin{array}{l}\text { a. Public financing mechanism to get } 50,000 \text { children into primary } \\
\text { school, verified by independent audit. }\end{array}$ & $\begin{array}{l}\text { More than } 50,000 \text { additional children have entered primary school although hurricanes } \\
\text { have kept some previous attendees out of school this year. The process for an } \\
\text { independent audit underway. }\end{array}$ & $\begin{array}{l}\text { Realization expected, audit } \\
\text { report due in June } 2009 .\end{array}$ \\
\hline $\begin{array}{l}\text { b. Spending for education reaches at least } 21 \% \text { of actual total } \\
\text { recurrent spending, of which } 50 \% \text { on primary education over the } \\
\text { year prior to CP and training of } 2,500 \text { new teachers and two visits } \\
\text { per year of all primary schools by inspectors. }\end{array}$ & $\begin{array}{l}\text { FY } 2008 \text { budget had education as } 20.3 \% \text { of recurrent spending, of which } 38 \% \text { on primary } \\
\text { school. Percentage could exceed } 50 \text { after additional spending for the "rentrée scolaire" in } \\
\text { September. Some } 2500 \text { teachers in training by September } 2008 \text {. The number of } \\
\text { inspectors has been doubled to } 600 \text {, and achievement of goal of two visits per school on } \\
\text { average is underway. }\end{array}$ & $\begin{array}{l}\text { Further review needed. } \\
\text { Realization on spending } \\
\text { targets expected. Training of } \\
\text { teachers and school visits } \\
\text { under way. }\end{array}$ \\
\hline \multicolumn{3}{|l|}{ Health } \\
\hline $\begin{array}{l}\text { a. Increase by } 10 \text { percentage points immunization rates for DPT3, } \\
\text { BCG and measles. }\end{array}$ & $\begin{array}{l}\text { Table provided to November } 2008 \text { mission shows achievement on DPT3 and measles, } \\
\text { but less progress on BCG (rate already high on BCG). }\end{array}$ & $\begin{array}{l}\text { Strict realization may not be } \\
\text { met by June } 2009 \text { for BCG. }\end{array}$ \\
\hline $\begin{array}{l}\text { b. Approval by Government of National Policy, Strategic Plan and } \\
\text { Scale Up Operational Plan for HIVIAIDS prevention and treatment. }\end{array}$ & PM approved the Strategic Plan and an integrated operational plan. & Realized. \\
\hline \multicolumn{3}{|l|}{ Debt management } \\
\hline $\begin{array}{l}\text { a. Centralize all info on public external and domestic foreign } \\
\text { currency denominated debt in a single database. }\end{array}$ & $\begin{array}{l}\text { SYGADE software for centralized database is ready. Hardware is currently under } \\
\text { installation in the Ministry of Economy and Finance. UNCTAD consultant is currently } \\
\text { providing technical assistance for the use of the software SYGADE. }\end{array}$ & Realization expected \\
\hline $\begin{array}{l}\text { b. Publication of two quarterly reports on external debt data prior to } \\
\text { CP. }\end{array}$ & $\begin{array}{l}\text { Once centralized database has been established, reports can be generated to cover end- } \\
\text { September } 2008 \text { and end-December } 2008 \text {. }\end{array}$ & Realization expected. \\
\hline
\end{tabular}


Table 11. Haiti: Indicators of External Vulnerability 2007-09

(Units as indicated)

\begin{tabular}{lrrr}
\hline & & Prel. & Prog. \\
& 2007 & 2008 & 2009 \\
\hline Debt indicators & 29.1 & 26.7 & 25.5 \\
Total external public debt (in percent of GDP) 1/ & 197.0 & 225.7 & 241.0 \\
Total external public debt (in percent of exports 2/) & 1.1 & 1.0 & 1.0 \\
External debt service (in percent of GDP) & 0.7 & 0.6 & 0.7 \\
Amortization & 0.3 & 0.3 & 0.3 \\
Interest & 8.3 & 8.2 & 9.6 \\
External debt service (in percent of exports 2/) & 5.8 & 5.2 & 6.4 \\
Amortization & 2.5 & 2.9 & 3.3 \\
Interest & 10.2 & 10.2 & 10.0 \\
External debt service (in percent of current central govt. revenues) & 7.1 & 6.5 & 6.6 \\
Amortization & 3.1 & 3.7 & 3.4 \\
Interest & & & \\
Other indicators & & & \\
Exports (percent change, 12-month basis in U.S. dollars) & 5.7 & -6.2 & 1.8 \\
Imports (percent change, 12-month basis in U.S. dollars) & 4.5 & 30.2 & 1.7 \\
Remittances and grants in percent of gross disposable income & 19.8 & 20.1 & 19.0 \\
Real effective exchange rate appreciation (+) (end of period) & 14.9 & $\ldots$ & $\ldots$ \\
Exchange rate (per U.S. dollar, period average) & 37.4 & $\ldots$ & $\ldots$ \\
Current account balance (US million) 3/ & -15.7 & -184.6 & -334.6 \\
Capital and financial account balance (US\$ million) 4/ & 179.1 & 226.1 & 176.2 \\
Public sector & 46.1 & 319.7 & 34.2 \\
Private sector & 133.0 & -93.6 & 142.0 \\
Liquid gross reserves (US \$ million) 5/ & 544.7 & 707.8 & 659.0 \\
In months of imports of the following year 2/ & 2.3 & 2.9 & 2.6 \\
In percent of debt service due in the following year & 796 & 913 & 944 \\
In percent of base money & 79.5 & 99.6 & 90.0 \\
\hline
\end{tabular}

Sources: Bank of the Republic of Haiti; and Fund staff estimates.

$1 /$ It does not reflect completion point debt reduction in 2009.

2/ Goods and services.

$3 /$ Including grants.

4/ Includes in the private sector FDI, short-term capital, and errors and omissions in addition to bank flows.

5/ For FY 2009, it excludes the US\$ 37 million proposed PRGF augmentation. 


\section{ATTACHMENT I. HAITI: LETTER OF INTENT}

Mr. Dominique Strauss-Kahn

Managing Director

International Monetary Fund

700 19th Street, N.W.

Washington, DC 20431

U.S.A.
Port-au-Prince

February $2^{\text {nd }}, 2009$

Dear Mr. Strauss-Kahn:

1. The purpose of this letter and attached memorandum is to inform you of the progress made under the PRGF-supported program, and to request that the fifth disbursement under the arrangement, in the amount of SDR 7.6 million, be made available to Haiti following the completion of the fourth review. In addition, given the damage caused by recent natural disasters and its expected negative impact on Haiti's external position, we are requesting an augmentation of access under the arrangement of SDR 24.57 million (30 percent of quota). Of this total amount, SDR 16.38 million (20 percent of quota) are to be made available following completion of the fourth review and SDR 8.19 million upon completion of the fifth review.

2. The attached Supplementary Memorandum of Economic and Financial Policies (MEFP) reviews progress under the PRGF and outlines the government's polices, objectives, and macroeconomic framework for FY 2008-09 (October 2008-September 2009). Haiti's performance during the first two years of the PRGF-supported program has been strong. We have carried out a reform program that has improved fiscal discipline, expenditure execution, and transparency, as well as strengthened the banking system. This has contributed to stabilizing the economy, improving private sector confidence, and accelerating economic growth, though slower than anticipated.

3. For the fourth review, all but one quantitative performance criteria (PC), all three structural PCs, and one of six structural benchmarks were fully implemented prior to the endSeptember 2008 test date. The Government requests a waiver for the nonobservance of the end-September quantitative PC on central bank credit to the non-financial public sector, which was missed by a small margin (less than 0.1 percent of GDP). Three of the five remaining end-September structural benchmarks were completed by end-January, and we request that the other two benchmarks be reset for end-March 2009.

4. During the third program year, despite the serious difficulties that we are facing because of recent shocks, we will raise domestic revenue through the implementation of new tax measures and strengthened fiscal revenue administration to support higher public investment and poverty-reducing expenditures, reduce inflation, and promote private sectorled growth and job creation. 
5. Four back-to-back hurricanes and tropical storms in August and September caused flooding and mudslides, which devastated the country's infrastructure and wiped out about 60 percent of the summer-fall food harvest. The damage caused by the natural disasters has been estimated at about 15 percent of GDP. Substantial imports of staples and construction services will be necessary to rebuild infrastructure, which would lead to a deterioration of our external position in spite of the recent fall in commodity prices. Moreover, private remittances, which support domestic private consumption, have begun to decelerate since the onset of the sub-prime crisis in the U.S. We anticipate that remittances will continue to decline with the economic downturn in the U.S. and Canada, as will demand in our main export markets, including for tourism services. The government has decided to use saved PetroCaribe resources to address part of the initial emergency spending for reconstruction and other needs, and is working to strengthen revenue administration. We have also received additional support from our development partners, but much more is needed to address the difficult humanitarian situation and to rebuild and develop our country.

6. To help smooth the needed adjustment to the deteriorating external position, we are requesting additional access under the PRGF arrangement. We understand that it is unusual to request two consecutive program augmentations and that this would bring us to the maximum normal PRGF access level. ${ }^{1}$ This request is justified by the dire circumstances and the multiple exogenous shocks, and is consistent with our effort to scale back our spending plans and generate new domestic revenues, as well as with additional commitments being made by other multilaterals. Moreover, a strong signal by the Fund is expected to help catalyze additional support from our other development partners.

7. The Government remains committed to implementing the Poverty Reduction Strategy Paper (PRSP), but its implementation will be affected by the challenges arising from recent shocks. We are also working on the remaining outstanding structural measures needed to achieve the floating completion point for HIPC, which we hope to reach in mid-2009.

8. The Government believes that the policies set forth in the attached MEFP are adequate to achieve the objectives of its program, but it will take any further measures that may become appropriate for this purpose. Haiti will consult with the Fund on the adoption of these measures, and in advance of any revision to the policies contained in the MEFP, in accordance with the Fund's policies on such consultations.

\footnotetext{
${ }^{1}$ As a large importer of food and fuel, our external position deteriorated significantly earlier in the year, and we requested a PRGF augmentation at the time of the third review.
} 
9. In line with our demonstrated commitment to transparency, we agree to the publication of the staff report for the fourth review under the PRGF arrangement.

Sincerely yours,

/s/

Daniel Dorsainvil

Minister of Economy and Finance

Haiti
$/ \mathrm{s} /$

Charles Castel

Governor

Bank of the Republic of Haiti

Attachments 


\section{AtTachment II. Haiti: Memorandum on Economic and Financial Policies FOR FISCAL YEAR 2009}

1. Haiti has recently experienced a series of devastating shocks that have fueled inflation and hindered economic growth. Rising food and fuel prices prompted civil unrest and a political stalemate that severely constrained government operations. In addition, a series of tropical storms and hurricanes in August and September caused a substantial loss of lives and extensive damage to homes, agricultural production, and the country's infrastructure.

Estimates by the UN/EU/World Bank put the economic damage at as much as 15 percent of GDP. In addition, the downturn in the U.S. and Canadian economies triggered by the global financial crisis has caused a deceleration in remittances inflows that is expected to intensify and continue for several months.

2. Despite these shocks, we were able to comply with most of our commitments under the PRGF-supported program and maintain fiscal and monetary discipline. The impact of the shocks will endure through the current fiscal year and possibly beyond, and substantial resources will be required to cover humanitarian and reconstruction needs in addition to the financing required to implement the PRSP development strategy. Although the shocks have shaken some of the advances made in the past four years, we will continue to make the utmost effort to maintain macroeconomic stability in the current challenging environment. We are determined to continue working with our development partners to restore infrastructure damaged by the hurricanes and to deepen implementation of our home-grown development strategy, as laid out in the PRSP. This strategy aims to put Haiti in a position to generate sustainable, job-creating growth that will be conducive to poverty reduction and a lasting improvement in the living conditions of our population.

3. This Memorandum of Economic and Financial Policies (MEFP) reviews the progress under the PRGF-supported program and outlines the government's policies, objectives, and macroframework for the third and final year of the PRGF program covering FY 2009 (October 2008-September 2009).

\section{Recent Developments}

4. Program objectives, particularly inflation, were amended at the time of the third review to reflect increased food and fuel prices in the first half of the fiscal year. However, continued high international food prices, supply side effects of the hurricanes, and annual gourde depreciation of close to 10 percent caused 12-month inflation to peak at 19.8 percent in September 2008, compared to 7.9 percent a year earlier. After declining slightly in October, annual inflation fell to 10.1 percent in December, reflecting sharp decreases in international food and fuel prices.

5. Growth for FY 2008 is now estimated at 1.3 percent (lower than expected), compared to 3.4 percent in FY 2007. Decelerating remittances and the impact of the hurricanes had a negative impact on private consumption, while public spending provided some positive 
stimulus, due to improved budget execution capacity. The deficit in the balance of goods and services is estimated to have widened by US\$521 million (7.3 percent of GDP) in FY 2008, largely because of higher import bills for food and fuels.

6. The overall fiscal deficit (excluding grants and foreign-financed investment) in FY 2008 reached 2 percent of GDP, compared with 1.7 percent in the program, reflecting spending in line with projections (excluding foreign-financed investment) and revenues somewhat lower than projected. Domestically-financed capital expenditures exceeded program expectations as a result of improved budget execution procedures, as well as additional spending for public works and in support of agricultural production. Temporary subsidies were instated to respond to the social tension caused by the commodity price crisis, and these increases were partly offset by reductions in the wage bill and purchases of goods and services. Domestic revenues were 9.9 percent of GDP, compared with 10.6 percent programmed, with the shortfall reflecting mainly losses from the suspension of fuel price adjustments in early April 2008. ${ }^{1}$ However, the rice subsidy was phased out in August and the automatic fuel price adjustment mechanism was reinstated in mid-October.

7. Delays in donor disbursements resulted in significant reliance on temporary central bank (BRH) credit to the government during the second half of FY 2008, including to finance outlays for the start of the school year, while investment expenditures were curtailed to stay within reach of the adjusted end-September program ceiling. However, as the impact of the hurricanes required additional outlays, US\$51 million in accumulated PetroCaribe resources was transferred to the BRH (one of the components of net credit to the government), resulting in compliance with the program criterion to have no new BRH financing to the central government.

8. Our monetary policy was aimed at containing core inflation from second round effects derived from the commodity price shock and smoothing out the exchange rate path. In the context of higher headline inflation, we doubled the nominal interest rate for BRH bonds, we sterilized temporary central bank financing to the government through the placement of BRH bonds (G 1.5 billion) and net foreign exchange sales (US\$52.2 million). Despite these efforts, base money growth by end-September (14 percent) remained above its program target (7.9 percent), but still well below nominal GDP growth. With the objective of progressively extending the yield curve beyond 91 days, a new 182-day bond was introduced. Although participation in weekly central bank bond auctions was opened up to nonbank financial institutions, participation remains concentrated to a few large commercial banks.

\footnotetext{
${ }^{1}$ Real and nominal GDP for FY 2007 and FY 2008 have been revised since the program was agreed. When using the original program nominal GDP, tax revenue in FY 2008 was 10.3 percent of GDP, compared to 10.6 percent of GDP in the program.
} 
9. End-September data indicate that key quantitative performance criteria of the program, including zero central bank financing to the central government and the floor on NIR accrual, were met. The criterion on BRH credit to the rest of the nonfinancial public sector was breached by less than 0.1 percent of GDP.

10. The three end-September structural PCs were met. The MEF and BRH submitted a strengthened central bank recapitalization plan, audits were conducted on two other systemically-important commercial banks, and spending from current accounts was contained to well below 10 percent of non-wage current expenditures. We also made progress in the implementation of the six program benchmarks, although only one benchmark was completed by end-September (the BRH updated its prudential regulations and corresponding guidelines on the supervision of credit unions and prepared a draft law to improve the regulatory framework and supervision of credit unions). We request that two benchmarks be reset for end-March 2009: a new organic law for the DGI will be submitted to Parliament (the draft law has been approved by cabined and reviewed by the IMF's Fiscal Affairs Department); and we will start publishing regular reports on inflation and monetary policy. Regarding the latter, we have already stepped up our communication efforts on monetary policy goals and implementation through speeches and occasional press interviews. Two of the remaining three benchmarks were implemented by end-December: (i) the three customs posts are operational; and (ii) we are now recording investment spending in our financial management software (SYSDEP). We have improved the coordination between the central bank and the Ministry of Economy and Finance to strengthen systemic liquidity forecasting, and a formal plan to reinforce this was finalized in January.

\section{Program Objectives for FY 2009}

11. Our priorities for FY 2009, the final year of the PRGF-supported program, will be to increase spending for infrastructure rehabilitation, humanitarian needs and PRSP implementation, while maintaining the principle of sound financing. We are committed to continued adherence to the program to maintain stability, set the stage for recovery of the economy, and complete structural reforms initiated over the course of the three-year PRGFsupported program. We are also committed to implementing all remaining measures needed to reach the HIPC completion point in mid-2009. This will be a challenge given the deteriorating external environment and the need to recover from the severe external shocks.

\section{Growth and Inflation}

12. Growth in FY 2009 is projected at 2.5 percent, reflecting significant public sector spending on restoring infrastructure, including the beginning of operations of three new power plants. Negative supply-side effects of the hurricane damage to road infrastructure and the agricultural sector are likely to hinder a faster recovery. From the demand side, this reflects a reduction in private consumption from declining remittances, as well as lower FDI and net exports due to the economic downturn in the U.S. and Canada. Lower net exports 
also reflect slowing U.S. demand for Haiti's exports and a need to import basic food products, higher volumes of fuel to operate the new power plants, and goods and services for the reconstruction of infrastructure. In light of the worsening global environment, downside risks to the growth outlook are significant, notably through possibly even lower remittances and export demand than anticipated.

13. End-September 12-month inflation peaked at 19.8 percent and further declined to 10.1 percent at end-December. However, although international commodity prices are declining rapidly, rigidities such as supply shortages, long purchasing contracts, and price gouging, together with faster exchange rate depreciation may slow the impact on headline inflation, which is projected to remain relatively high at 9.5 percent by end-September 2009 . In light of weak demand from other sectors of the economy, higher fiscal spending to address hurricane damages is unlikely to contribute substantially to inflationary pressures, and we will ensure that any temporary central bank financing is limited and sterilized.

\section{Fiscal}

14. The fiscal program will seek to balance large spending needs for emergency, reconstruction and PRSP implementation with sound financing principles to maintain stability. As a result, fiscal policy in FY 2009 will be more expansionary than in previous years under the program.

15. The submission of the FY 2009 budget has been delayed, but a supplemental budget was approved at the end of FY 2008 that provides ongoing spending authority until a new budget can be submitted to Parliament. We have submitted the FY 2009 budget to Parliament on December 19, 2008 and expect its approval by end-January, 2009. The FY 2009 budget includes a modest increase in current spending: wages increase by 0.6 percentage points reflecting hirings of teachers and police, payment of a one-off $14^{\text {th }}$ salary to civil servants, granted in lieu of a general salary increase, and higher salaries for judges, and transfers increase by 0.4 percentage points to support increased electricity production, while other current spending is reduced by 0.3 percentage points of GDP. The budged also features an ambitious program of domestically-financed expenditures (an increase of 1.8 percentage points of GDP over FY 2008), that aims at stimulating growth, at increasing the availability of animal products through the promotion of rearing, fishing and aquaculture, at protecting the population against floods, at improving sanitary conditions, and improving formal and education supply, in line with the PRSP priorities. Almost 35 percent of domesticallyfinanced capital spending is allocated to the Ministry of Public Works, 5 percent to Health and Education, 4 percent to Agriculture, and 3 percent to Public Security.

16. The government is in the process of executing $\mathrm{G} 9.1$ billion in off-budget reconstruction spending (2.9 percent of GDP), authorized by Parliament in late September by passage of a law for emergency spending which, during a state of emergency, grants the government extended powers to reallocate budgetary funds, execute off-budget spending and 
award public contracts through expedited procedures. For the purposes of the PRGFsupported program, on-budget and off-budget spending will be consolidated. Total programmed expenditures for FY 2009, including foreign-financed investment, are projected at $\mathrm{G} 61.1$ billion.

17. Our program features an increase in domestic revenue to the equivalent of 10.5 percent of GDP. The projection reflects lower fuel prices and the resumption since midOctober 2008 of automatic fuel price adjustments, mitigated by: the impact of the new customs control posts established in major roads; the full-year impact of the new centralized taxpayer database; and continued implementation of the income tax law approved in FY 2007. In addition, ongoing and upcoming technical assistance from a number of donors will contribute to strengthened tax and customs administration in the months ahead. However, low growth and low oil prices will necessarily limit the overall amount that can be collected. Should revenue collection fall below our objectives, we are committed to reducing non-priority spending commitments accordingly. The total resource envelope, including budget support grants and project grants, is estimated at G 48.8 billion.

18. These estimates leave a projected overall deficit for FY 2009 (excluding project grants and foreign-financed investment) of G 8.8 billion (2.8 percent of GDP), including offbudget emergency spending. To finance the deficit and Haiti's debt service, we have so far identified new budget support of $\mathrm{G} 6$ billion, which mostly consists of accumulated PetroCaribe resources, as well as debt relief and debt rescheduling of G 0.9 billion, and a drawdown of $\mathrm{G} 0.3$ billion in accumulated government deposits at the BRH. In addition, we expect to mobilize additional donor support of at least $\mathrm{G} 2.0$ billion to cover our remaining financing needs, in part at the occasion of a donor conference during the first quarter of 2009. If this amount does not materialize, the program adjustor will allow new central bank financing of up to G 2 billion (US\$50 million) to cover the shortfall. If such central bank financing is needed, in keeping with our central program commitment, we will budget resources for its repayment in the following fiscal year.

19. The government's off-budget emergency spending program will be financed by use of saved PetroCaribe resources. We are committed to monitoring this off-budget spending very closely. We will record the execution of emergency spending using normal budget classification procedures, request detailed spending plans and reports from spending units, and submit to Parliament and publish detailed quarterly execution reports until all such spending is executed. Finally, all off-budget spending will be audited in line with normal audit procedures for on-budget spending.

20. For FY 2009, net external financing under the program does not include any new PetroCaribe/ALBA resources. In case they materialize, any new PetroCaribe resources that constitute public debt and/or are spent by the central government in FY 2009 will be treated 
as budget support or as project account resources for program purposes, as specified in the attached Technical Memorandum of Understanding ( $\uparrow \uparrow \mid 2,27-28$ ). ${ }^{2}$

21. We are committed to keeping up-to-date the detailed reporting table on transfers to the electricity company EdH, which was developed earlier on under the program. Close monitoring of these transfers is essential to ensuring continued fiscal soundness. This oversight will be complemented by ongoing efforts to improve the operational efficiency of $\mathrm{EdH}$, which are being undertaken with support from the World Bank and other donors. Improving the provision of electricity and bringing down energy costs are critical improvements needed to ensuring strengthened growth prospects for Haiti.

\section{Monetary and Financial Sector}

22. Monetary policy will remain geared to keeping core inflation reined-in, as the impact of high commodity prices wanes. To this end, the annual rate of base money growth will be kept at around 9.3 percent during FY 2009, or below nominal GDP growth. With this objective in mind, the BRH will sterilize temporary central bank financing through open market operations and foreign exchange sales. While the BRH may undertake foreign exchange operations to smooth out exchange rate adjustment, including in response to shocks, it remains committed to its flexible exchange rate regime, which will continue to be an important instrument to help the economy adjust to shocks. To help guide inflation expectations, we commit to publishing, beginning in early 2009 , regular reports on monetary policy goals and outcomes. We will ensure close coordination between the Ministry of Economy and Finance and the BRH to assess upcoming liquidity needs, including for foreign exchange. We will also continue to encourage broader participation in the weekly central bank bond auctions, as our medium-term objective remains to create a competitivelydetermined policy interest rate that can serve as a better transmission mechanism for monetary policy objectives.

23. Prudential indicators through end-September 2008 suggest that the banking sector has remained in broadly sound condition. Liquidity and capitalization remain solid. Net profits of the banking system doubled from a year ago, and non-performing loans declined below 10 percent. The assessments by independent experts of the BNC and the two other large banks in the system were finalized and are broadly satisfactory. The BNC will require an increase in its capital base to accommodate the absorption of Socabank in 2006, and a review of its operational structure, to which we are committed. The BRH will remain vigilant and monitor developments closely to ensure the continued health of the banking system. The $\mathrm{BRH}$ has drafted a new law to improve the supervision of credit unions and is working on

\footnotetext{
${ }^{2}$ The nature of these resources will be determined by their originating contract and the definitions in the attached Technical Memorandum of Understanding ( $₫ \uparrow 2,27-28)$.
} 
other key recommendations suggested by the IMF-World Bank financial sector assessment carried out in late 2007, and by the Fund's safeguards assessment.

\section{Quantitative Program Targets}

24. The third year of the program will be monitored using quarterly quantitative indicative targets and semi-annual quantitative PCs as presented in Table 2. The test dates for the program will be end-March and end-September 2009. It is anticipated that the fifth program review will be completed on or around June 29, 2009, and that the sixth review will be completed on, or around, November 16, 2009. With the exception of the drawing down of government deposits accumulated at end-September 2008 (G 349 million), new central bank financing to the non-financial public sector will be zero. The proposed program provides some room for BRH credit to the government in the first two quarters, which are likely to be the most critical from a humanitarian and reconstruction perspective, but this credit is programmed to be reversed by end-September 2009, in the absence of use of the program adjustor. To allow for some smoothing of the balance of payments adjustment, the program provides for a reduction in NIR of US\$50 million during FY 2009. To ensure that this will not reduce reserve coverage, we are requesting an augmentation of access under the program of 30 percent of quota (US\$37 million), of which 20 percent of quota would be disbursed upon completion of the fourth review under the PRGF. The remainder would become available upon completion of the fifth review. The definitions of the quantitative targets are provided in the attached Technical Memorandum of Understanding (TMU). To avoid the interruption of essential fiscal programs, in particular emergency measures to address the food crisis, the program continues to allow for the compensation of shortfalls in budget support from donors up to a limit of US\$50 million, through a program adjustor. The program allows full spending of any additional external support above and beyond the net amounts projected.

\section{Program Structural Measures}

25. Structural reforms will center on measures initiated earlier on in the program but still outstanding as well as newly-relevant measures, particularly in the areas of strengthening financial governance and reinforcing monetary policy independence (Table 3). To this end, we will seek prompt parliamentary approval of key laws tabled in mid-2007, including the banking law and a new customs code. We will begin implementing the new agreed recapitalization plan for the $\mathrm{BRH}$ : in particular, we will (i) increase interest payments by the MEF in line with the stipulations in the plan, (ii) establish a calendar for the replacement of the current unsecuritzed stock of central government debt to the BRH with the issuance of Treasury Bills (TB), with the first issuance of TB (in the amount of $\mathrm{G} 4.9 \mathrm{bn}$ ) to occur during FY 2010; (iii) establish, jointly by the MEF and BRH, the magnitude of government debt to the BRH to be treated under the recapitalization plan, and approve its consolidation by endSeptember 2009; and (iv) produce a memorandum of understanding between MEF and BRH on the financial terms of the TB to be issued, their tradability, and any other operational 
matters relevant for the implementation of the swap, consistent with the BRH recapitalization plan. We will continue to limit spending under ministerial current accounts and plan to monitor execution of off-budget spending under the emergency law as described in paragraph 18. The debt management unit in the Ministry of Economy and Finance will be reinforced, in preparation for issuance of MEF bonds and to ensure continued debt sustainability after the HIPC completion point. We intend to submit the draft organic DGI law to Parliament and publish regular reporting on our monetary policy goals and request that these benchmarks be reset for end-March. Finally, the cessation of BRH non-essential activities has been implemented: the laws relating to the disengagement of the BRH from APN and SONAPI were approved by Parliament in late 2007 (originally structural benchmark for September 2009) and that relating to BRH disengagement from BPH was voted in November 2008. The approved laws will be published in the official journal "Le Moniteur" by end-March-2009. These measures are laid out in more detail in the attached Technical Memorandum of Understanding.

\section{Implementation of HIPC Triggers}

26. We are working to implement the outstanding conditions for reaching the HIPC floating completion point, in order to benefit from HIPC and MDRI debt stock reductions by mid-2009. We have agreed with the IMF and World Bank staffs on an indicative timeframe for completing the steps needed to reach the completion point, including completion of an annual progress report on the first year of PRSP implementation, data reconciliation for the HIPC DSA analysis, and a timeframe for implementing the outstanding HIPC triggers. The most challenging of these is parliamentary passage of a new procurement law, and six months' of auditing consistent with the new law. We will make every effort to ensure that the law is submitted to Parliament and passed very soon. 
Table 1. Haiti: Proposed Schedule of Disbursements

\begin{tabular}{lll}
\hline Amount & Date & Conditions for Disbursement 1/ \\
\hline SDR 28,100,000 & November 20,2006 & $\begin{array}{l}\text { Executive Board approval of the three-year arrangement } \\
\text { under the PRGF. Includes 25\% of quota in access } \\
\text { for repayment of EPCA purchases }\end{array}$ \\
SDR 7,600,000 & July 23, 2007 & $\begin{array}{l}\text { Observance of performance criteria for March } 2007 \text { and } \\
\text { completion of the first review under the PRGF arrangement. }\end{array}$ \\
SDR 7,600,000 & February 20,2008 & $\begin{array}{l}\text { Observance of performance criteria for September 2007 and } \\
\text { completion of the second review under the PRGF arrangement. }\end{array}$ \\
SDR 23,980,000 & June 23, 2008 & $\begin{array}{l}\text { Observance of performance criteria for March 2008 and } \\
\text { completion of the third review under the PRGF arrangement. }\end{array}$ \\
SDR 23,980,000 2/ & February 11, 2009 & $\begin{array}{l}\text { Observance of performance criteria for September 2008 and } \\
\text { completion of the fourth review under the PRGF arrangement. }\end{array}$ \\
SDR 15,790,000 2/ & June 29, 2009 & $\begin{array}{l}\text { Observance of performance criteria for March 2009 and } \\
\text { completion of the fifth review under the PRGF arrangement. } \\
\text { Observance of performance criteria for September 2009 and } \\
\text { completion of the sixth review under the PRGF arrangement. }\end{array}$ \\
\hline
\end{tabular}

1/ Other than the generally applicable conditions for the Poverty Reduction and Growth Facility (PRGF).

2/ At the time of the fourth review, a program augmentation of 30 percent of the quota (SDR 24.57 million) is proposed for the Board's consideration. This table incorporates the proposed phasing of the augmentation (20 percent of quota to be disbursed upon completion of the fourth review, and 10 percent of quota to be disbursed at the time of completion of the fifth review).

$3 /$ An extension of the program beyond its expiration date (November 19, 2009) could be considered if additional time is needed to complete the sixth review. 
Table 2. Haiti: Indicative Targets and Quantitative Performance Criteria, FY 2009

\begin{tabular}{|c|c|c|c|c|c|}
\hline & \multirow{3}{*}{$\begin{array}{r}\begin{array}{r}\text { Actual stock at } \\
\text { end- } \\
\text { Sep-08 }\end{array} \\
\text { - }\end{array}$} & \multicolumn{4}{|c|}{ Cumulative Flows since September 2008} \\
\hline & & Dec 08 & Mar 09 & Jun 09 & Sept. 09 \\
\hline & & Ind. Target & Test Date & Ind. Target & Test Date \\
\hline Net central bank credit to the NFPS (in millions of gourdes) & 20,541 & 1,915 & 3,500 & 3,400 & 349 \\
\hline Central Government & 20,607 & 2,100 & 3,500 & 3,400 & 349 \\
\hline Rest of NFPS $1 /$ & -67 & -185 & 0 & 0 & 0 \\
\hline Net domestic banking sector credit to the central government $2 /$ & 13,336 & 5,115 & 9,530 & 9,430 & 6,379 \\
\hline Domestic arrears accumulation of the central government $4 /$ & & 0 & 0 & 0 & 0 \\
\hline \multicolumn{6}{|l|}{$\begin{array}{l}\text { New contracting or guaranteeing by the central government or the BRH } \\
\text { of nonconcessional external debt (In millions of U.S. dollars) } 4 / 5 \text { / }\end{array}$} \\
\hline Up to and including one year & & 0 & 0 & 0 & 0 \\
\hline Over one-year maturity & & 0 & 0 & 0 & 0 \\
\hline Net international reserves of central bank (in millions of U.S. dollars) - floor & 287 & 0 & -40 & -40 & -50 \\
\hline Change in currency in circulation & 13,030 & 1,800 & 950 & 1,100 & 1,250 \\
\hline Net domestic banking sector credit to the rest of the of the non-financial public sector & -112 & -185 & 0 & 0 & 0 \\
\hline Government total revenue, excl. grants (in millions of gourdes) & -- & 7,209 & 15,477 & 24,387 & 32,781 \\
\hline Government total expenditure, excl. ext-fin investment (in millions of gourdes) & -- & 12,251 & 27,043 & 36,685 & 45,398 \\
\hline
\end{tabular}

Sources: Ministry of Finance, Central Bank of Haiti, and Fund staff estimates.

1/ It includes non-budgetary autonomous organizations, local governments and public entities. It will be measured as the change, from September 2008, in Créances nettes sur le Secteur Public (i.e, Net Credit to the Non Financial Public Sector) minus the change in Créances nettes sur état (i.e, Net Credit to the Central Government), according to Table $10 \mathrm{R}$ of the BRH.

2/ It includes a reduction of government deposits in commercial banks, that were originated in Petrocaribe-related disbursements during FY2008. As of end-FY08, the balance of these deposits amounted to US $\$ 150$ million. The program includes a zero ceiling on commercial banks' gross credit to the

central government, on a continuous basis. The disaccumulation of deposits mainly finances hurricane-related reconstruction spending. See TMU.

3 / For program monitoring purposes, NDA is defined as monetary base minus Program NIR in gourde terms. Program exchange rate of G41 per US\$.

4/ On a continuous basis.

5/ Excludes guarantees granted to the electricity sector in the form of credit/guarantee letters. 
Table 3. Structural Measures for FY 2009

\begin{tabular}{|c|c|}
\hline Performance criteria & \\
\hline $\begin{array}{l}\text { Provide, along with normal monthly budget execution tables, execution of emergency } \\
\text { spending, by normal budget classification. }\end{array}$ & $\begin{array}{c}\text { Quarterly during the } \\
\text { arrangement period, starting } \\
\text { on March 31, } 2009 \text { and until all } \\
\text { off-budget emergency } \\
\text { spending is executed }\end{array}$ \\
\hline Limit to 10 percent nonwage current spending through current accounts. & $\begin{array}{l}\text { Quarterly (evaluated at end- } \\
\text { March and end-September } \\
\text { 2009) }\end{array}$ \\
\hline Legislative passage of new banking law. & End-September 2009 \\
\hline Implement first stage of BRH recapitalization plan. & End-September 2009 \\
\hline \multicolumn{2}{|l|}{ Benchmarks } \\
\hline $\begin{array}{l}\text { Set up and train debt management unit in MEF - both to use centralized external debt } \\
\text { database, but also to manage domestic Treasury debt in context of BRH recapitalization. }\end{array}$ & End-September 2009 \\
\hline Legislative passage of customs code. & End-September 2009 \\
\hline $\begin{array}{l}\text { Publication of the laws relating to the disengagement of the BRH from APN, SONAPI and } \\
\text { BPH approved by Parliament in the official journal "Le Moniteur". }\end{array}$ & End-March 2009 \\
\hline Submit the organic DGI law to Parliament (reset from September 2008). & End-March 2009 \\
\hline $\begin{array}{l}\text { Initiate regular central bank reporting on monetary policy goals and implementation (reset } \\
\text { from September } 2008 \text {. }\end{array}$ & End-March 2009 \\
\hline
\end{tabular}




\section{AtTaChment III. Haiti-TeChniCAL Memorandum OF Understanding}

1. Haiti's performance under the program supported by the Poverty Reduction and Growth Facility (PRGF) will be assessed on the basis of the observance of quantitative performance criteria as well as compliance with structural performance criteria and benchmarks. This Technical Memorandum of Understanding (TMU) defines the quantitative and structural performance criteria and indicative targets for the period October 2008September 2009, specified in Tables 1 and 2 of the Memorandum on Economic and Financial Policies (MEFP). It also lays down the monitoring and reporting requirements. The quantitative performance criteria under the program are set for end-March 2009 and endSeptember 2009. Targets for end-December 2008 and end-June 2009 are indicative.

\section{DEFINITIONS}

\section{A. Net BRH Credit To The Central Government ${ }^{1}$}

2. The change in net BRH credit to the central government is defined as, and will be measured using:

a. Change in net domestic credit to the central government from the BRH according to Table 10R of the BRH for end-September 2008.

b. Change in the stock of project accounts ("Comptes de projets") included in Table 10R of the BRH will be excluded from change in net domestic credit to the central government as defined above. ${ }^{2}$

c. Change in the stock of Special Accounts ("Comptes Speciaux") and seized values ("Valeurs Saisies UCREF") included in Table 10R of the BRH will be excluded from the change in net domestic credit to the central government as defined above. ${ }^{3}$

\footnotetext{
${ }^{1}$ The central government comprises the presidency, prime minister's office, parliament, national courts, treasury, line ministries and "organismes déconcentrés". It includes expenditures financed directly by foreign donors through ministerial accounts (comptes-courants).

${ }^{2}$ Project accounts ("Comptes de projets") refer to government accounts at the BRH which can only be used with the authorization of donors (similar to escrow accounts), i.e., their use is beyond the control of the central government. If included, movements in these accounts would appear as BRH credit to the government. See Section E for the treatment of PetroCaribe-related resources.

${ }^{3}$ Special Accounts (“Comptes Speciaux") refer to US\$-denominated central government sight deposits at the BRH. The balance of these accounts increase with the proceeds of the sales of in-kind aid (in the form of wheat, maize, rice, etc.) received by the Haitian government; these proceeds are earmarked to finance specific projects and cannot be used by the Central Government without the explicit authorization of respective donors. Thus, resources under the direct control of the government do not form part of 2.b and/or 2.c as defined above.
} 
3. The changes will be measured on a cumulative basis from the stock at end-September 2008.

\section{B. Net Domestic Banking Sector Credit to Central Government}

4. The change in net domestic banking sector credit to the central government is defined as, and will be measured, using:

a. Change in the stock of net domestic credit to the central government from the $\mathrm{BRH}$ according to Table 10R of the BRH; plus, the change in the stock of net domestic credit to the central government from domestic banks according to Table 20R of the BRH.

b. The change in the stock of project accounts ("Comptes de projets"), as defined in $2 . \mathrm{b}$ above, will be excluded from the change in net domestic banking sector to the Central Government.

c. The change in the stock of Special Accounts ("Comptes Speciaux") and seized values ("Valeurs Saisies UCREF"), as defined in 2.c above, will be excluded from the change in net domestic banking sector to the Central Government.

In addition, the ceiling for the change in gross credit from domestic banks to the central government will be set at zero continuously throughout the program period.

5. The changes will be measured on a cumulative basis from the stock at endSeptember 2008.

\section{Net International Reserves}

6. The change in net international reserves will be measured using:

a. Change in net foreign assets ("Réserves de change nettes" of the BRH Table 10R for 2008 and 2009); ${ }^{4}$

b. Minus the change in foreign currency deposits of commercial banks at the BRH ("Dépôts à vue en US\$ et en EURO des BCM à la BRH" of the BRH Table 10R).

c. Minus the change in the stock of project accounts ("Comptes de projets") as defined in $2 . b$ above.

d. Minus the change in the stock of Special Accounts ("Comptes Speciaux") and seized values ("Valeurs Saisies UCREF") as defined in 2.c above.

\footnotetext{
${ }^{4}$ Letters of credit and guarantee ("Lettres de crédit" and "Lettres de garantie") are reported in Table 10R as part of BRH foreign liabilities ("Engagements exterieurs"), and therefore are already netted out of NIR.
} 
7. Data will be expressed in U.S. dollar terms and valued at the corresponding endperiod market exchange rate.

8. For definition purposes, net international reserves are the difference between the BRH's gross foreign assets (comprising gold, special drawing rights, all claims on nonresidents, and BRH claims in foreign currency on domestic financial institutions) and reserve liabilities (including liabilities to nonresidents of one-year maturity or less, use of Fund credit, and excluding trust funds). Swaps in foreign currency with domestic financial institutions and pledged or otherwise encumbered reserve assets are excluded from net international reserves.

9. The changes will be measured on a cumulative basis from the stock at endSeptember 2008.

\section{Net Domestic Assets of the BRH}

10. The change in net domestic assets of the BRH is defined as, and will be measured using:

a. Change in base money (program definition according to Section I. below);

b. Minus the change in the U.S. dollar amount of net international reserves (program definition according to section $\mathrm{C}$ above), converted into gourdes at the program exchange rate.

11. The program definition of net domestic assets of the BRH will use a program exchange rate of G 41 per U.S. dollar for the period October 2008-September 2009.

12. The changes will be measured on a cumulative basis from the stock at endSeptember 2008.

\section{E. PetroCaribe-Related Inflows in FY 2008 and FY 2009}

13. PetroCaribe-related inflows during FY 2008 amounted to US\$201 million. These inflows constitute direct external debt of the central government and will be spent during FY 2009, mainly in post-hurricane reconstruction projects, and hurricane-related relief. As of end-September 2008, US\$51.0 million of such inflows were held in US\$-denominated sight deposits of the central government at the BRH, and the remaining US\$149 million in US\$-denominated deposits of the central government at domestic commercial banks. These resources are under the direct control of the central government, for program purposes.

14. Any new PetroCaribe inflows occurring in FY 2009 will continue to be held in central government accounts in the banking system until new institutional arrangements are finalized. ${ }^{5}$ The nature of these resources will be determined by their originating contract and

\footnotetext{
${ }^{5}$ ALBA refers to "Alternativa Boliviarana de las Americas".
} 
the definition of debt in $\uparrow 15$ below. Debt will be public if the debtor is the public sector, as defined by GFS-2001 (Chapter 2-Section D).

\section{F. Nonconcessional External and Foreign-Currency Denominated Debt}

15. The definition of debt comprises all instruments, including new financial instruments that share the characteristics of debt, as set forth in paragraph No. 9 of the Guidelines on Performance Criteria with Respect to Foreign Debt (Decision No.12274-(00/85), August 24, 2000).

16. The concessional nature of debt will be determined on the basis of the commercial interest reference rates (CIRRs), as laid out by the Organization for Economic Cooperation and Development (OECD). A debt is defined as concessional if, on the date of signature, the ratio between the present value of the debt computed on the basis of reference interest rates and the face value of the debt is less than 65 percent (equivalent to a grant element of at least 35 percent).

17. Excluded from the ceiling are short-term import-related credits, rescheduling arrangements, borrowing from the Fund, and guarantees for the electricity sector in the form of letters of credit.

18. The ceilings for contracting and guaranteeing nonconcessional debt by the central government and the BRH will be set at zero continuously throughout the program period.

\section{G. Government Current Accounts}

19. Ministerial current accounts are mechanisms for channeling expenditures. In principle, the use of these accounts should be limited to unforeseen emergency outlays. The BRH will provide monthly information on the stock of these current accounts for the central government (as defined in footnote 1).

20. The target is calculated on a cumulative basis. The ceiling on the use of current accounts will be met if year-to-date (starting on October 1st) expenditure executed through current accounts is less than 10 percent of nonwage budget appropriations at the end-March and end-September test dates. 


\section{H. Arrears}

21. External payment arrears are defined as overdue payments (principal and interest) to non-residents on debt contracted and guaranteed by the central government, and will be defined according to the terms of indebtedness of each creditor. The criterion of zero accumulation of external arrears will be monitored on a continuous basis.

22. Domestic arrears are defined to include: (i) any bill that has been received by a spending ministry from a supplier for goods and services delivered (and verified) and for which payment has not been made within 45 days after the due date of payment; (ii) wage, salary, and other payment to government employees, including direct and indirect allowances, that were due to be paid in a given month but remained unpaid on the 30th of the following month; and (iii) interest or principal obligations which remain unpaid 30 days after the due date of payment. This definition excludes changes in the stock of arrears on account of interest, penalties and valuation changes.

\section{Base Money}

23. The change in base money is defined as, and will be measured using:

a. Change in the stock of currency in circulation from Table 10R of the $\mathrm{BRH}$.

b. Change in the stock of reserve deposits of commercial banks at the BRH, from Table 10R, using gourde sight deposits of commercial banks (Dépôts a vue en gourdes des BCM a la BRH) and cash-in-vault of commercial banks (Encaisses des BCM).

24. The changes will be measured on a cumulative basis from the stock at end-September 2008.

\section{QUARTERly AdJUSTMEnTS}

25. The quarterly performance criteria and indicative targets will be adjusted for the following amounts:

\section{A. Adjustment for Domestic Arrears Accumulation}

26. The ceilings for net BRH credit to the central government and the net domestic banking sector credit to the central government will be adjusted downwards for the amount of outstanding domestic arrears accumulation.

\section{B. Adjustment for PetroCaribe-Related Inflows in FY 2009}

27. The ceiling for net domestic banking sector credit to the central government will be adjusted downwards for any (new) PetroCaribe/ALBA-related inflows during FY 2009 into central government deposits in the domestic banking sector (which comprises domestic banks and the BRH), while the ceiling for net BRH credit to the central government will be 
adjusted downwards for the portion of such flows deposited at the BRH. Correspondingly, the floor for Net International Reserves of the BRH will be adjusted upwards for any portion of such new inflows that are deposited at government accounts at the BRH. ${ }^{6}$

28. In case all or/any portion of such new inflows were to constitute public debt (direct and/or contingent) of any form, such inflows will count as "Program Net External Financing" as defined and referred to in paragraphs 29 and 30. Any spending of any form (within or outside the budget) financed with such new inflows during FY 2009 will be considered as central government spending for program purposes. ${ }^{7}$ In this case, a similar adjustment to that described in paragraph 27 will apply: The ceiling for net domestic banking sector credit to the central government will be adjusted downwards for the difference between any (new) PetroCaribe/ALBA-related inflows during FY 09 and any spending financed with such flows. The ceiling for net BRH credit to the central government will be adjusted downwards (and the floor for Net International Reserves upwards), for the portion of unspent inflows deposited at the BRH.

\section{Adjustment for Net Program External Financing}

29. The program ceilings on BRH net credit to the central government, and on BRH net domestic assets and the floor on NIR reflect an assumed flow of net external financing, defined as disbursements of cash budgetary assistance, exceptional financing (including rescheduled principal and interest) and debt relief minus debt service.

30. If actual net external financing is lower than programmed net external financing, the ceilings on net BRH credit to the central government and on net BRH domestic assets will be adjusted upward, and the floor on NIR will be adjusted downward, by the amount of the difference between actual and programmed net external financing, converted into gourdes at the program exchange rate. The amount of this adjustment will be limited to US\$50 million. The adjuster will be calculated on a cumulative basis from October 1, 2008.

Program Net External Financing

(In millions of U.S. dollars)

\begin{tabular}{|lcccc|}
\hline & $\begin{array}{c}\text { December } \\
2008\end{array}$ & $\begin{array}{c}\text { March } \\
2009\end{array}$ & $\begin{array}{c}\text { June } \\
2009\end{array}$ & $\begin{array}{c}\text { September } \\
2009\end{array}$ \\
\hline Program net external financing & 20.3 & 40.3 & 37.6 & 88.8 \\
\hline
\end{tabular}

\footnotetext{
${ }^{6}$ In other words, the baseline program assumes these flows to be zero.

${ }^{7}$ Net program external financing in the form of inflows from PetroCaribe/ALBA during FY 09 are assumed to be zero.
} 


\section{Adjustment for Lower Utilization of FY 2008-Related PetroCaribe Inflows}

31. Under the program, all PetroCaribe-related inflows received in FY 2008 are projected

to be spent during FY 2009, mainly in reconstruction-related investment projects and hurricane-related relief. In case such inflows are not used completely during FY 2009, (and to ensure that these projects remain adequately financed), (i) the quarterly FY 2009 ceiling for net domestic banking sector credit to the central government will be adjusted downwards for any unspent amount deposited at the domestic banking system, while the ceiling for net $\mathrm{BRH}$ credit to the central government will adjusted downwards for any portion of such unspent resources deposited at the BRH; and (ii) for the respective amount deposited at the $\mathrm{BRH}$, the net international reserve floor will be adjusted upwards. The structural performance criterion to fully report emergency spending applies on a quarterly basis until full utilization of PetroCaribe-related inflows received in 2008.

\section{Clarification of Structural Performance Criteria}

\section{A. Public Financial Management}

32. The performance criterion to monitor the use and accounting of the emergency offbudget public spending involves the provision, at end-March 2009 and quarterly afterwards (until all off-budget emergency spending is executed), of tables reporting the emergency spending according to normal budget classification (alongside with the standard monthly execution tables). These tables will be posted in the Ministry of the Economy and Finance's (MEF) website as is currently the practice with TOFE tables.

33. The benchmark for the setting up of a Debt Management Unit (DMU) at the MEF involves the (i) the definition of the function to be performed by the DMU as well as its location within the MEF organizational chart, by means of a MEF internal regulation; (ii) the assignment of personnel to comply with its basic functions; (iii) the adoption of a debt management software that could be linked to SYSDEP, as well as the training of relevant (Back Office) DMU staff in its operation; (iv) the centralization of the recording and storage of all public debt data at the DMU; (v) the production of a draft operations manual; (vi) the drafting of memoranda of understanding between the MEF and other ministries and the BRH to clearly establish responsibilities and avoid overlappings.

34. Regarding the benchmark for the legislative passage of the customs code, we will inform staff in case substantial changes to the draft law are proposed by Parliament. Regarding the benchmark for submission to Parliament of the organic DGI law, the proposed law will reflect international best practices as reflected in comments by IMF experts, as relevant for Haiti.

\section{B. Monetary Policy and Financial Sector}

35. The performance criterion to implement the first stage of the BRH recapitalization plan involves: (i) increasing interest payments on the stock of central government debt to the BRH to G1.1 billion in FY 2009; (ii) establishing a calendar for the replacement of the current unsecuritzed stock of central government debt to the BRH with the issuance of Treasury Bills (TB): the first such issuance (in the amount of G4.9 bn) is scheduled to occur 
during FY 2010; (iii) establishing, jointly by the MEF and BRH, the magnitude of government debt to the BRH to be treated under the recapitalization plan, and approving its consolidation by end-September 2009; and (iv) producing a memorandum of understanding between MEF and BRH on the financial terms of the TB to be issued, their tradability, and any other operational matters relevant for the implementation of the swap, consistent with the $\mathrm{BRH}$ recapitalization plan.

36. The performance criterion for legislative passage of the new banking law involves, as agreed, the approval by Parliament of a law consistent with Basel Core Principles. The approved law shall provide the BRH with adequate powers and legal protection for regulating and supervising bank operations; and establish a framework that allows the BRH to impose reorganization and liquidation measures on banks in a timely and forceful way.

\section{Provision OF INFORMATION}

37. To ensure adequate monitoring of the program, the authorities will provide daily, weekly and monthly monetary and fiscal indicators to IMF staff, as well as other data upon request.

\section{A. Daily}

38. Monetary Indicators: (a) Exchange rate; (b) Volume of foreign exchange transactions, of which BRH sales and purchases; (c) Gross international reserves; and (d) Net international reserves.

39. These data will be reported with maximum two-day lag (14-day final).

\section{B. Weekly}

40. Monetary Indicators: (a) Stock of BRH bonds; (b) Deposits at commercial banks (in gourdes and U.S. dollars); (c) Credit to private sector (in gourdes and U.S. dollars); (d) Credit to central government and public sector (net); and (e) Currency in circulation.

41. Fiscal Indicators: (a) Revenues (internal, external, other) and (b) Expenditures on cash basis (wages and salaries, goods and services, external debt, current accounts).

42. These data will be reported with maximum five-day lag preliminary data (four weeks for final data).

\section{Monthly}

43. Table $10 \mathrm{R}$ and Table $20 \mathrm{R}$ with a maximum of 30-day lag final data.

44. Tableau on the comptes courants with a maximum of 30-day lag final data.

45. "Project Accounts", by donor, with a maximum of 30-day lag final data

46. Tableau de trésorerie de devises with a maximum of 30-day lag final data. 
47. Tableau des Operations Financières de l'Etat, including attached tables reporting the execution of off-budget emergency spending (within 14 days).

48. Table underlying the TOFE which enables the determination of checks in circulation and balance on investment project accounts.

49. Set of external debt tables with a maximum 30-day lag final data.

50. Report of revenue collection of DGI (Rapport d'activités), with a maximum 30-day lag final data.

51. Tables of revenue collection of AGD (Indicateurs d'activités aux ports, Rapport analytique des perceptions douanières à l'importation), with a maximum 30-day lag final data.

52. Balance of Bureau de Monetization accounts, including those movements related with flows linked to the ALBA-PetroCaribe agreement. Balance of PetroCaribe/ALBA-related deposits at commercial banks and/or the BRH, with a maximum 30-day lag final data, including those related with the Fonds d'Urgence at the BRH (both gourde-denominated and foreign currency-denominated).

\section{Quarterly}

53. Report on poverty-reducing expenditures, with a maximum 30-day lag final data.

\section{E. Other Information}

54. The authorities will share with staff the by-laws of the new binational (Venezuela-Haiti) entity (as soon as they are enacted), including any and all needed information to assess the nature of such new entity; the authorities will also share with staff the financing terms of any financing received by such entity, including any and all information needed to assess whether any financing flows received by such new entity constitute public debt (direct and/or contingent) of any form. 


\section{INTERNATIONAL MONETARY FUND}

\section{HAITI}

\section{Debt Sustainability Analysis Update}

Prepared by the Staffs of the International Monetary Fund and the International Development Association

Approved by Gilbert Terrier and Dominique Desruelle (IMF) and Carlos Braga and Rodrigo A. Chaves (IDA)

February 2, 2009

The debt sustainability analysis (DSA) was prepared jointly by Bank and Fund staffs in accordance with the standardized Debt Sustainability Framework (DSF) methodology for low-income countries (LICS). The DSA has also benefited from consultation with InterAmerican Development Bank staff. As in the previous DSA, ${ }^{1}$ the findings indicate the risk of external debt distress is high given a present value $(P V)$ of debt-to-exports ratio that is above the relevant policy-dependent threshold for an extended period in the baseline scenario and higher in alternative and shocks scenarios. ${ }^{2}$ Haiti's weak export base is a key factor in its high risk of debt distress. An alternative scenario reflecting full delivery of HIPC and MDRI relief in FY 2009 has been included and shows that, following relief, Haiti's debt would remain below its indicative thresholds throughout the projection period. It is anticipated that the next LIC DSA will be prepared at the time of HIPC completion point.

\section{BACKGROUND}

1. Haiti's public debt as of end-September 2008 is estimated at about 29 percent of GDP. Most of the debt is owed to external creditors (25 percent of GDP), mainly the InterAmerican Development Bank (42 percent of total external debt), the World Bank (34 percent), and bilateral creditors (15 percent). Most domestic public debt corresponds to Central Bank gourde-denominated obligations vis-a-vis the domestic banking system.

\footnotetext{
${ }^{1}$ Country Report No. 08/117

${ }^{2}$ Haiti is classified as a weak performer based on its three-year average score of 2.85 on the World Bank's Country Policy and Institutional Assessment index (CPIA). For a weak performer, the indicative thresholds for external debt sustainability are a PV of debt-to-GDP ratio of 30 percent, a PV of debt-to-exports ratio of 100 percent, a PV of debt-to-revenue ratio of 200 percent, a debt-service-to-exports ratio of 15 percent, and a debt service-to-revenue ratio of 25 percent. The DSF defines "weak" policy performers as those with CPIA ratings below 3.25 .
} 


\section{Upon reaching its HIPC completion point, Haiti will benefit from HIPC and} MDRI debt relief on an irrevocable basis. Haiti received a Paris Club treatment on Cologne terms in December 2006 and has received interim relief from multilateral creditors under the HIPC initiative. Haiti is working to fulfill remaining completion point triggers and hopes to reach its completion point under HIPC and also receive additional MDRI relief in FY 2009. An additional custom scenario has been included to show the effect of HIPC/MDRI relief on Haiti's debt ratios.

3. Haiti is taking steps to strengthen its debt management capacity. With the help of UNCTAD and the World Bank, Haiti is creating a single external debt database that will facilitate information sharing between the finance ministry and central bank. In addition, as part of the HIPC Capacity Building Program, Haiti is working with the Center for Latin American Monetary Studies (CEMLA) to enhance its debt management capacity.

\section{Assumptions}

\section{The medium-term assumptions for the DSA have been revised to reflect a} number of severe shocks. These include food and fuel price spikes early in 2008 that led to an augmentation of the PRGF arrangement by 20 percent of quota, four successive hurricanes and tropical storms in August and September that caused losses amounting to 15 percent of GDP, and the effect of the international financial crisis on remittances and exports, which is only partly offset by falling international food and oil prices. The main assumptions for the DSA are summarized below (Table A1 includes the medium-term macroeconomic framework):

- Growth and inflation: GDP is assumed to be lower than in the previous DSA in FY 2008 (now projected to be 1.3 percent) and FY 2009 (now projected to be 2.5 percent) due to the impact of the natural disasters and slower global growth, while in the medium-term the real rate of growth is assumed to converge to 4.5 percent, as in the previous DSA. Prices are projected to fall from recent highs such that the GDP deflator (17 percent in 2008) would average 8.1 percent for 2008-2017 and 5 percent for 2018-28.

- $\quad$ Fiscal policy: After an initial deterioration in the overall fiscal deficit including grants to 3.9 percent of GDP in 2009, reflecting large near-term spending needs, this measure would improve to average 2.4 percent of GDP for 2008-17 and 1.2 percent of GDP for 2018-28.

- $\quad$ Exports of goods and services: decreased significantly in percent of GDP in FY 2008 and are expected to fall again in FY 2009 to 10.6 percent of GDP, and recover afterwards, averaging 13.4 percent of GDP for the 2018-28 period. The decrease in FY 2008 is explained by retooling in the export assembly industry to respond to buyer 
demands, as well as the initial impact of the natural disasters during late FY 2008. Export projections for FY 2009 were lowered both due to the negative impact of the hurricanes on agricultural production and tourism receipts, and reduced export demand linked to the economic slowdown in Haiti's main export markets. This is in contrast with the previous DSA, which assumed that the HOPE initiative would bring about strong export growth during FY 2009-10; the current DSA also assumes a positive impact of the HOPE initiative, but only beginning in FY 2010, after reconstruction of basic infrastructure. ${ }^{3}$

- Imports of goods and services: After a sharp jump in 2008 to 40 percent of GDP due largely to high oil and food prices, imports of goods and services are projected at 38.5 percent of GDP in 2009 and would gradually decline, averaging 36.4 percent of GDP for the 2018-28 period.

- $\quad$ Remittances: Private transfers are now expected to decrease during FY 2009 to 16 percent of GDP due to the effects of the international financial crisis (the previous DSA assumed an increase) before recovering thereafter to average 17.7 percent for 2008-17 and 16.9 percent for 2018-28.

- $\quad$ As in the previous DSA and reflecting standard practice for countries in the interim period (between HIPC decision and completion points), the updated baseline scenario assumes interim HIPC relief but neither HIPC completion point nor MDRI relief. ${ }^{4}$

\section{The main differences with the baseline scenario in the previous DSA are as}

\section{follows:}

- $\quad$ Updated macroeconomic framework, as described above;

- Incorporation of US\$197 million (2.9 percent of GDP) of PetroCaribe-related debt, that will be mainly used to finance, during FY 2009, hurricane-related humanitarian and reconstruction spending; ${ }^{5}$

\footnotetext{
${ }^{3}$ HOPE refers to Haitian Hemispheric Opportunity through Partnership Encouragement Act; it provides for preferential access of Haitian apparel exports to the U.S. market.

${ }^{4}$ See Staff Guidance Note on the Application of the Joint Bank-Fund Debt Sustainability Framework for LowIncome Countries, available at www.imf.org and www.worldbank.org.

${ }^{5}$ These PetroCaribe-related resources constitute public external debt and are treated as such for the purpose of calculating their impact on gross public debt.
} 
- Incorporation of the June PRGF augmentation of 20 percent of quota (US\$26.6 million) and the current proposal for a PRGF augmentation of 30 percent of quota (US\$37 million), to be disbursed in two tranches (20 percent upon completion of the fourth PRGF review and 10 percent upon completion of the fifth PRGF review).

6. The baseline scenario does not include future PetroCaribe-related public debt creating flows, as it is unclear: (i) what their magnitudes will be; (ii) when they will be disbursed; (iii) the form that they will take (i.e., whether they will be public or private external debt); ${ }^{6}$ and (iv) given the steep drop in oil prices, whether they will continue to materialize.

\section{External DebT Sustainability}

\section{Haiti's external debt relative to exports remains high in the baseline scenario} (see Figure A1 and Tables A2 and A3 for the evolution of external debt ratios under the baseline and alternative/shock scenarios). The updated macroeconomic framework and the higher loan disbursements in FY 2009 worsen the expected path of Haiti's debt ratios compared with the previous DSA. In particular, the PV of external debt-to-exports ratio will increase as a consequence of higher nominal debt and lower exports, reaching 159 percent of GDP in 2013. The ratio declines subsequently as the impact of the HOPE initiative on export growth kicks in, but remains above the 100 percent of GDP threshold throughout the projection period, reflecting Haiti's weak export base. ${ }^{7}$ The projected increase in the PV of the debt-to-exports ratio reflects both an increase in the NPV of external debt-to-GDP and a decrease in the ratio of exports of goods and services-to-GDP. In the baseline scenario, other debt stock and debt service ratios remain well below the relevant thresholds throughout the projection period.

\section{On account of the high initial debt ratios, key thresholds are breached when} sensitivity analyses are conducted. The analysis shows Haiti to be particularly vulnerable to lower non-debt creating flows such as remittances given a weak export base and to a combined shock to growth, exports, prices, and non-debt creating flows. Considering the most extreme shock for each indicator, the PV of debt-to-exports ratio would rise even farther above the threshold (to 214 percent in 2012 in the event of lower non-debt creating flows), the PV of debt-to-revenue ratio would breach the threshold (reaching 229 percent in 2011 with combined shocks), and the PV of debt-to-GDP ratio would remain only somewhat

\footnotetext{
${ }^{6}$ Under the most recent proposal, a Haitian-Venezuelan binational corporation would be created to intermediate the PetroCaribe resources and assume the liabilities.

${ }^{7}$ Also, remittances provide a reliable supply of foreign exchange. See previous LIC DSA in IMF Country Report 08/117.
} 
below the threshold ( 27 percent by 2012 with combined shocks). All external debt stock indicators then decline over the projection period but remain above the baseline. Debt service indicators rise somewhat in alternative and shock scenarios but the impact is relatively limited. The alternative scenario based on historical values for key variables shows lower debt ratios primarily due to a smaller current account deficit than in the baseline scenario, which in turn reflects low levels of external financing in the past during periods of social and political conflict.

\section{Public Debt Sustainability}

9. In the baseline scenario, public debt indicators increase initially and then decline somewhat during the projection period (see Figure A2 and Tables A4 and A5 for the evolution of public debt ratios under the baseline and alternative/shock scenarios). The PV of debt-to-GDP ratio rises to 20 percent in 2012 before falling to 16 percent by 2028 . The PV of debt-to-revenue ratio has a similar profile, reaching 134 percent in 2011 before falling to 93 percent by 2028. Debt service-to-revenue rises to 9 percent in 2015 before falling to 8 percent by 2028 .

\section{Alternative and shock scenarios put public debt on a steadily rising path} throughout the projection period. Instead of falling as under the baseline scenario, if growth is one standard deviation lower in 2008 and 2009, the PV of public debt-to-GDP ratio would grow to 34 percent in 2028 from 19 percent in 2008, while the PV of public debt-torevenue ratio would reach 192 percent in 2028 compared to 134 percent. Debt service-torevenue would rise but then remain flat under a growth shock. Keeping the primary balance at the 2008 level would lead to all public debt indicators rising consistently over the projection period. Using historical scenarios again yield lower debt levels.

\section{HIPC/MDRI DEBT RELIEF IN FY 2009}

\section{Debt relief at the HIPC completion point would substantially improve Haiti's} debt situation. A custom scenario has been added and is shown in Figures A1 and A2. Assuming that HIPC completion point and associated MDRI relief materialize in FY 2009, the PV of the external debt-to-exports ratio would fall below Haiti's indicative debt burden threshold of 100 percent in the medium-term immediately, and would remain just below the threshold throughout the projection period. Other indicators, which were already more favorable than the PV of debt-to-exports measure, would all be noticeably lower due to HIPC/MDRI relief.

\section{Conclusions}

12. Haiti's risk of external debt distress remains high given a PV of debt-to-exports ratio far above the indicative threshold in the baseline scenario. Alternative and shock scenarios highlight additional risks in terms of Haiti's debt stock measures, even while debt 
service indicators remain below the relevant thresholds in all scenarios. In terms of public debt, there is a risk of steadily rising debt ratios under alternative assumptions or in the event of shocks.

\section{Debt relief will help improve sustainability although additional measures are} needed. HIPC/MDRI relief would bring the PV of debt-to-exports ratio below the relevant indicative threshold, but with little cushion in the likely event of future shocks. Securing lasting debt sustainability will depend on: a prudent borrowing strategy, for which efforts to strengthen debt management should help, and steps to enhance Haiti's small export base, including through improved security and infrastructure to boost trade, especially given preferential opportunities. Better security and infrastructure could also catalyze higher foreign direct investment flows and reduce risks related to reliance on very high levels of remittances. Finally, sustained reform progress to bolster institutions and policy implementation capacity would increase Haiti's ability to handle a higher level of debt. 
Table A1. Haiti: Long-Term Macroeconomic Assumptions, 2008-28 Fiscal Year Ending September 30

\begin{tabular}{|c|c|c|c|c|c|c|c|c|c|c|}
\hline & \multirow[b]{2}{*}{2008} & \multirow[b]{2}{*}{2009} & \multirow[b]{2}{*}{2010} & \multirow[b]{2}{*}{2011} & \multirow[b]{2}{*}{2012} & \multirow[b]{2}{*}{2013} & \multirow[b]{2}{*}{2018} & \multirow[b]{2}{*}{2028} & \multicolumn{2}{|c|}{ Averages } \\
\hline & & & & & & & & & 2008-17 & 2018-28 \\
\hline \multicolumn{11}{|c|}{ (Annual percentage change) } \\
\hline \multicolumn{11}{|l|}{ National income and prices } \\
\hline GDP at constant prices & 1.3 & 2.5 & 2.7 & 3.2 & 3.3 & 3.4 & 4.3 & 4.5 & 3.2 & 4.5 \\
\hline GDP deflator & 17.0 & 12.0 & 10.0 & 8.0 & 7.0 & 6.0 & 5.0 & 5.0 & 8.1 & 5.0 \\
\hline Real GDP per capita (local currency) & -0.4 & 0.8 & 1.0 & 1.6 & 1.7 & 1.8 & 2.8 & 3.2 & 1.6 & 3.1 \\
\hline Consumer prices (period average) & 14.4 & 12.8 & 9.0 & 7.5 & 6.0 & 5.5 & 5.0 & 5.0 & 7.6 & 5.0 \\
\hline \multicolumn{11}{|l|}{ External sector (value in US\$) } \\
\hline Exports of goods and non-factor services & 7.3 & -4.3 & 12.7 & 6.3 & 6.5 & 5.9 & 7.5 & 7.5 & 6.3 & 7.6 \\
\hline Imports of goods and non-factor services & 22.4 & 2.8 & 2.7 & 6.7 & 7.2 & 3.9 & 5.5 & 5.7 & 6.5 & 5.7 \\
\hline \multicolumn{11}{|l|}{ Central government (value in G) } \\
\hline Total revenue and grants & 9.0 & 29.1 & 4.6 & 12.0 & 11.9 & 12.6 & 10.4 & 10.1 & 12.2 & 10.5 \\
\hline Central government revenue $1 /$ & 15.7 & 22.1 & 19.0 & 13.0 & 13.0 & 14.0 & 11.2 & 11.2 & 14.3 & 11.3 \\
\hline \multirow[t]{2}{*}{ Central government primary expenditure } & 37.2 & 34.9 & 5.3 & 8.9 & 8.5 & 8.8 & 10.2 & 10.3 & 14.1 & 10.5 \\
\hline & \multicolumn{8}{|c|}{ (In percent of GDP, unless otherwise indicated) } & & \\
\hline \multicolumn{11}{|l|}{ National income } \\
\hline Consumption & 102.2 & 96.9 & 97.2 & 96.3 & 95.9 & 94.0 & 94.0 & 89.8 & 95.9 & 91.9 \\
\hline Private & 95.6 & 89.8 & 90.2 & 89.5 & 89.2 & 87.4 & 87.1 & 82.4 & 89.2 & 84.6 \\
\hline Public & 6.6 & 7.1 & 6.9 & 6.8 & 6.6 & 6.5 & 7.0 & 7.5 & 6.7 & 7.3 \\
\hline Investment & 26.0 & 31.0 & 28.7 & 29.9 & 30.9 & 32.3 & 30.4 & 31.6 & 30.3 & 31.0 \\
\hline Private & 19.1 & 22.0 & 21.0 & 22.5 & 23.5 & 25.0 & 23.0 & 23.0 & 22.8 & 23.0 \\
\hline Public & 6.9 & 9.0 & 7.7 & 7.4 & 7.4 & 7.3 & 7.4 & 8.6 & 7.5 & 8.0 \\
\hline GDP per capita (US dollars) & 728 & 765 & 802 & 832 & 864 & 892 & 1,083 & 1,700 & 881 & 1,368 \\
\hline \multicolumn{11}{|l|}{ External sector } \\
\hline Non-interest current account deficit & -2.9 & -4.4 & -3.0 & -3.4 & -3.4 & -3.0 & -2.4 & -1.7 & -3.0 & -2.0 \\
\hline Exports of goods and non-factor services & 11.8 & 10.6 & 11.2 & 11.3 & 11.4 & 11.5 & 12.4 & 14.4 & 11.5 & 13.4 \\
\hline Imports of goods and non-factor services & 40.0 & 38.5 & 37.1 & 37.5 & 38.1 & 37.8 & 36.9 & 35.9 & 37.8 & 36.4 \\
\hline External current account balance 1/ & -8.6 & -11.9 & -8.4 & -8.2 & -8.5 & -8.1 & -7.0 & -4.9 & -8.3 & -5.9 \\
\hline External current account balance $2 /$ & -2.6 & -4.4 & -3.0 & -3.0 & -2.9 & -2.6 & -2.3 & -1.5 & -2.8 & -1.9 \\
\hline Liquid gross reserves (in months of imports of G\&S) & 2.9 & 2.6 & 2.7 & 2.9 & 3.0 & 3.2 & 3.1 & 2.9 & 3.0 & 3.0 \\
\hline \multicolumn{11}{|l|}{ Central government } \\
\hline Central government overall balance $2 /$ & -2.8 & -3.9 & -3.6 & -3.1 & -2.6 & -2.1 & -1.3 & -1.2 & -2.4 & -1.2 \\
\hline Total revenue and grants & 13.9 & 15.6 & 14.5 & 14.6 & 14.7 & 15.1 & 16.0 & 17.2 & 15.1 & 16.6 \\
\hline Central government revenue 1/ & 9.9 & 10.5 & 11.1 & 11.2 & 11.5 & 11.9 & 13.2 & 15.2 & 11.7 & 14.2 \\
\hline Central government primary expenditure & 16.1 & 18.9 & 17.6 & 17.2 & 16.9 & 16.7 & 16.9 & 18.1 & 17.0 & 17.5 \\
\hline
\end{tabular}

1/ Excluding grants

2/ Including grants 
Figure A1. Haiti: Indicators of Public and Publicly Guaranteed External Debt under Alternatives Scenarios, 2008-2028 1/
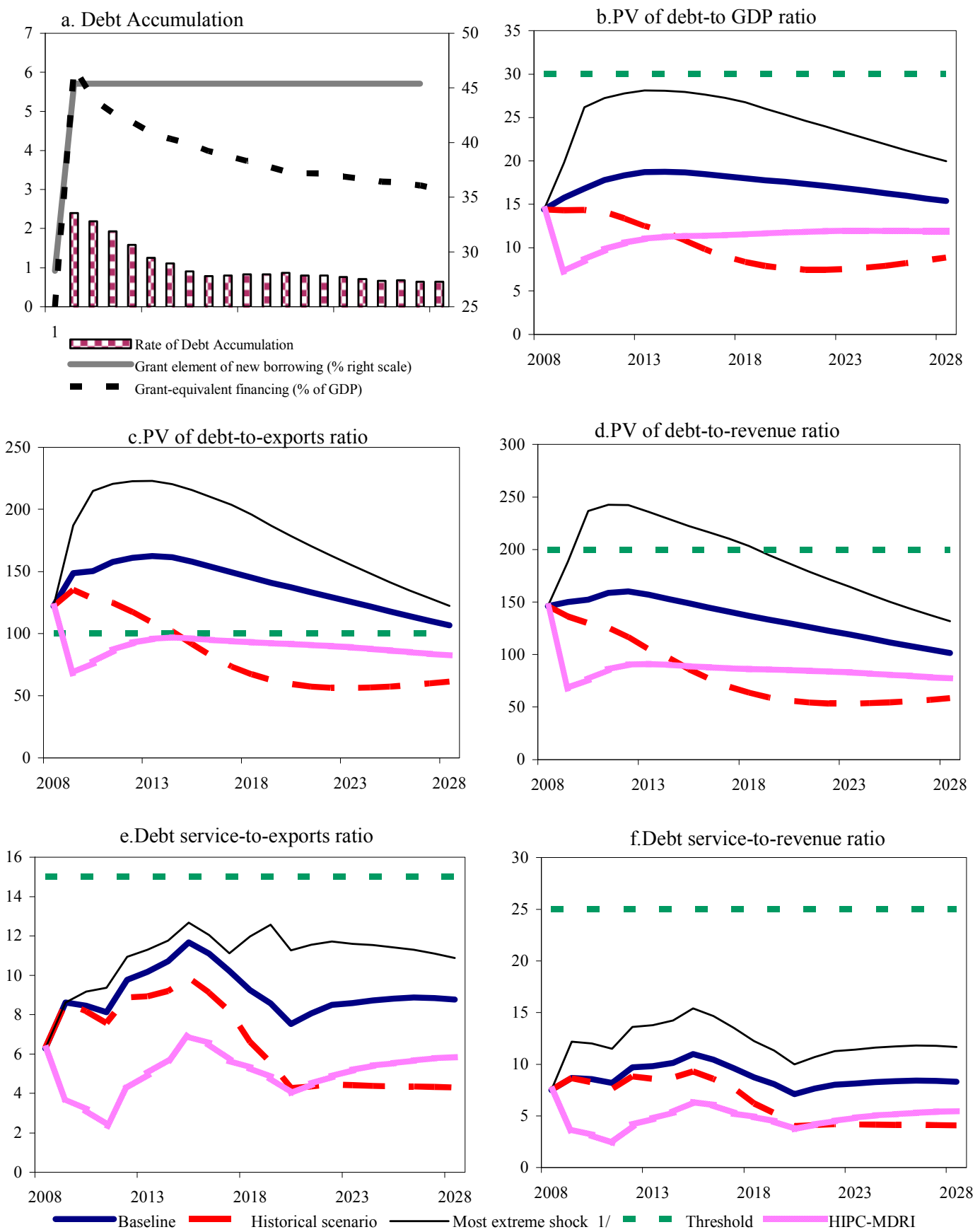

Source: Staff projections and simulations.

$1 /$ The most extreme stress test is the test that yields the highest ratio in 2018. In figure $b$. it corresponds to a Combination shock; in c. to a Non-debt flows shock; in d. to a Combination shock; in e. to a Non-debt flows shock and in picture f. to a One-time depreciation shock 
Table A2. Haiti: External Debt Sustainability Framework, Baseline Scenario, 2005-2028 1/ (In percent of GDP, unless otherwise indicated)

\begin{tabular}{|c|c|c|c|c|c|c|c|c|c|c|c|c|c|c|c|}
\hline & & ctual & & Historical 0 & Standard & & & Projecti & & & & & & & \\
\hline & 2005 & 2006 & 2007 & Average $0 \quad \mathrm{l}$ & tion & 2008 & 2009 & 2010 & 2011 & 2012 & 2013 & $\begin{array}{c}2008-2013 \\
\text { Average }\end{array}$ & 2018 & 2028 & $\begin{array}{c}2014-2028 \\
\text { Average }\end{array}$ \\
\hline External debt (nominal) 1/ & 34.3 & 28.1 & 24.4 & & & 25.1 & 26.6 & 28.6 & 30.0 & 30.7 & 30.9 & & 29.3 & 24.5 & \\
\hline $\mathrm{o} / \mathrm{w}$ public and publicly guaranteed $(\mathrm{PPG})$ & 34.3 & 28.1 & 24.4 & & & 25.1 & 25.7 & 27.7 & 29.2 & 30.0 & 30.2 & & 28.9 & 24.4 & \\
\hline Change in external debt & -0.3 & -6.2 & -3.6 & & & 0.6 & 1.5 & 2.0 & 1.5 & 0.7 & 0.2 & & -0.5 & -0.4 & \\
\hline $\begin{array}{l}\text { Identified net debt-creating flows } \\
\text { Non-interest current acount deficit }\end{array}$ & $\begin{array}{c}-9.4 \\
-1.8\end{array}$ & -5.6 & -6.9 & 10 & 12 & 1.9 & 3.6 & 1.5 & 1.4 & 1.5 & 1.2 & & 0.2 & $\begin{array}{c}-1.0 \\
1.3\end{array}$ & 17 \\
\hline $\begin{array}{l}\text { Non-interest current account deficit } \\
\text { Deficit in balance of goods and services }\end{array}$ & $\begin{array}{l}-1.8 \\
28.9\end{array}$ & 29.7 & 25.1 & & & 28.2 & 27.9 & 25.9 & 26.2 & 26.7 & $\begin{array}{r}2.3 \\
26.3\end{array}$ & & 24.5 & 21.5 & \\
\hline Exports & 14.0 & 14.4 & 12.7 & & & 11.8 & 10.6 & 11.2 & 11.3 & 11.4 & 11.5 & & 12.4 & 14.4 & \\
\hline Imports & 42.9 & 44.2 & 37.8 & & & 40.0 & 38.5 & 37.1 & 37.5 & 38.1 & 37.8 & & 36.9 & 35.9 & \\
\hline Net current transfers (negative $=$ inflow) & -30.5 & -28.3 & -24.7 & -23.8 & 5.7 & -25.3 & -23.5 & -22.9 & -22.9 & -23.4 & -23.3 & & -22.1 & -19.8 & -21.4 \\
\hline $\mathrm{o} / \mathrm{w}$ official & -7.6 & -7.9 & -6.4 & & & -6.0 & -7.5 & -5.4 & -5.2 & -5.5 & -5.5 & & -4.7 & -3.4 & \\
\hline Other current account flows (negative $=$ net inflow) & -0.2 & -0.1 & -0.2 & & & -0.6 & -0.2 & -0.3 & -0.7 & -0.8 & -0.7 & & -0.4 & -0.4 & \\
\hline Net FDI (negative = inflow) & -0.6 & -3.3 & -1.2 & -0.7 & 1.0 & -0.4 & -0.3 & -0.8 & -0.7 & -0.5 & -0.5 & & -0.9 & -1.5 & -1.1 \\
\hline Endogenous debt dynamics 2/ & -7.0 & -3.7 & -5.8 & & & 0.0 & -0.3 & -0.4 & -0.5 & -0.6 & -0.7 & & -0.9 & -0.8 & \\
\hline Contribution from nominal interest rate & -0.8 & 0.0 & 0.1 & & & 0.3 & 0.3 & 0.3 & 0.3 & 0.3 & 0.3 & & 0.3 & 0.3 & \\
\hline Contribution from real GDP growth & -0.5 & -0.7 & -0.8 & & & -0.3 & -0.6 & -0.7 & -0.9 & -0.9 & -1.0 & & -1.2 & -1.1 & \\
\hline $\begin{array}{l}\text { Contribution from price and exchange rate changes } \\
\text { Residual } 3-4,3 /\end{array}$ & -5.7 & -3.0 & -5.2 & & & & & & & & & & & & \\
\hline $\begin{array}{l}\text { Residual (3-4) 3/ } \\
\text { o/w exceptional financing }\end{array}$ & $\begin{array}{l}9.1 \\
1.0\end{array}$ & $\begin{array}{l}-\mathbf{0 . 6} \\
-0.2\end{array}$ & $\begin{array}{r}3.3 \\
-0.6\end{array}$ & & & $\begin{array}{l}-1.3 \\
-0.6\end{array}$ & $\begin{array}{l}-2.0 \\
-0.6\end{array}$ & $\begin{array}{l}\mathbf{0 . 4} \\
0.0\end{array}$ & $\begin{array}{l}\mathbf{0 . 1} \\
0.0\end{array}$ & $\begin{array}{r}-0.9 \\
0.0\end{array} \quad-100$ & $\begin{array}{r}-\mathbf{1 . 0} \\
0.0\end{array}$ & & $\begin{array}{rl}-0.7 & \\
0.0 & 0\end{array}$ & $\begin{array}{l}\mathbf{0 . 6} \\
0.0\end{array}$ & \\
\hline PV of external debt $4 /$ & $\ldots$ & $\ldots$ & 16.7 & & & 14.4 & 16.7 & 17.7 & 18.6 & 19.1 & 19.4 & & 18.4 & 15.5 & \\
\hline In percent of exports & & $\ldots$ & 131.2 & & & 122.1 & 157.7 & 158.3 & 164.8 & 167.5 & 168.3 & & 148.2 & 107.2 & \\
\hline PV of PPG external debt & ... & .... & 16.7 & & & 14.4 & 15.7 & 16.8 & 17.8 & 18.4 & 18.7 & & 18.0 & 15.4 & \\
\hline In percent of exports & .... & $\ldots$ & 131.2 & & & 122.1 & 148.8 & 150.3 & 157.6 & 161.1 & 162.5 & & 145.1 & 106.7 & \\
\hline In percent of government revenues & $\ldots$ & $\ldots$ & 165.4 & & & 146.0 & 150.0 & 152.2 & 158.8 & 160.2 & 156.9 & & 136.9 & 101.5 & \\
\hline Debt service-to-exports ratio (in percent) & 5.7 & 5.3 & 6.8 & & & 6.3 & 8.6 & 8.5 & 8.1 & 9.8 & 10.2 & & 9.2 & 8.8 & \\
\hline PPG debt service-to-exports ratio (in percent) & 5.7 & 5.3 & 6.8 & & & 6.3 & 8.6 & 8.5 & 8.1 & 9.8 & 10.2 & & 9.2 & 8.8 & \\
\hline PPG debt service-to-revenue ratio (in percent) & 8.2 & 7.7 & 8.5 & & & 7.5 & 8.7 & 8.6 & 8.2 & 9.7 & 9.8 & & 8.7 & 8.3 & \\
\hline $\begin{array}{l}\text { Total gross financing need (Billions of U.S. dollars) } \\
\text { Non-interest current account deficit that stabilizes debt ratio }\end{array}$ & $\begin{array}{l}-0.1 \\
-1.5\end{array}$ & $\begin{array}{r}-0.1 \\
7.6\end{array}$ & $\begin{array}{l}0.0 \\
3.8\end{array}$ & & & $\begin{array}{l}0.2 \\
1.7\end{array}$ & $\begin{array}{l}0.4 \\
2.6\end{array}$ & 0.2 & $\begin{array}{l}0.2 \\
1.2\end{array}$ & $\begin{array}{l}0.3 \\
1.9\end{array}$ & $\begin{array}{l}0.3 \\
2.1\end{array}$ & & $\begin{array}{l}0.3 \\
2.5\end{array}$ & $\begin{array}{l}0.2 \\
1.6\end{array}$ & \\
\hline Key macroeconomic assumptions & & & & & & & & & & & & & & & \\
\hline Real GDP growth (in percent) & 1.8 & 2.3 & 3.4 & 0.9 & 2.1 & 1.3 & 2.5 & 2.7 & 3.2 & 3.3 & 3.4 & 2.7 & 4.3 & 4.5 & 4.0 \\
\hline GDP deflator in US dollar terms (change in percent) & 19.7 & 9.7 & 22.7 & 6.0 & 13.6 & 14.4 & 4.2 & 3.8 & 2.1 & 2.2 & 1.4 & 4.7 & 1.4 & 1.4 & 1.8 \\
\hline Effective interest rate (percent) $5 /$ & -2.8 & 0.1 & 0.5 & -0.3 & 1.2 & 1.3 & 1.1 & 1.2 & 1.2 & 1.2 & 1.2 & 1.2 & 1.2 & 1.1 & 1.2 \\
\hline Growth of exports of G\&S (US dollar terms, in percent) & 18.3 & 15.5 & 12.1 & 8.1 & 11.4 & 7.3 & -4.3 & 12.7 & 6.3 & 6.5 & 5.9 & 5.7 & 7.5 & 7.5 & 7.5 \\
\hline Growth of imports of G\&S (US dollar terms, in percent) & 19.6 & 15.4 & 8.7 & 11.1 & 9.7 & 22.4 & 2.8 & 2.7 & 6.7 & 7.2 & 3.9 & 7.6 & 5.5 & 5.7 & 5.5 \\
\hline Grant element of new public sector borrowing (in percent) & & & & &.. & & 28.3 & 45.4 & 45.4 & 45.4 & 45.4 & & 45.4 & 45.4 & 45.4 \\
\hline Government revenues (excluding grants, in percent of GDP) & 9.7 & 10.0 & 10.1 & & & 9.9 & 10.5 & 11.1 & 11.2 & 11.5 & 11.9 & & 13.2 & 15.2 & 13.8 \\
\hline Aid flows (in Billions of US dollars) 7/ & 0.1 & 0.2 & 0.3 & & & 0.3 & 0.4 & 0.5 & 0.5 & 0.5 & 0.5 & & 0.5 & 0.8 & \\
\hline $\begin{array}{l}\mathrm{o} / \mathrm{w} \text { Grants } \\
\mathrm{o} / \mathrm{w} \text { Concessional loans }\end{array}$ & 0.1 & 0.2 & 0.3 & & & 0.3 & 0.4 & 0.3 & 0.3 & 0.3 & 0.3 & & 0.4 & 0.5 & \\
\hline $\begin{array}{l}\text { o/w Concessional loans } \\
\text { Grant-equivalent financing (in percent of GDP) } 8 \text { / }\end{array}$ & 0.0 & 0.0 & 0.0 & & & $\begin{array}{c}0.0 \\
\ldots .\end{array}$ & $\begin{array}{l}0.0 \\
6.1\end{array}$ & $\begin{array}{l}0.2 \\
5.3\end{array}$ & $\begin{array}{l}0.2 \\
5.0\end{array}$ & $\begin{array}{l}0.2 \\
4.7\end{array}$ & 0.2 & & 0.2 & $\begin{array}{l}0.3 \\
30\end{array}$ & 3.5 \\
\hline Grant-equivalent financing (in percent of external financing) 8/ & ... & $\ldots$ & $\cdots$ & & & ... & 71.2 & 70.1 & 71.8 & 72.9 & 74.9 & & 77.8 & 72.6 & 76.0 \\
\hline Memore & & & & & & & & & & & & & & & \\
\hline Nominal GDP (Billions of US dollars) & 4.3 & 4.8 & 6.1 & & & 7.1 & 7.6 & 8.1 & 8.5 & 9.0 & 9.4 & & 12.4 & 22.2 & \\
\hline Nominal dollar GDP growth & 21.8 & 12.2 & 26.9 & & & 15.8 & 6.8 & 6.6 & 5.4 & 5.5 & 4.8 & 7.5 & 5.8 & 6.0 & 5.9 \\
\hline PV of PPG external debt (in Billions of US dollars) & & & 1.0 & & & 1.0 & 1.2 & 1.4 & 1.5 & 1.7 & 1.8 & 16 & 2.2 & 3.4 & 08 \\
\hline
\end{tabular}

Source: Staff simulations.

1/ Includes both public and private sector external debt.

2 Derived as $[\mathrm{r}-\mathrm{g}-\mathrm{r}(1+\mathrm{g})] /(1+\mathrm{g}+\mathrm{r}+\mathrm{gr})$ times previous period debt ratio, with $\mathrm{r}=$ nominal interest rate; $\mathrm{g}=$ real GDP growth rate, and $\mathrm{r}=$ growth rate of GDP deflator in U.S. dollar terms.

3/ Includes exceptional financing (i.e., changes in arrears and debt relief); changes in gross foreign assets; and valuation adjustments. For projections also includes contribution from price and exchange rate changes.

$5 /$ Current-year interest payments divided by previous period debtsilc.

Historical averages and standard deviations are generally derived over the past 10 years, subject to data availability.

8/ Grant-equivalent financing includes grants provided directly to the government and through new borrowing (difference between the face value and the PV of new debt). 
Table A3.Haiti: Sensitivity Analysis for Key Indicators of Public and Publicly Guaranteed External Debt, 2008-2028 (In percent)

Projections

\begin{tabular}{l}
\hline PV of debt-to GDP ratio \\
\hline Paseline
\end{tabular}

Baseline

14

2009

2010

201

2012

2013

2018

2028

A. Alternative Scenarios

A1. Key variables at their historical averages in 2008-2028 1/

A2. New public sector loans on less favorable terms in 2008-2028 2

A3. HIPC \& MDRI Delivered in 2009

B. Bound Tests

B1. Real GDP growth at historical average minus one standard deviation in 2009-2010

B2. Export value growth at historical average minus one standard deviation in 2009-2010 3 ,

B3. US dollar GDP deflator at historical average minus one standard deviation in 2009-2010

B4. Net non-debt creating flows at historical average minus one standard deviation in 2009-2010 4/

B5. Combination of B1-B4 using one-half standard deviation shocks

B6. One-time 30 percent nominal depreciation relative to the baseline in 2009 5/

PV of debt-to-exports ratio

Baseline

A. Alternative Scenarios

A1. Key variables at their historical averages in 2008-2028 1/

A2. New public sector loans on less favorable terms in 2008-2028

B. Bound Tests

B1. Real GDP growth at historical average minus one standard deviation in 2009-2010

B2. Export value growth at historical average minus one standard deviation in 2009-2010 3

B3. US dollar GDP deflator at historical average minus one standard deviation in 2009-2010

B4. Net non-debt creating flows at historical average minus one standard deviation in 2009-2010 4/

B5. Combination of B1-B4 using one-half standard deviation shocks

B6. One-time 30 percent nominal depreciation relative to the baseline in 20095 /

$\begin{array}{rrrrrrrr}122 & 149 & 150 & 158 & 161 & 162 & \mathbf{1 4 5} & 107 \\ & & & & & & & \\ 122 & 135 & 128 & 125 & 117 & 109 & \mathbf{6 7} & 61 \\ 122 & 147 & 153 & 160 & 164 & 166 & \mathbf{1 4 8} & 112 \\ & & & & & & & \\ 122 & 149 & 150 & 157 & 161 & 162 & \mathbf{1 4 5} & 107 \\ 122 & 147 & 182 & 190 & 194 & 195 & \mathbf{1 7 4} & 125 \\ 122 & 149 & 150 & 157 & 161 & 162 & \mathbf{1 4 5} & 107 \\ 122 & 187 & 215 & 221 & 223 & 223 & \mathbf{1 9 6} & 122 \\ 122 & 162 & 208 & 214 & 216 & 217 & \mathbf{1 9 1} & 123 \\ 122 & 149 & 150 & 157 & 161 & 162 & \mathbf{1 4 5} & 107\end{array}$

PV of debt-to-revenue ratio

Baseline

A. Alternative Scenarios

A1. Key variables at their historical averages in $2008-2028$ 1/

A2. New public sector loans on less favorable terms in 2008-2028

B. Bound Tests

B1. Real GDP growth at historical average minus one standard deviation in 2009-2010

B2. Export value growth at historical average minus one standard deviation in 2009-2010 3

B3. US dollar GDP deflator at historical average minus one standard deviation in 2009-2010

B4. Net non-debt creating flows at historical average minus one standard deviation in 2009-2010 4/

B5. Combination of B1-B4 using one-half standard deviation shocks

B6. One-time 30 percent nominal depreciation relative to the baseline in 2009 5/
150

152

146

146

136

14

146

146
146

146
146

146

146
18

19

$\begin{array}{rrrrrrrr}4 & 14 & 14 & 14 & 13 & 13 & \mathbf{8} & 9 \\ 4 & 16 & 17 & 18 & 19 & 19 & \mathbf{1 8} & 16 \\ 4 & 7 & 9 & 10 & 11 & 11 & \mathbf{1 2} & 12\end{array}$

$4 \quad 16$

$6 \quad 18$

$\begin{array}{llllll}18 & 19 & 20 & 20 & \mathbf{1 9} & 17 \\ 18 & 19 & 19 & 19 & \mathbf{1 9} & 16 \\ 21 & 23 & 23 & 24 & \mathbf{2 3} & 19 \\ 24 & 25 & 25 & 26 & \mathbf{2 4} & 18 \\ 26 & 27 & 28 & 28 & \mathbf{2 7} & 20 \\ 24 & 25 & 26 & 26 & \mathbf{2 5} & 22\end{array}$


Table A3.Haiti: Sensitivity Analysis for Key Indicators of Public and Publicly Guaranteed External Debt, 2008-2028 (continued) (In percent)

\section{Debt service-to-exports ratio}

Baseline

A. Alternative Scenarios

A1. Key variables at their historical averages in 2008-2028 1/

A2. New public sector loans on less favorable terms in 2008-2028 2

B. Bound Tests

B1. Real GDP growth at historical average minus one standard deviation in 2009-2010

B2. Export value growth at historical average minus one standard deviation in 2009-2010 3/

B3. US dollar GDP deflator at historical average minus one standard deviation in 2009-2010

B4. Net non-debt creating flows at historical average minus one standard deviation in 2009-2010 4/

B5. Combination of B1-B4 using one-half standard deviation shocks

B6. One-time 30 percent nominal depreciation relative to the baseline in $20095 /$

Debt service-to-revenue ratio

Baseline

\section{A. Alternative Scenarios}

A1. Key variables at their historical averages in 2008-2028 1/

A2. New public sector loans on less favorable terms in 2008-2028 2

B. Bound Tests

B1. Real GDP growth at historical average minus one standard deviation in 2009-2010 B2. Export value growth at historical average minus one standard deviation in 2009-2010 3/

B3. US dollar GDP deflator at historical average minus one standard deviation in 2009-2010

B4. Net non-debt creating flows at historical average minus one standard deviation in 2009-2010 4/

B5. Combination of B1-B4 using one-half standard deviation shocks

B6. One-time 30 percent nominal depreciation relative to the baseline in 2009 5/

Memorandum item:

Grant element assumed on residual financing (i.e., financing required above baseline) 6/

\section{9}

6

(1)

9
9

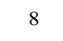

10

10

9

$\begin{array}{rrrrrr}8 & 8 & 9 & 9 & \mathbf{7} & 4 \\ 9 & 10 & 11 & 12 & \mathbf{1 1} & 7\end{array}$

$\begin{array}{ll}6 & 9 \\ 6 & 9 \\ 6 & 9 \\ 6 & 9 \\ 6 & 8 \\ 6 & 9\end{array}$

$\begin{array}{rr}8 & 8 \\ 10 & 10 \\ 8 & 8 \\ 9 & 9 \\ 9 & 9 \\ 8 & 8\end{array}$

$\begin{array}{rrrr}10 & 10 & \mathbf{9} & 9 \\ 11 & 12 & \mathbf{1 1} & 10 \\ 10 & 10 & \mathbf{9} & 9 \\ 11 & 11 & \mathbf{1 2} & 11 \\ 11 & 11 & \mathbf{1 1} & 11 \\ 10 & 10 & \mathbf{9} & 9\end{array}$

Source: Staff projections and simulations.

1/ Variables include real GDP growth, growth of GDP deflator (in US. dollar terms), non-interest current account in percent of GDP, and non-debt creating flows.

$2 /$ Assumes that the interest rate on new borrowing is by 2 percentage points higher than in the baseline., while grace and maturity periods are the same as in the baseline.

3/ Exports values are assumed to remain permanently at the lower level, but the current account as a share of GDP is assumed to return to its baseline level after the shock (implicitly assuming

an offsetting adjustment in import levels).

4/ Includes official and private transfers and FDI.

5/ Depreciation is defined as percentage decline in dollar/local currency rate, such that it never exceeds 100 percent.

6/ Applies to all stress scenarios except for A2 (less favorable financing) in which the terms on all new financing are as specified in footnote 2. 
Figure A2.Haiti: Indicators of Public Debt Under Alternative Scenarios, 2008-2028 1/

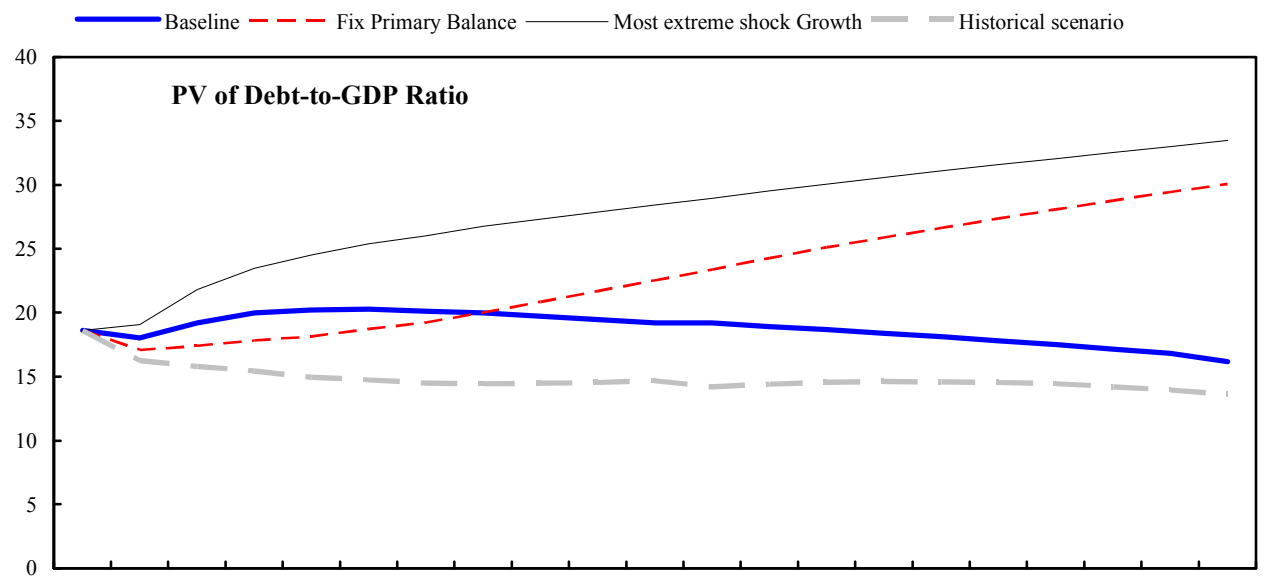

200820092010201120122013201420152016201720182019202020212022202320242025202620272028

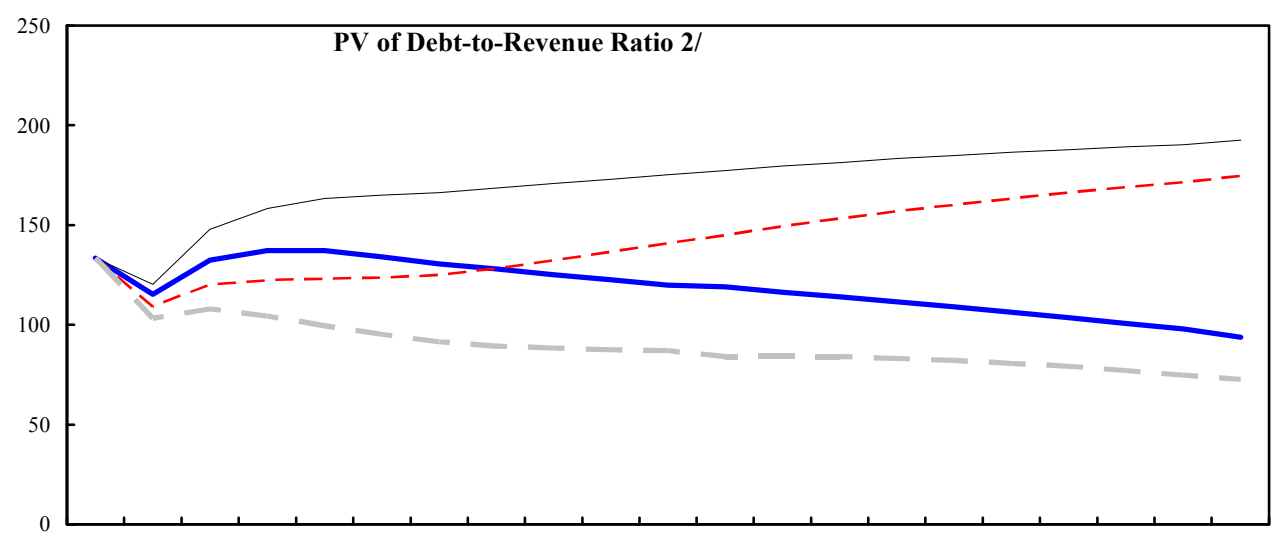

200820092010201120122013201420152016201720182019202020212022202320242025202620272028

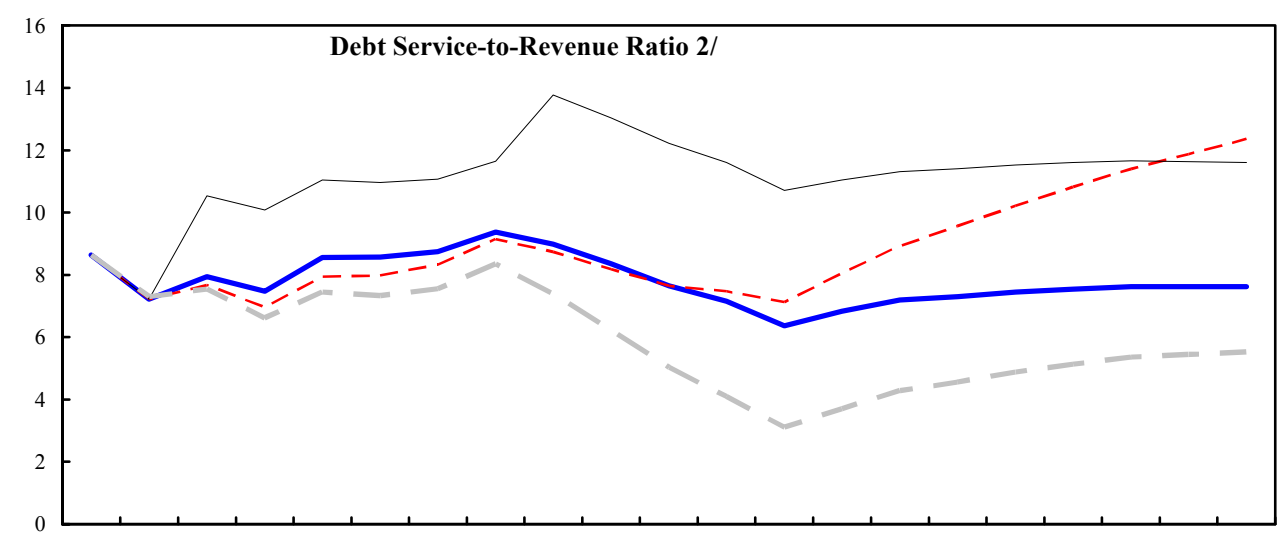

200820092010201120122013201420152016201720182019202020212022202320242025202620272028

Sources: Country authorities; and Fund staff estimates and projections.

$1 /$ The most extreme stress test is the test that yields the highest ratio in 2018.

$2 /$ Revenues are defined inclusive of grants. 
Table A4.Haiti: Public Sector Debt Sustainability Framework, Baseline Scenario, 2005-2028

(In percent of GDP, unless otherwise indicated)

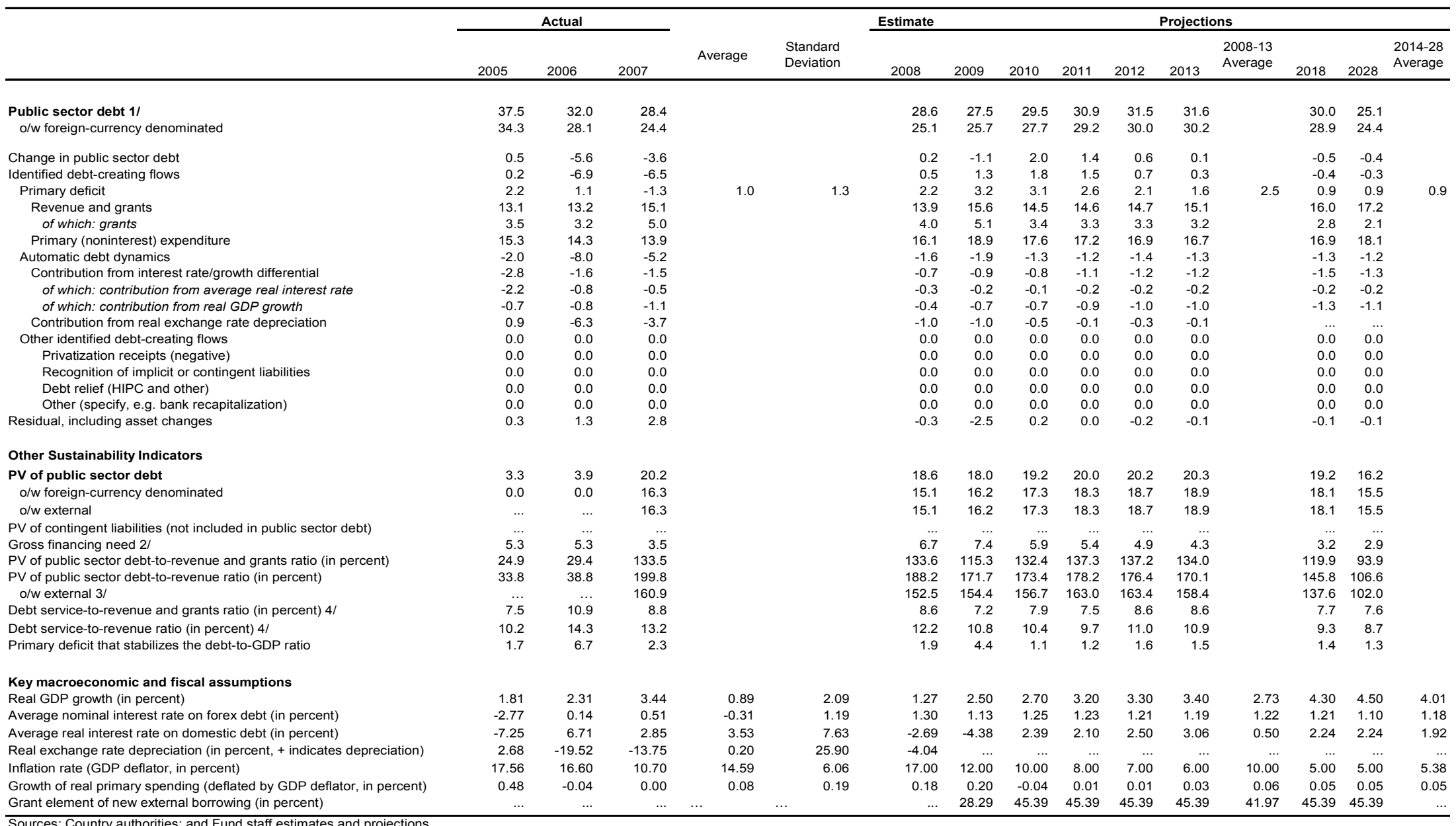

Sources: Country authorities; and Fund staff estimates and projections.

2/ Gross financing need is defined as the primary deficit plus debt service plus the stock of short-term debt at the end of the last period.

4/ Debt service is defined as the sum of interest and amortization of medium and long-term debt.

5/Historical averages and standard deviations and anerally derived over the past 10 years, subject to data availability. 
Table A5.Haiti: Sensitivity Analysis for Key Indicators of Public Debt 2008-2028

\begin{tabular}{|c|c|c|c|c|c|c|c|c|}
\hline & \multicolumn{8}{|c|}{ Projections } \\
\hline & 2008 & 2009 & 2010 & 2011 & 2012 & 2013 & 2018 & 2028 \\
\hline \multicolumn{9}{|l|}{ PV of Debt-to-GDP Ratio } \\
\hline Baseline & 19 & 18 & 19 & 20 & 20 & 20 & 19 & 16 \\
\hline \multicolumn{9}{|l|}{ A. Alternative scenarios } \\
\hline A1. Real GDP growth and primary balance are at historical averages & 19 & 16 & 16 & 15 & 15 & 15 & 15 & 14 \\
\hline A2. Primary balance is unchanged from 2008 & 19 & 17 & 17 & 18 & 18 & 19 & 23 & 30 \\
\hline A3. Permanently lower GDP growth $1 /$ & 19 & 18 & 19 & 21 & 21 & 21 & 23 & 29 \\
\hline \multicolumn{9}{|l|}{ B. Bound tests } \\
\hline B1. Real GDP growth is at historical average minus one standard deviations in $2009-2010$ & 19 & 19 & 22 & 23 & 24 & 25 & 28 & 33 \\
\hline B2. Primary balance is at historical average minus one standard deviations in $2009-2010$ & 19 & 17 & 18 & 18 & 19 & 19 & 18 & 15 \\
\hline B3. Combination of B1-B2 using one half standard deviation shocks & 19 & 17 & 17 & 19 & 20 & 20 & 22 & 26 \\
\hline B4. One-time 30 percent real depreciation in 2009 & 19 & 25 & 25 & 25 & 25 & 25 & 24 & 22 \\
\hline B5. 10 percent of GDP increase in other debt-creating flows in 2009 & 19 & 27 & 28 & 29 & 29 & 29 & 27 & 23 \\
\hline \multicolumn{9}{|c|}{ PV of Debt-to-Revenue Ratio 2/ } \\
\hline Baseline & 134 & 115 & 132 & 137 & 137 & 134 & 120 & 94 \\
\hline \multicolumn{9}{|l|}{ A. Alternative scenarios } \\
\hline A1. Real GDP growth and primary balance are at historical averages & 134 & 103 & 108 & 105 & 100 & 95 & 87 & 73 \\
\hline A2. Primary balance is unchanged from 2008 & 134 & 109 & 120 & 122 & 123 & 124 & 141 & 175 \\
\hline A3. Permanently lower GDP growth $1 /$ & 134 & 116 & 134 & 141 & 142 & 141 & 141 & 166 \\
\hline \multicolumn{9}{|l|}{ B. Bound tests } \\
\hline B1. Real GDP growth is at historical average minus one standard deviations in $2009-2010$ & 134 & 120 & 148 & 158 & 163 & 165 & 175 & 193 \\
\hline B2. Primary balance is at historical average minus one standard deviations in $2009-2010$ & 134 & 110 & 121 & 126 & 127 & 124 & 111 & 87 \\
\hline B3. Combination of B1-B2 using one half standard deviation shocks & 134 & 108 & 117 & 127 & 131 & 132 & 138 & 148 \\
\hline B4. One-time 30 percent real depreciation in 2009 & 134 & 157 & 171 & 172 & 169 & 163 & 148 & 128 \\
\hline B5. 10 percent of GDP increase in other debt-creating flows in 2009 & 134 & 173 & 193 & 197 & 196 & 190 & 170 & 133 \\
\hline \multicolumn{9}{|c|}{ Debt Service-to-Revenue Ratio $2 /$} \\
\hline Baseline & 9 & 7 & 8 & 7 & 9 & 9 & 8 & 8 \\
\hline \multicolumn{9}{|l|}{ A. Alternative scenarios } \\
\hline A1. Real GDP growth and primary balance are at historical averages & 9 & 7 & 8 & 7 & 7 & 7 & 5 & 6 \\
\hline A2. Primary balance is unchanged from 2008 & 9 & 7 & 8 & 7 & 8 & 8 & 8 & 12 \\
\hline A3. Permanently lower GDP growth $1 /$ & 9 & 7 & 8 & 8 & 9 & 9 & 9 & 12 \\
\hline \multicolumn{9}{|l|}{ B. Bound tests } \\
\hline B1. Real GDP growth is at historical average minus one standard deviations in $2009-2010$ & 9 & 7 & 9 & 8 & 10 & 10 & 10 & 14 \\
\hline B2. Primary balance is at historical average minus one standard deviations in $2009-2010$ & 9 & 7 & 8 & 7 & 8 & 8 & 7 & 7 \\
\hline B3. Combination of B1-B2 using one half standard deviation shocks & 9 & 7 & 8 & 7 & 8 & 8 & 8 & 11 \\
\hline B4. One-time 30 percent real depreciation in 2009 & 9 & 9 & 11 & 11 & 12 & 13 & 12 & 14 \\
\hline B5. 10 percent of GDP increase in other debt-creating flows in 2009 & 9 & 7 & 11 & 10 & 11 & 11 & 12 & 12 \\
\hline
\end{tabular}

Sources: Country authorities; and Fund staff estimates and projections.

1/ Assumes that real GDP growth is at baseline minus one standard deviation divided by the length of the projection period.

2/ Revenues are defined inclusive of grants. 
INTERNATIONAL MONETARY FUND

HAITI

Fourth Review Under the Three-Year Arrangement

Under the Poverty Reduction and Growth Facility, Request for Waiver of Performance Criterion and Augmentation of Access

\section{Informational Annex}

Prepared by the Western Hemisphere Department

(In consultation with other departments)

Approved by Gilbert Terrier and Dominique Desruelle

February 2, 2009

Contents

Page

Annexes

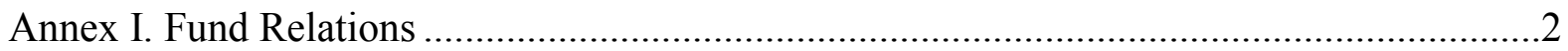

Annex II. Relations With The Inter-American Development Bank .....................................5

Annex III. Relations With The World Bank Group........................................................... 


\section{ANNEX I. Fund RELATIONS}

(As of September 30, 2008)

\section{Membership Status:}

Joined: September 08, 1953; Article VIII member

II. General Resources Account:

Quota

Fund holdings of currency

Reserve Position

Holdings Exchange Rate

III. SDR Department:

Net cumulative allocation

Holdings

IV. Outstanding Purchases and Loans:

PRGF Arrangements

$\begin{array}{rr}\text { SDR Million } & \text { \%Quota } \\ 81.90 & 100.00 \\ 81.83 & 99.92 \\ 0.07 & 0.08\end{array}$

SDR Million

\%Allocation

13.70

100.00

4.72

34.44

SDR Million

\%Quota

67.28

82.15

\section{Latest Financial Arrangements:}

\begin{tabular}{llcrr}
\multicolumn{1}{c}{ Type } & $\begin{array}{c}\text { Date of } \\
\text { Arrangement }\end{array}$ & $\begin{array}{c}\text { Expiration } \\
\text { Date }\end{array}$ & $\begin{array}{c}\text { Amount Approved } \\
\text { (SDR Million) }\end{array}$ & $\begin{array}{c}\text { Amount Drawn } \\
\text { (SDR Million) }\end{array}$ \\
PRGF & Nov 20, 2006 & Nov 19, 2009 & 90.09 & 67.28 \\
PRGF & Oct 18, 1996 & Oct 17, 1999 & 91.05 & 15.18 \\
Stand-By & Mar 08, 1995 & Mar 07, 1996 & 20.00 & 16.40
\end{tabular}

VI. Projected Payments to Fund 1/

(SDR Million; based on existing use of resources and present holdings of SDRs):

\begin{tabular}{lccccc} 
& \multicolumn{5}{c}{ Forthcoming } \\
\cline { 2 - 6 } & $\underline{2008}$ & $\underline{2009}$ & $\underline{2010}$ & $\underline{2011}$ & $\frac{2012}{5.62}$ \\
Principal & $\underline{0.23}$ & $\underline{0.57}$ & $\underline{0.57}$ & $\underline{0.57}$ & $\underline{0.56}$ \\
Total & $\underline{0.23}$ & $\underline{0.57}$ & $\underline{0.57}$ & $\underline{0.57}$ & $\underline{6.18}$
\end{tabular}

\footnotetext{
${ }^{1 /}$ When a member has overdue financial obligations outstanding for more than three months, the amount of such arrears will be shown in this section.
} 
VII. Implementation of HIPC Initiative:

Enhanced

I. Commitment of HIPC assistance

Framework

Decision point date

Nov 2006

Assistance committed

by all creditors (US\$ Million) ${ }^{1 /}$

Of which: IMF assistance (US\$ million)

(SDR equivalent in millions)

Completion point date

Floating

II. Disbursement of IMF assistance (SDR Million)

Assistance disbursed to the member

0.15

Interim assistance

0.15

Completion point balance

Additional disbursement of interest income ${ }^{2 /}$

Total disbursements

0.15

${ }^{1 /}$ Assistance committed under the original framework is expressed in net present value (NPV) terms at the completion point, and assistance committed under the enhanced framework is expressed in NPV terms at the decision point. Hence, these two amounts cannot be added.

${ }^{2 /}$ Under the enhanced framework, an additional disbursement is made at the completion point corresponding to interest income earned on the amount committed at the decision point but not disbursed during the interim period.

VIII. Implementation of Multilateral Debt Relief Initiative (MDRI): Not Applicable

\section{Exchange Arrangement}

Managed floating with no predetermined path for the exchange rate. The change from a fixed to managed floating regime took place in January 1990. Haiti's exchange system is free of restrictions on the making of payments and transfers for current international transactions. Since September 1991, all transactions have taken place at the free (interbank) market rate.

\section{Safeguards Assessment}

The update assessment of the Banque de la République d'Haiti (BRH) was concluded in September 2008. The authorities have made progress in implementing safeguards recommendations, but improvements are still needed in a number of areas. The 2007 audit has been recently completed and is expected to be published in the coming weeks. The 
qualitative analysis of the main differences between currently used accounting principles and IFRS did not reveal major differences and suggests that a gradual adoption of IFRS by the $\mathrm{BRH}$ is feasible. Another significant step was the adoption of the Audit Committee Charter in March 2007, followed by its constitution in February 2008. However, the capacity of this Committee needs to be strengthened. Vulnerabilities remain in the areas of foreign reserves management, the timely conduct of external audits, and timely production of audited financial statements. Since the update assessment in the context of the first augmentation was recently concluded, the conclusions of that assessment continue to be valid for the second augmentation of access.

\section{Article IV Consultation}

The last Article IV consultation was concluded by the Executive Board on July 9, 2007. Haiti is on a 24-month cycle.

\section{Technical Assistance}

Haiti has benefited from the following IMF technical assistance missions since 2005:

\begin{tabular}{|c|c|c|}
\hline Department & Dates & Purpose \\
\hline \multirow[t]{2}{*}{ FAD } & April 2005 & Public expenditure management \\
\hline & May 2005 & Tax policy and revenue administration \\
\hline \multirow[t]{5}{*}{$\mathrm{MCM}$} & March 2005 & Monetary operations \\
\hline & November 2005 & Implementation of a bond auction mechanism \\
\hline & May 2006 & Accounting of the central bank \\
\hline & March 2007 & Banking law (jointly with LEG) \\
\hline & November 2007 & BRH recapitalization plan \\
\hline \multirow[t]{4}{*}{ STA } & $\begin{array}{l}\text { November } 2005 \\
\text { and May } 2006\end{array}$ & Multisector statistics \\
\hline & May, October and & Monetary and financial statistics, Government \\
\hline & $\begin{array}{l}\text { December } 2006 \text {, } \\
\text { April-May and }\end{array}$ & Finance statistics \\
\hline & November 2007 & GDDS workshop \\
\hline LEG & March 2007 & Banking law \\
\hline
\end{tabular}

\section{Resident Representative}

Mr. Ugo Fasano has been the Fund's Resident Representative since October 2005. 


\section{ANNEX II. RELATIONS With ThE INTER-AMERICAN DEVELOPMENT BANK}

(As of January 2009)

From 2003 to 2006 the Bank operationalized its development assistance to Haiti through two successive transition strategies. Currently, the Bank is operating under a new country strategy for the period 2007-11, a multiyear approach to support the consolidation of Haiti's economic and social recovery.

As of January 2009, the IDB's active portfolio has 25 investment and policy-based operations for a total of US\$712.4 million, including two investment projects receiving Board approval in December 2008. The undisbursed balance, US\$362.2 million, represents 50.8 percent of the portfolio total amount, underscoring portfolio implementation as an important challenge. In 2007, disbursements in Haiti reached US\$114.4 million, almost doubling the level registered in 2006. This positive trend was reinforced in 2008, as disbursements for 2008 totaled US\$125 million, of which US\$31.6 million were disbursed in the form of budgetary support.

The IDB has active investment projects in four key areas: a total of US\$77.5 million for state modernization and economic governance, US\$277.7 million for economic recovery, US\$109.3 million for agriculture and the environment, and US\$247.9 million for access to basic services. This package is complemented by a US $\$ 15.8$ million active portfolio in nonreimbursable technical cooperation (including Multilateral Investment Fund operations), and by non-financial products that underpin program and policy support.

In addition, the IDB manages a total of US\$75 million from other donors, including the Canadian Agency for International Development and OPEC's Fund for International Development, while an additional US\$36 million is to be approved in 2009.

In March 2007, the IDB approved debt relief for all of the Haiti's debt with the Bank accumulated prior to December 31, 2004, a total of US\$525 million. This relief will be granted when the country reaches the HIPC completion point. Interim relief of US\$10 million per year is effective for 2007 and 2008 .

As part of this debt-relief initiative, the Bank implemented the new Debt Sustainability Framework (DSF) and Performance Based Allocation System (PBA) for concessional financing, which allocated US\$50 million in grants per year to Haiti in 2007 and 2008. In addressing Government's key priorities, half of these resources (US\$50 million) have been allocated to road rehabilitation and improvement of rural and urban transport, one quarter (US\$25million) to budget support, and the remaining quarter (US\$25 million) to agricultural development and electricity infrastructure modernization.

Furthermore, interim debt relief of US\$14.15 million will be provided in 2009 to increase the Government's fiscal space to carry out vital social and economic investments and to support the country until mid-2009. 
In order to address the soaring food and energy prices earlier this year, and its negative effects on the disposable income of Haiti's poorest and most vulnerable groups, the Bank approved the transfer of US\$10 million from an existing operation to aid the Government in coping with the crisis. The operation was structured as a PBG and the Haitian Government was required to take action on several institutional and regulatory fronts to receive the grant.

In December 2008 the IDB Board of Governors approved the doubling of Haiti's grant allocation in 2009, from US\$50 million to US\$100 million. This measure was considered vital in order to help Haiti face the aftermath of 2008's devastating shocks and cover part of its financing gap, which could increase exponentially in the face of reconstruction expenses and a drying up of external financing.

The additional grants will be used in 2009 to finance reconstruction works and investment projects in key programmatic areas such as social infrastructure, urban drainage and sanitation, access to potable water in urban areas, and nutrition.

\section{Haiti's Operational Program 2009}

\begin{tabular}{|l|l|l|} 
Number & Name of the Project & $\begin{array}{l}\text { Amount (US\$ } \\
\text { million) }\end{array}$ \\
\hline HA-L1039 & $\begin{array}{l}\text { Water and Sanitation for } \\
\text { Intermediate Cities Phase II }\end{array}$ & $15 \mathrm{~m}$ \\
\hline HA-L1041 & $\begin{array}{l}\text { Natural Disaster Mitigation } \\
\text { in Priority Watersheds }\end{array}$ & $13 \mathrm{~m}$ \\
\hline HA-L1028 & $\begin{array}{l}\text { Rehabilitation of Road } \\
\text { Infrastructure III }\end{array}$ & $25 \mathrm{~m}$ \\
\hline HA-L1029 & Fiscal Consolidation I & $25 \mathrm{~m}$ \\
\hline HA-L1040 & $\begin{array}{l}\text { Support to Social } \\
\text { Infrastructure - Phase I }\end{array}$ & $20 \mathrm{~m}$ \\
\hline HA-LXXXX & Nutrition & $2 \mathrm{~m}$ \\
\hline TOTAL & & $\mathbf{1 0 0 m}$ \\
\hline
\end{tabular}

To improve program implementation, the Bank has taken specific measures, such as special procurement procedures and delegation of authority to the Country Office Representative. These measures have helped to accelerate the pace of execution, yet challenges related to the country's weak institutional capacity, small local private market, saturation of national firms and low level of foreign firm participation, remain. Along with these measures to expedite decision-making, the Bank's staff in the noted sectors has been increased at the Country Office to support the strengthening of the country's execution and absorption capacity. A financial scenario that takes into account the active portfolio, lending envelope, IDB debt 
relief, the country's absorptive capacity and the measures to accelerate execution, envisages disbursements of up to US\$150 million during the course of this year, ensuring continued positive net flows to the country.

The IDB is committed to providing comprehensive support to the country and to the priorities set for the Bank by the authorities. The Bank's programming will be reviewed and updated each year to meet the development objectives and their prioritization in the PRSP. 


\section{ANNEX III. RELATiOnS With ThE WORLd BANK GROUP}

(As of January 2009)

1. The World Bank stepped up its engagement in Haiti in March 2004, as part of a broader partnership between the Transitional Government and donors to address Haiti's social, economic and institutional needs. The government and donors conducted a needs assessment in May 2004, which provided the basis for the Interim Cooperation Framework (ICF). This established a two-year program for reform and recovery and the structures through which it would be pursued. The ICF was presented at an international donor conference in July 2004 at the World Bank headquarters, at which donor countries and international organizations pledged US\$1.1 billion for Haiti. At a subsequent conference in July 2006 in Port-au-Prince, Haiti's new elected Government extended the ICF until September 2007 and revised it to reflect Government priorities. Donors pledged US\$751 million for the period 2006-07. Additional budget support resources were mobilized during a follow-up Donors conference held in Madrid (Spain) in end-November 2006.

2. The World Bank Group's strategy and program in Haiti for FY 2007 and FY 2008 are set out in the Interim Strategy Note (ISN) reviewed by the Bank's Board on January 30, 2007. A full Country Assistance Strategy (CAS) for FY 2009-12 is being prepared and is scheduled for completion in

FY 2009 on the basis of the full Poverty Reduction Strategy Paper (Document de Stratégie Nationale pour la Croissance et la Réduction de la Pauvreté, DSNCRP) that the Government has recently completed and submitted to the World Bank and the International Monetary Fund (IMF). A Joint Staff Advisory Note (JSAN) of the DSNCRP was discussed by the Boards of the IMF and the World Bank in January and March 2008, respectively. The most recent full CAS was discussed by the Board in 1996.

3. Since Haiti's arrears were cleared in January 2005, IDA has approved 14 projects for US\$212 million. These comprise: two development policy operations and two technical assistance grants in support of economic governance reform; 9 investment projects (for disaster response and management, community-driven development, transport and territorial development, electricity, rural water and sanitation; and education); and a catastrophe risk insurance grant. Since FY 2006 all assistance has been in grant form. All projects but one remain active. In addition, more than US\$13 million of trust fund grants, mostly from the LICUS and Post-Conflict trust funds have been awarded, since 2004.

4. In FY 2008, in the context of a declining IDA envelope, the Bank has narrowed its (non-emergency) assistance to three main areas in Haiti: education, community-driven development and economic governance. The Bank expects this focus to continue, but will determine the scope of future assistance in discussions with authorities and other stakeholders through the preparation of a new CAS for FY 2009-12. The strategy will align Bank assistance behind Haiti's PRSP. The country has tapped the maximum of three 
exceptional allocations allowed for re-engaging countries under IDA 14, having received US\$75 million in FY 2005, US\$63 million in FY 2006 and US\$62 million in FY 2007. In FY 2008, the total has dropped to around US\$41 million, including US\$17 million of additional emergency assistance (with a post-Tropical Strom Noel grant of US\$7.4 million). Under new IDA rules the country will qualify for two more years of special allocations (FY 2009 and FY 2010), but this will be a reduced, phased-out amount.

5. In addition, the Bank has completed seven major analytical works, including a Country Economic Memorandum (CEM), a Country Social Analysis, and a Social Protection Strategy, and a joint World Bank-IADB Public Expenditure Management and Financial Accountability Review (PEMFAR). The PEMFAR provides an analytical basis in support of the Government's medium and longer term public finance reform program. The PEMFAR examines the linkages between public finance, growth and poverty with a view to helping policymakers in Haiti design the new generation of public finance reforms centered on policy actions to promote sustained and equitable growth and reduce poverty. Following the PEMFAR findings and policy recommendations, the Government prepared in November 2007 its action plan, which includes priorities to advancing public finance reforms in the short and medium terms. The action plan will be a unique policy matrix on which donors will base their support to the Government's public finance reforms in the next three to five years. The Bank has also undertaken a Financial Sector Assessment Program (FSAP) jointly with the IMF in FY 2008.

6. An Interim Poverty Reduction Strategy Paper was prepared by the Government and presented to the IMF and World Bank Boards, together with the Joint Staff Advisory Note (JSAN), in November 2006 at the time of discussion of the HIPC Decision Point Document. A Preliminary HIPC Document, prepared jointly by the Bank and IMF staff in collaboration with the Government, was discussed by the Boards of the IMF and the Bank in September 2006. The final HIPC Decision Point document was discussed by both boards in November 2006. Haiti is scheduled to reach the completion point under the Enhanced HIPC framework in early 2009. World Bank and IMF staffs are closely monitoring progress in implementing the HIPC triggers. Bank and Fund staffs are also monitoring closely Haiti's debt situation. In this regard, the staffs of the two institutions prepared a new Debt Sustainability Analysis in February 2008.

7. The International Finance Corporation (IFC) is working to identify specific actions to promote a sound business-enabling environment, while investing in projects that support the development of a sustainable private sector and income-generating activities. Recent investments have been in cellular telecommunications (Digicel-US\$15 million in each of FY 2006 and FY 2007), textiles (Grupo M-US\$20 million in FY 2004), and microfinance (MicroCredit National-US\$0.4 million in FY 2004). In May 2008, the IFC approved a US\$2.0 million trade finance line for Capital Bank. In addition, IFC is discussing with the Government the provision of advisory support in the design and implementation of private 
sector participation transactions for the airport of Port-au-Prince and for state-owned telecom (TELECO). Additional support is envisaged through the IFC LAC Facility and the Foreign Investment Advisory Service (FIAS), possibly in the following areas: (1) business simplification; (2) improving access to finance; (3) investment facilitation (notably textiles); and (4) training, through the SME Toolkit and Business Edge. 
Press Release No. 09/34

International Monetary Fund

FOR IMMEDIATE RELEASE

Washington, D.C. 20431 USA

February 13, 2009

\section{IMF Executive Board Completes Fourth Review under PRGF Arrangement with Haiti and Approves US\$36.6 Million Augmentation and US\$35.8 Million Disbursement}

The Executive Board of the International Monetary Fund (IMF) has completed the fourth review of Haiti's economic performance under the Poverty Reduction and Growth Facility, and approved an increase in financial assistance of an amount equivalent to SDR 24.57 million (about US\$36.6 million) to mitigate the negative effects caused by a series of hurricanes in 2008 as well as the global downturn. The completion of the review enables Haiti to receive an immediate disbursement of an amount equivalent to SDR 23.98 million (about US\$35.8 million), bringing total disbursements to SDR 91.3 million (about US\$136.1 million). The Executive Board also granted a waiver for the non-observance of a September 2008 quantitative performance criterion related to a minor deviation of net central bank credit to the nonfinancial public sector.

The three-year PRGF arrangement was approved in November 2006 in an original amount of SDR 73.71 million (about US\$109.9 million) (see Press Release No. 06/258). At the same time, the IMF and the World Bank determined that Haiti qualified for debt relief under the enhanced Heavily Indebted Poor Countries Initiative (HIPC) (see Press Release No. 06/261). In June 2008, the Executive Board approved the first augmentation under the PRGF arrangement in an amount equivalent to SDR 16.38 million (about US\$24.4 million; see Press Release No. 08/145) to help Haiti cope with the impact of high international food and fuel prices.

Following the Executive Board discussion, Mr Takatoshi Kato, Deputy Managing Director and Acting Chair, issued the following statement:

"The Haitian authorities are to be commended for maintaining macroeconomic stability and advancing with structural reforms during 2008, in spite of a succession of severe shocks to the economy. Higher food and fuel prices and resulting political disturbances severely constrained government operations, while a series of hurricanes and tropical storms caused unprecedented economic losses. 
"The authorities remain firmly committed to their economic program, which seeks to strike a balance between safeguarding macroeconomic stability and pursuing economic and social development. In view of the large spending needs related to infrastructure reconstruction and poverty-reduction priorities, further efforts to raise domestic revenue are needed. However, the support of the international community will be crucial, and the authorities are encouraged to continue to work closely with donors to mobilize additional aid.

"Provided remaining HIPC completion point triggers are implemented in a timely manner, Haiti should benefit from HIPC/MDRI debt relief by mid-2009. Going forward, maintaining debt sustainability will hinge on a cautious approach to new borrowing and the implementation of policies to promote economic growth and export diversification.

"Haiti will continue to face difficult challenges in the period ahead. The political and social situation remains fragile, the impact of weather-related shocks lingers on, and the global downturn is expected to negatively affect remittances and exports. The authorities' impressive performance and firm commitment to the program provide a strong basis for support from the Fund and the international community. Timely and adequate donor support will be crucial to preserve Haiti's hard-won gains of recent years, implement its poverty reduction strategy, and boost economic growth,” Mr. Kato said.

The PRGF is the IMF's concessional facility for low-income countries. PRGF-supported programs are based on country-owned poverty reduction strategies adopted in a participatory process involving civil society and development partners and articulated in the country's Poverty Reduction Strategy Paper. This is intended to ensure that PRGF-supported programs are consistent with a comprehensive framework for macroeconomic, structural, and social policies to foster growth and reduce poverty. PRGF loans carry an annual interest rate of 0.5 percent and are repayable over 10 years with a $5 \frac{1}{2}$-year grace period on principal payments. 


\section{Statement by Nogueira Batista, Executive Director for Haiti and Ketleen Florestal, Advisor to the Executive Director for Haiti}

February 11, 2009

The setting within which the PRGF program was implemented during the period under review was exceptionally difficult. The second half of FY08 was a period of protracted political stalemate as it took four months and three nominees for the Parliament to approve the Prime Minister who was to succeed PM Alexis, whose dismissal in April 2008 was set off by the food and oil price crisis. Within the period under review, four tropical storms and hurricanes have caused considerable damage to Haiti's infrastructure and agriculture and inflicted increased hardship on the population. In addition, the global financial crisis is likely to impact negatively on the flow of remittances (about 25\% of GDP) and exports. Despite these turbulences and severe external shocks, the Haitian authorities have managed to maintain macroeconomic stability and move forward with the structural reform agenda, including significant progress in the realization of completion point triggers.

This fourth review of the PRGF represents an opportunity to take stock of Haiti's strong performance under the program and to restate our concerns about the adequacy of the IMF's response. It is disconcerting that, in spite of Haiti's track record, it took close to four months of negotiations to bring to the Executive Board the request of access augmentation under the PRGF. Moreover, despite extremely difficult economic, social and political circumstances, repeated external shocks and important balance of payment needs, the augmentation was capped at the present ceiling for normal access under the PRGF (140 percent) and is being disbursed in two tranches.

The authorities are strongly committed to respecting the engagements taken for this third year of the program. However, the austerity of the program and the challenges or opposition it may face in its implementation need to be underscored. The program for FY09 leaves very little room to address the urgent post-hurricane reconstruction and humanitarian needs and has the potential to stifle future growth. The Fund should make good on its promise to show flexibility if the impact of the global crisis worsens or other external shocks make it necessary to recalibrate the parameters of the program.

The IMF's traditional catalytic and signaling role also needs to be revisited. The staff report repeatedly and rightly underscores the tininess of donor support relative to Haiti's increased needs following the serious infrastructure and crop destruction caused by natural disasters . Even though the government has scaled back considerably reconstruction and development investments, the financial gap remains substantial for FY09 (US\$ 50 million) and the financing of the PRSP continues to be uncertain. The United Nation's humanitarian flash appeal -- after back-to-back storms and hurricanes that had destroyed infrastructure and crops equivalent to about 15 percent of GDP -- led to the pledging and disbursement of the equivalent of only a little over 1 percent of GDP. Sustained donor support is crucial for maintaining economic, social and political stability, which has been achieved with large- 
scale financial and technical assistance coupled with strong international political support. We encourage the Fund to strengthen its efforts to disseminate information on Haiti's impressive performance under the PRGF program and to help secure additional financial assistance. The success of the upcoming donors' conferences in March and April 2009 will need to be measured not only by the level of new pledges but also by donors' willingness to realign their programs to the nationally defined priorities and to design efficient aid delivery strategies.

The Haitian authorities are thankful to Haiti's friends who have scaled up their financial and technical support in response to recent disasters. They are particularly appreciative of the IDB's efforts to double its grant allocation for FY09. We urge bilateral donors as well as regional and multilateral donors (World Bank, IDB, EU) to revisit their traditional allocation benchmarks, to find innovative ways to increase their support to Haiti, and to adapt their aid programs to the new circumstances and the government's strategy. We encourage the Fund's management and our colleagues in the Board to stress to donors the need for an increased share of budget support in order to close the program's financial gap. At the same time, donors should be urged to direct investment funding towards priority sectors defined in the PRSP, which the government is revising in light of the recent shocks. Any reluctance to work within the PRSP framework would not only be costly to Haiti, but could also be considered a setback to the IMF's (and the World Bank's) credibility as the PRSP approach has been adopted by Haiti with the support of the Bretton Woods institutions.

At the time of the third review of the PRGF, some Directors expressed concerns about the potential risk of Petrocaribe financing to debt sustainability, although these loans are highly concessional. The Haitian authorities are committed to the prudent management of external debt, but they wish to ensure that sound growth and poverty reduction opportunities are not needlessly forgone. It would not therefore be advisable to completely exclude debt contracting, especially when loans are highly concessional. Notwithstanding the important external shocks that hit the economy during the past year and Haiti's strong track record, assistance in the form of grants has not been sufficiently forthcoming. During the period under review, Petrocaribe's funds have not only allowed the observance of performance criteria, as staff observes, but have also been the main source of financing of the government's emergency program to respond to the population's immediate needs after the natural disasters. It would be important in future Debt Sustainability Analyses (DSAs) to take into account not only the amounts of debt contracted but also the quality of the investments these loans help finance, particularly in terms of offsetting some of the negative impact of shocks.

The challenges ahead are numerous and the downside risks to the program are indeed abundant. They stem chiefly from the indefinite length and severity of the global crisis and the unknown outcome of the upcoming donors' conferences. The impact of the current decline in fuel prices on the flow of resources available through Petrocaribe is also a concern. On the upside, the absorptive capacity has been significantly increased and respectable 
growth levels can be expected if investment funds are made available and the HOPE initiative is fully exploited.

All completion point triggers are projected to be achieved by June 2009, with the exception of the one relating to the procurement law. Postponing the delivery of full debt relief, even if only by two months, will be very costly to Haiti. The delay in the submission of the procurement law to Parliament is mainly due to the political stalemate of 2008, and the authorities expect the draft law to be voted before the end of February. We call on the Boards of the World Bank and the IMF to be flexible on the length of implementation of the procurement law necessary to reach the completion point. It is important to recall that the procurement legislation has undergone significant transformations since 2004. The changes included in the draft legislation pending approval by Parliament are additional improvements the authorities are committed to implement in order to achieve international standards. 